United States Department of Commerce Technology Administration

National Institute of Standards and Technology

NISTIR 3993

\title{
A BIBLIOGRAPHY OF THE NIST ELECTROMAGNETIC FIELDS DIVISION PUBLICATIONS
}

Edited by

Ruth Marie Lyons

Kathryn A. Gibson 



\section{A BIBLIOGRAPHY OF THE NIST \\ ELECTROMAGNETIC FIELDS \\ DIVISION PUBLICATIONS}

\section{Edited by}

Ruth Marie Lyons

Kathryn A. Gibson

Electromagnetic Fields Division

Electronics and Electrical Engineering Laboratory

National Institute of Standards and Technology

Boulder, Colorado 80303-3328

\section{August 1992}

(Supersedes NISTIR 3973)

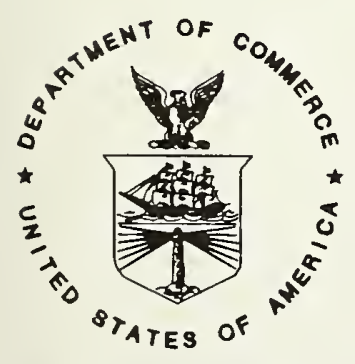

U.S. DEPARTMENT OF COMMERCE, Barbara Hackman Franklin, Secretary TECHNOLOGY ADMINISTRATION, Robert M. White, Under Secretary for Technology NATIONAL INSTITUTE OF STANDARDS AND TECHNOLOGY, John W. Lyons, Director 
CONTENTS

$\underline{\text { Page }}$

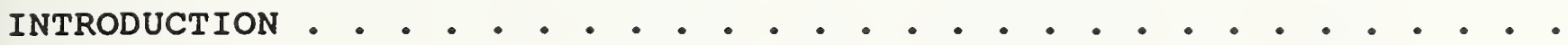

ANTENNAS

DIELECTRIC MEASUREMENTS • • • • • • • • • • • • • • • • • • • • • • • •

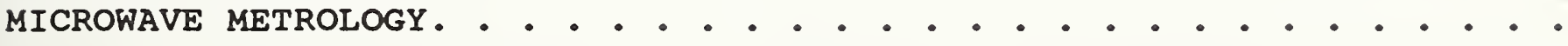

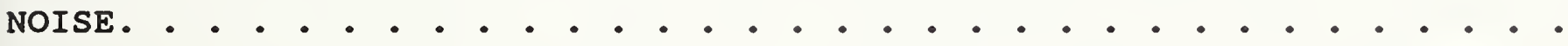

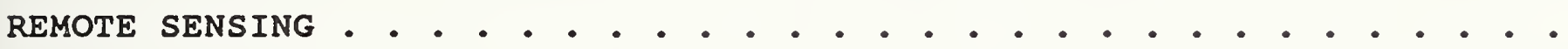

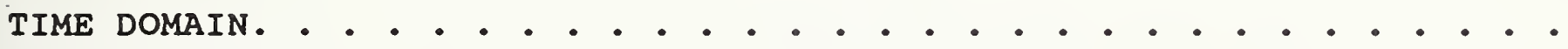

80

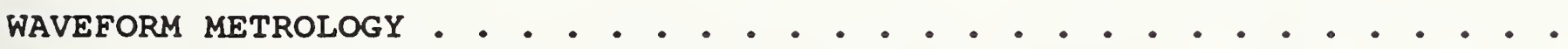

MISCELLANEOUS • • • • • • • • • • • • • • • • • . • • • • • . . . • • 
, 


\title{
A BIBLIOGRAPHY OF THE NIST ELECTROMAGNETIC FIELDS DIVISION PUBLICATIONS
}

\author{
Edited by
}

Ruth Marie Lyons and Kathryn A. Gibson

This bibliography lists the publications by the staff of the Electromagnetic Fields Division of the National Institute of standards and Technology for the period January 1970 through July 1992. It supersedes NISTIR 3973 which listed the publications of the Electromagnetic Fields Division from January 1970 through July 1991. Selected earlier publications from the Division's predecessor organizations are included.

Key words: antennas; dielectric measurements; electromagnetic interference; microwave metrology; microwave power, impedance, attenuation; near-field antenna measurements; noise; nonionizing radiation; radiation hazards; standards; time domain; waveform metrology.

\section{INTRODUCTION}

The Electromagnetic Fields Division was formed during the reorganization of NIST (formerly NBS) in April 1978. Originally, the Division developed measurement methods and standards, and provided metrological support for antenna systems, noise measurement equipment, fast pulse waveforms, electromagnetic interference, electromagnetic environmental characterization equipment, electromagnetic emission and immunity (or susceptibility) testing equipment, and especially for fields near sources or in other complicated field conditions. In April 1985, microwave and millimeter wave metrology was added to the Division.

The editors have attempted to include all work published by the Division staff in the period from January 1970 through July 1992. Work performed by authors before joining this Division or work by authors who have left this Division has been included only when it had special significance. A miscellaneous category includes general

publications. A few references are listed in more than one category where appropriate.

Several other information sources may be useful to the reader who is interested in NIsT activities connected with electromagnetic metrology. A companion bibliography, NIsTIR 3994, lists publications of the Electromagnetic Technology Division for the same period. Topics include metrology for laser systems, optical fibers, optical communication equipment, cryoelectronics, superconductors, and other unusual electrical 
engineering materials. Three additional bibliographies list publications of the form Electromagnetics Division: NBSIR 73-320 (July 1972-June 1973); NBSIR 74-395 (July 1973-June 1974); NBSIR 75-818 (July 1974-June 1975). These were preceded by a series of unpublished reports (edited by H. M. Altschuler) that cover the period back to 195 An excellent summary of the whole field of electromagnetic metrology as it stood in 1967 was published as a special issue of the IEEE Proceedings (volume 55, June 1967). Advances in the following decades were described in two other special issues of the same journal (volume 66, April 1978 and volume 74, January 1986).

\section{A Note on Abbreviations}

NOTE: On August 23, 1988, the National Bureau of Standards (NBS) became the National Institute of Standards and Technology (NIST); therefore, documents with either prefix are considered NIST publications. Most readers are familiar with the commonly used abbreviations for the names of the professional journals that appear in this bibliography. Some publication series are peculiar to NIST and may call for explanation. They are:

NISTIR - NIST Interagency/Internal Report NIST Tech. Note - NIST Technical Note

NIST SP - NIST Special Publication

NIST HB - NIST Handbook

NIST JRES - NIST Journal of Research

NIST MN - NIST Monograph
NBSIR - NBS Interagency/Internal Report NBS Tech. Note - NBS Technical Note NBS SP - NBS Special Publication NBS HB - NBS Handbook

NBS JRES - NBS Journal of Research NBS MN - NBS Monograph

Purchase Procedures and Document Availability

NIST (NBS) Technical Notes, Special Publications, Handbooks, Journals of Research, and Monographs may be purchased from the superintendent of Documents, U. S. Government Printing office, Washington, DC 20402. Orders must be accompanied by postal money order, express money order, or check made out to the superintendent of Documents.

NIST (NBS) Interagency/Internal Reports (NISTIRs, NBSIRs) may be purchased from the National Technical Information Service, Springfield, VA 22161. Orders must be accompanied by postal money order, express money order, or check made out to NTIS.

Reprints of papers published in non-NIST media may be available in limited quantities from the authors. 
BAIRD, R. C.

Microwave Antenna Measurement Services at the National Bureau of

Standards

Dig., Antenna Meas. Tech. Assoc. Mtg.

October 1981

BAIRD, R. C.; ESTIN, A. J.

The Orbiting Standards Package: A Recalibratable satellite Instrument

Assembly for Measuring Large Earth Station Antennas

Proc., 1982 Antenna Meas. Tech. Assoc., 5-7 October, 1982, New

Mexico State University, Las Cruces, NM, pp. 5-1 through 5-9 1982

BAIRD, R. C.; RERNS, D. M.

The Accurate Determination of Millimeter-Wave Antenna Characteristics by Deconvolution and Extrapolation Techniques

Proc., 1974 Millimeter-Wave Conf., 26-28 March, 1974, Vol. 2,

E 2-1 through E 2-12

1974

BAIRD, R. C.; NEWELL, A. C.; STUBENRAUCH, C. F.

A Brief History of Near-Field Measurements of Antennas at the National

Bureau of Standards

IEEE Trans. Antennas Propagat., Spec. Issue on Near-Field Scanning Techn., Vol. 36, No. 6, pp. 727-733 June 1988

BAIRD, R. C.; DAYWITT, W. C.; NEWELL, A. C.; PERERA, S.; REPJAR, A. G.; WAIT, D. F.; ESTIN, A. J.

Calibration Requirements for EHF satellite Communication systems

NBSIR 86-3058

October 1986

BENSEMA, W. D.

Broadband Orthogonal Array Antenna System: Microprocessor Control and Computation

Record, "EMC '81," 1981 IEEE Intl. Symp. on Electromagn. Compat., 18-20 August 1981, Boulder, CO 1981

Handbook for Broadband Isotropic Antenna System Volume 1 - Operations Manual

$$
\begin{array}{ll}
\text { NBSIR } & 83-1693 \\
\text { July } 1983
\end{array}
$$

BUSSEY, H. E.; LARSEN, E. B .

Buried Antenna Performance: Development of Small Resonant Buried Antennas

$$
\begin{gathered}
\text { RADC-TR-74-169M, AD } 783274 \\
\text { June } 1974
\end{gathered}
$$

CRAWFORD, M. L.

Calibration of Broadbeam Antennas Using Planar Near-Field Measurements Proc., 1976 Conf. Prec. Electromagn. Meas., 28 June-1 July 1976, Boulder, CO, pp. 53-56

$$
1976
$$

CRUZ, J. E.; LARSEN, E. B.

Screenroom Measurements of Antenna Factors

Proc., 1985 Instrum. and Meas. Technol. Conf., 20-22 March 1985, Tampa, FL, p. 208 1985 
DAY, G. W.; STUBENRAUCH, C. F.

Laser Far-Field Beam-Profile Measurements by the Focal Plane Technique NBS Tech. Note 1001 March 1978

DAYWITT, W. C.

Error Equations Used in the NBS Precision G/T Measurement system NBSIR 76-842

september 1976

A Precision Earth-Terminal system for Accurate C/KT, G/T, and EIRP Measurements with a Calibrated Radio star Proc., 1977 Intl. Union of Radio Sci. (URSI) Symp. on Meas. in Telecommunications, 3-7 October 1977, Lannion, France, pp. 1-4 1977

Error Equations Used in the NBS Earth-Terminal Measurement system NBSIR 78-869

December 1977

Atmospheric Propagation Equations Used in the NBS Earth Terminal

Measurement system

NBSIR 78-883

April 1978

An Error Analysis for the Measurement of Satellite EIRP using a Calibrated Radio star

IEEE Trans. Instrum. Meas., Vol. IM-27, No. 3, pp. 253-258 September 1978

An Error Analysis for the Use of Presently Available Lunar Radio Flux Data in Broadbeam Antenna-System Measurements NBS Tech. Note 1973 February 1984

A Preliminary Investigation into Using the Sun as a source for G/T Measurements

NBSIR 84-3015

August 1984

A Simple Technique for Determining Joint Losses on a Coaxial Line from Swept-Frequency Reflection Data

IEEE Trans. Instrum. Meas., Vol. IM-36, pp. 468-473 June 1987

DAYWITT, W. C.; RANDA, M.

G/T Measurement Errors with Radio stars Proc., 1975 IEEE Intl. Antennas and Propagat. Symp., 2-4 June 1975, Urbana-Champaign, IL, Session 20, pp. 460-463 1975

DOUGHERTY, H. T.; ESTIN, A. J.; MORGAN, W. L.; WOODRUFF, J. J. The Orbiting standards Platform

Proc., 1978 Antenna Applications Symp., 20-22 september 1978, Urbana-Champaign, IL, pp. 1-9 1978

ESTIN, A. J.; BAIRD, R. C.

Feasibility study of Orbiting standards Platform NBSIR 78-886

June 1978 
ESTIN, A. J.; JANEZIC, M. D.

Improvements in Dielectric Measurements with a Resonant Cavity

Conf. Record, IEEE Instrum. Meas. Tech. Conf., 14-16 May 1991,

Atlanta, GA, pp. 573-579

May 1991

ESTIN, A. J.; STUBENRAUCH, C. F.; REPJAR, A. G ; NEWELI, A. C.

Optimized Wavelength-Sized Scalar Horns as Antenna Radiation standards

1981 EEMTIC Dig., June 1981 1981

IEEE Trans. Instrum. Meas., Vol. IM-31, No. 1, pp. 53-56 March 1982

FITZGERRELL, R. G.

A Partial Loop Source of $\mathbf{E} \& \mathrm{H}$ Fields for Antenna Factor Calibration (A Loop Cell)

Proc., 1982 Ant. Meas. Tech. Assoc., 5-7 October 1982, New Mexico State University, Las Cruces, NM, pp. 15-1 through 15-22 1982

A Source of E\&H Fields for Antenna-Factor Calibration

IEEE Trans. Electromagn. Compat., Vol. EMC-26, No. 2, pp. 58-65 May 1984

Linear Gain - Standard Antennas Below $1000 \mathrm{MHz}$ NBS Tech. Note 1098

May 1986

Standard Linear Antennas, $30 \mathrm{GHz}$ to $1000 \mathrm{MHz}$

Proc., Conf. on Electromagn. Compat., 29 september-2 October 1986, University of York, England, pp. 147-153 1986

Three PC-Computer Programs for Antenna Calculations Primarily for Use Below $1000 \mathrm{MHz}$

IEEE Ant. Propagat. Soc. (AP-S) Newslett., Vol. 29, No. 3, pp. $30-32$

June 1987

FRANCIS, M. H.

Antenna Far-Field Pattern Accuracies at Millimeter Wave Frequencies

Using the Planar Near-Field Technique.

Proc., Ant. Meas. Tech. Assoc. Symp., 9-13 October 1989, Monterey, CA, pp. 11-16 through 11-21

October 1989

FRANCIS, M. H.; HILI, D. A.

Out-of-Band Response of Array Antennas

Proc., Ant. Meas. Tech. Assoc., 28 September-2 October 1987,

Seattle, WA, pp. 14-19 1987

FRANCIS, M. H.; MACREYNOLDS, K.

Evaluation of Dual-Port Circularly Polarized Probes for Planar Near-

Field Measurements

Proc., 12th Annual Meeting \& Symp., Ant. Meas. Tech. Assoc., 8-11

oct. 1990, Philadelphia, PA, pp. 13-3 through 13-8

october 1990 
FRANCIS, M. H.; STUBENRAUCH, C. F.

Comparison of Measured and Calculated Antenna Side Lobe Coupling Loss in

the Near Field Using Approximate Far-Field Data

IEEE Trans. Antennas Propagat., Vol. 36, No. 3, pp. 438-441 March 1988

FRANCIS, M. H.; WITTMANN, R. C.

Swept Frequency Gain Measurements from 33-50 GHz at the National

Institute of Standards and Technology

Proc., 13th Annual Meeting \& Symp., Ant. Meas. Tech. Assoc., 7-11

Oct. 1991, Boulder, CO, pp. 1-3 through 1-6 October 1991

FRANCIS, M. H.: YAGHJIAN, A. D.

Computations of Antenna Side-Lobe Coupling in the Near Field Using

Approximate Far-Field Data

NBSIR 82-1674

August 1982

FRANCIS, M. B.; KREMER, D. P.; REPJAR, A. G.

Advanced system Characterizes Antennas to $65 \mathrm{GHz}$

Microwaves and RF pp. 77-88

March 1990

FRANCIS, M. H.; REPJAR, A. G.; RREMER, D. P.

Antenna Measurements for Millimeter Waves at the National Bureau of Standards

Proc., Ant. Meas. Tech. Assoc., 12-16 september 1988, Atlanta, GA, pp. 13-13 through 13-17

september 1988

A Certification Plan for a Planar Near-Field Range Used for HighPerformance Phased-Array Testing

NISTIR 3991

July 1992

GREENLEE, D. H.; RANDA, M.; CHANG, D. C.

The Characteristics of Iris-Fed Millimeter Wave Rectangular Microstrip

Patch Antennas

NBS Tech. Note 1063

October 1983

GUERRIERI, J.R.; RREMER, D. P .

Automated Multi-Axis Motor Controller and Data Acquisition system for Near-Field Scanners.

Proc., Ant. Meas. Tech. Assoc. Symp., 9-13 October 1989, Monterey, CA, pp.12-24 through 12-28

october 1989

HILU, D. A.

Theory of Near-Field Phased Arrays for Electromagnetic susceptibility Testing

NBS Tech. Note 1072

February 1984

Out-of-Band Response of Reflector Antennas

NBSIR 85-3021

April 1985

Far-Field Transient Response of an Antenna from Near-Field Data NBSIR 86-3063

December 1986 
BIIL, D. A. (cont.)

Antennas for Geophysical Applications Antenna Handbook: Theory, Applications and Design (Chapter 3), edited by U. T. Lo and S. W. Lee, Van Nostrand Reinhold Co., pp. 23-1 through 23-6 1988

HILI, D. A.; CAVCEY, K. B.

Coupling Between Two Antennas separated by a Planar Interface

IEEE Trans. Geosci. Remote Sensing, Vol. GE-25, No. 4, pp. 422-431 July 1987

BII工, D. A.; EBRET, R. L.

Near-Field Gain of Pyramidal Horns from 18 to $40 \mathrm{GHz}$

NISTIR 89-3924

November 1989

HILL, D. A.; FRANCIS, M. B.

Out-of-Band Response of Antenna Arrays

NBSIR 86-3047

June 1986

Proc., 1987 IEEE Intl. Symp. Electromagn. Compat.,

25-27 August 1987, Atlanta, GA, pp. 435-438 1987

IEEE Trans. Electromagn. Compat., Vol. EMC-29, No. 4, pp. $282-288$

November 1987

BILI, D. A.; ROEPKE, G. H.

A Near-Field Array of Yagi-Uda Antennas for Electromagnetic

Susceptibility Testing

NBS Tech. Note 1082

July 1985

IEEE Trans. Electromagn. Compat., Vol. EMC-28, No. 4, pp. 273-276

November 1986

An Array of Dipoles for Plane Wave Synthesis

Proc., 1985 Intl. Symp. On Antennas and EM Theory, 26-28 August

1985, Beijing, China, pp. 422-427 1985

BILI, D. A.; WAIT, J.R.

Electromagnetic Characteristics of a Coaxial Cable with Periodic slots

IEEE Trans. Electromagn. Compat., Vol. EMC-22, No. 4,

pp. 303-307

November 1980

JESCB, R. I .

Measured Vehicular Antenna Performance

IEEE Trans. Veh. Technol., Vol. VT-34, No. 2, pp. 97-107 May 1985

National Institute of Justice Technology Program, NIJ Report-201-85

May 1986 
KANDA, M.

Accuracy Considerations in the Measurement of the Power Gain of a Large Microwave Antenna

Proc., 1974 Intl. Ant. Propagat. Soc. Symp., 10-12 June 1974, Atlanta, GA, pp. 43-45 1974

Accuracy Considerations in the Measurement of the Power Gain of a Large Microwave Antenna

IEEE Trans. Antennas Propagat., Vol. AP-23, No. 3, pp. 407-411

May 1975

Study of Error Analysis for Absolute Flux Density Measurements of Cassiopeia

IEEE Trans. Instrum. Meas., Vol. IM-25, No. 3, pp. 173-182

september 1976

A Relatively Short Cylindrical Broadband Antenna with Tapered Resistive Loading for Picosecond Pulse Measurements

NBSIR 77-861

August 1977

IEEE Antennas Propagat. Soc. Dig., pp. 230-233

May 1978

IEEE Trans. Antennas Propagat., Vol. AP-26, No. 3, pp. 439-447

May 1978

Transients in Resistively Loaded Antennas and Their Comparison with Conical Antennas and TEM Horns

NBSIR 78-876

March 1978

IEEE Trans. Antennas Propagat., Vol, AP-28, No. 1, pp. 132-136

January 1980

Analytical and Numerical Techniques for Analyzing an Electrically Short Dipole with a Nonlinear Load

NBSIR 78-898

November 1978

IEEE Trans. Antennas Propagat., Vol. AP-28, No. 1, pp. 71-78 January 1980

The Time Domain Characteristics of a Traveling Wave Linear Antenna with Linear and Non-Linear Loads

NBSIR 78-892(R)

February 1979

The Effects of Resistive Loading on TEM Horns

NBSIR 79-1601

August 1979

The Characteristics of a Linear Antenna with Tapered Resistive and Capacitive Loading

Proc., 1980 IEEE Ant. Propagat. Soc. Intl. Symp., 2-6 June 1980, Quebec, Canada, Vol. 2, AP. 18-2, pp. 696-699 1980 
RANDA, M. (cont.)

The Effects of Resistive Loading on TEM Horns

IEEE Trans. Electromagn. Compat., Vol. EMC-24, No. 2, pp. 245-255

May 1982

An Electromagnetic Near-Field sensor for simultaneous Electric and Magnetic-Field Measurements

IEEE Trans. Electromagn. Compat., Vol. EMC-26, No. 3, pp. 102-110

August 1984

Transients in a Resistively Loaded Loop Antenna

Proc., 1984 Intl. Symp. on Electromagn. Compat., 16-18 October

1984, Tokyo, Japan, Vol. 1, pp. 286-290 1984

KANDA, M.; ORR, R. D.

Near-Field Gain of a Horn and an Open-Ended Waveguide; Comparison

Between Theory and Experiment

IEEE Ant. Propagat Soc. Symp. on Antennas and Propagation, 8-13

June 1986, Philadelphia, PA, pp. 91-94 1986

Proc., 5th Intl. Conf. on Electromagn. Compat., 29 september-

2 October 1986, University of York, England, pp. 137-145 1986

IEEE Trans. Antennas Propagat., Vol. AP-35, No. 1, pp. $33-40$

January 1987

RANDA, M.; CEANG, D. C.; GREENLEE, D. B.

The Characteristics of Iris-Fed Millimeter Wave Rectangular Microstrip Patch Antennas

Proc., 1982 IEEE Ant. Propagat. Soc. Symp., 24-28 May 1982,

University of New Mexico, Albuquerque, NM, pp. 292-295 1982

RERNS, D. M.

Comment on Correction of Errors in Aerial Far-Field Radiation-Pattern Determinations

Electronics Lett., Vol. 7, No. 24, pp. 706

December 1971

Plane-Wave Scattering Matrix Theory of Antennas and Antenna-Antenna

Interactions: Formulation and Applications

NBSIR 75-824

January 1975

Plane-Wave Scattering Matrix Theory of Antennas and Antenna-Antenna

Interactions

NBS MN 162

June 1981

RREMER, D. P.; NEWEIL, A. C.

Alignment Fixture for Millimeter Waveguide

IEEE Antennas \& Propagation Magazine, Vol. 32, No. 3, pp. 45-48 June 1990 
KREMER, D. P.; REPJAR, A. G.

Antenna Calibrating Standards Using $\mathrm{CW}$ and Pulsed-CW Measurements and the Planar Near-Field Method

Proc., Ant. Meas. Tech. Assoc., 12-16 september 1988, Atlanta, GA, pp. 13-21 through 13-29

September 1988

KREMER, D. P.; NEWELT, A. C.; AGEE, D. A.

Absorber Characterization.

Proc., Ant. Meas. Tech. Assoc. Symp. 9-13 October 1989, Monterey, $\mathrm{CA}, \mathrm{pp} \cdot 13-7$ through 13-11. October 1989

LARSEN, E. B.

Calibration and Meaning of Antenna Factor and Gain for EMI Antennas Interference Technology Engineers' Master (ITEM 1986), pp. $113-335$

1986

LARSEN, E. B .; ANDREWS, J. R.

Using Fiber Optics in a Broadband, Sensitive, Isotropic Antenna $15 \mathrm{kHz}$ to $150 \mathrm{MHz}$

Proc., IEEE Intl. Symp. on Electromagn. Compat., 13-15 July 1976, Washington, DC, pp. 385-389 1976

LARSEN, E. B.; CRUZ, J. E.

E\&H Fields in Transmission Lines and Coils for susceptibility Testing, Probe Calibration, and RF Exposure Chambers

Proc., 1985 Instrum. and Meas. Technol. Conf., 20-22 March 1985, Tampa, FL, p. 199 1985

LAWTON, R. A.; ONDREJKA, A. R.

Antennas and the Associated Time Domain Range for the Measurement of Impulsive Fields

NBS Tech. Note 1008 November 1978

LEWIS, R. I.

The Use of Three Term Recursion Relations for Numerical Computations as Applied to Near-Field Spherical Scanning

Proc., Intl. Union of Radio Science (URSI) Intl. Symp., 20-24 June 1977 , pp. 224-226 1977

Efficient Computation of the Far-Field Radiated by an Arbitrary Rectangular-Aperture Distribution NBSIR 81-1643

April 1981

Spherical Scanning Data Processing: An Algorithm for Halving the Data Processing Effort when the Radiation into the Back Hemisphere is Negligible

Dig., 1981 IEEE Ant. Propagat. Soc. Intl. Symp. June 1981

Spherical-Wave Source-Scattering-Matrix Analysis of the Mutual Coupling Between Two Antennas

Dig., 1981 IEEE Ant. Propagat. Soc. Intl. Symp. June 1981 
IEWIS, R. L. (cont.)

Spherical-Wave Source-Scattering Matrix Analysis of Coupled Antennas; A General system Two-Port Solution

IEEE Trans. Antennas Propagat., Vol. AP-35, No. 12 ,

pp. 1375-1380

December 1987

LEWIS, R. L.; NEWELL, A. C.

Efficient and Accurate Method for Calculating and Representing Power Density in the Near Zone of Microwave Antennas

IEEE Trans. Antennas Propagat., Spec. Issue, Near-Field Scanning

Techn., Vol. 36, No. 6, pp. 890-891

June 1988

LEWIS, R. L.; WITTMANN, R. C.

Improved Spherical and Hemispherical scanning Algorithms

IEEE Trans. Antennas Propagat., Vol. AP-35, No. 12 ,

pp. 1381-1388

December 1987

MA, M. T.

A Theoretical Study of Unbalanced Ground Effects on Receiving Dipoles NBSIR 79-1605

May 1979

Synthesis of Broadband Antenna Arrays as Possible Over-the-Horizon Radars Research Topics in EM Wave Theory (Chapter 9)

John Wiley Interscience, pp. 188-209

1981

Arrays of Discrete Elements

Antenna Engineering Handbook, Chapter 3, R. C. Johnson and H.

Jasik, McGraw-Hill Book Co.

January 1984

MUTH, L. A.

Mutual Impedances and Multiple Reflections in an N-Element Array

Environment

NBS Tech. Note 1078

February 1985

A Theory of Mutual Impedances and Multiple Reflections in an N-Element Array Environment

Proc., 1985 Intl. Symp. Antennas Propagat., 20-22 August 1985, Kyoto, Japan, pp. 719-722 1985

A Theory of Mutual Impedances and Multiple Reflections in an N-Element Array Environment

Proc., 1985 Intl. Symp. Antennas and EM Theory, 26-28 August 1985, Beijing, China, pp. 440-443 1985

Interelement Interactions in Phased Arrays: Theory, Methods of Data Analysis, and Theoretical Simulations

NBS Tech. Note 1091

December 1985

Gradient Displacement Errors in Antenna Near-Field Measurements and Their Effects on the Far Field

NBS Tech. Note 1306

October 1986 
MUTH, I. A. (cont.)

Experimental study of Interpanel Interactions at $3.3 \mathrm{GHz}$ Proc., Ant. Meas. Tech. Assoc., 28 September-2 October 1987, seattle, WA, pp. 25-29 1987

Displacement Errors in Antenna Near-Field Measurements and Their Effect on the Far Field

IEEE Trans. Antennas Propagat., Vol. 36, No. 5, pp. 581-591

May 1988

Analytic Correction for Probe-Position Errors in Spherical Near-Field Measurements

Proc. 7th Intl. Conf.' on Antennas and Propagat., 15-18 April 1991, York, England, pp. 762-765 1991

General Analytic Correction for Probe-Position Errors in Spherical NearField Measurements

NIST JRES, Vol. 96 , No. 4, pp. 391-410 July-August 1991

Probe-Position Error Correction in Planar Near-Field Measurement at 60

GHz: Experimental Verification

NIST JRES, Vol. 97 , No. 2, pp. 273-297 March-April 1992

MUTH, I. A.; IEWIS, R. I.

An Iterative Technique to Correct Probe Position Errors in Planar Near-Field to Far-Field Transformations NIST Tech. Note 1323

October 1988

Planar Near-Field Codes for Personal Computers NISTIR 89-3929 October 1989

An Iterative Technique to Correct Probe Position Errors in Planar Near-Field to Far-Field Transformations. Proc., 1989 Intl. Symp. on Antennas and Propagat., 22-25 August 1989, Nagoya, Japan, pp. 901-904. August 1989

A General Technique to Correct Probe Position Errors in Planar NearField Measurements to Arbitrary Accuracy IEEE Trans. Antennas Propagat., Vol. 38, No. 12, pp. 1925-1932 December 1990

Personal Computer Codes for Analysis of Planar Near Fields NISTIR 3970 June 1991

MUTH, L. A.; NEWELI, A. C.; LEWIS, R. L.; CANALES, S.; RREMER, D. P. Experimental \& Theoretical Probe Position Error Correction in Near-Field Antenna Measurements Proc., 12th Annual Meeting \& symp., Ant. Meas. Tech. Assoc., 8-11 oct. 1990, Philadelphia, PA, pp. 13-27 through 13-30 October 1990 
NEWELL, A. C.

Improved Polarization Measurements Using a Modified Three Antenna Technique Proc., 1975 IEEE Intl. Antennas and Propagat. Symp., 2-4 June 1975, Urbana-Champaign, IL, Session 15, pp. 337-340 1975

Improved Polarization Measurements Using a Modified Three Antenna Technique

IEEE Trans. Antennas propagat., spec. Issue on Near-Field scanning Techn., Vol. 36, No. 6, pp. 852-854 June 1988

Error Analysis Techniques for Planar Near-Field Measurements IEEE Trans. Antennas Propagat., Spec. Issue on Near-Field scanning Techn., Vol. 36, No. 6, pp. 754-768 June 1988

Development of Near-Field Test Procedures for Communication satellite Antennas, Phase $I$, part 2 NBSIR $87-3081$ August 1988

NEWELI, A. C.: CRAWFORD, M. L.

Planar Near-Field Measurements on Phased Array Antennas Proc., 1974 Intl. IEEE Ant. Propagat. Soc. Symp., 10-12 June 1974, Atlanta, GA, (Paper 6-7 in 1974 International 1974 IEEE/AP-S Symposium Digest, p. 423)

Planar Near-Field Measurements on High Performance Array Antennas NBSIR 74-380 July 1974

NEWELL, A. C.; RERNS, D. M.

Determination of Both Polarization and Power Gain of Antennas by a Generalized 3-Antenna Measurement Method Electronics Lett., Vol. 7, No. 3, pp. 68-70 January 1971

NEWELI, A. C.; REPJAR, A. G.

Results of Spherical Near-Field Measurements on Narrow-Beam Antennas Proc., 1976 Conf. on Prec. Electromagn. Meas., 28 June-1 July 1976, Boulder, CO, pp. 382-385 1976

Development of Near-Field Test procedures for Communication Satellite Antennas, Phase I, Part 1 NBSIR $85-3031$ September 1985

NEWELL, A. C.; STUBENRAUCH, C. F.

The Effect of Random Errors in Planar Near-Field Measurements Proc., 1986 Intl. IEEE Ant. Propagat. Soc. Symp., 8-13 June 1986, Philadelphia, PA, pp. 195-198 1986

Effect of Random Errors in Planar Near-Field Measurement

IEEE Trans. Antennas Propagat., Spec. Issue on Near-Field scanning Techn., Vol. 36, No. 6, pp. 769-773 June 1988 
NEWEIL, A. C.; YAGHJIAN, A. D.

Study of Errors in Planar Near-Field Measurements

Proc., 1975 IEEE Intl. Antennas and Propagat. Symp.,

2-4 June 1975, Urbana-Champaign, IL, Session 20, pp. 470-473 1975

NEWELL, A. C.; BAIRD, R. C.; WACRER, P. F.

Accurate Measurement of Antenna Gain and Polarization at Reduced

Distances by an Extrapolation Technique

IEEE Trans. Antennas Propagat., Vol. AP-21, No. 4,

pp. 418-431

July 1973

NEWELI, A. C.; FRANCIS, M. H.; RREMER, D. P.

The Determination of Near-Field Correction Parameters for Circularly

Polarized Probes

Proc., 1984 Ant. Meas. Tech. Assoc. Conf.,

2-4 October 1984, Atlanta, GA, pp. 3A3-1 through 3A3-29 1984

NEWELL, A. C.; RREMER, D. P .; GUERRIERI, J. R.

Improvements in Polarization Measurements of Circularly Polarized

Antennas.

Proc., Ant. Meas. Tech. Assoc. Symp., 9-13 October 1989, Monterey, $\mathrm{CA}$, pp. 1-30 through 1-36

October 1989

NEWELL, A. C.; STUBENRAUCH, C. F.; BAIRD, R. C.

Calibration of Microwave Antenna Gain Standards

Proc. IEEE, Spec. Issue on Radio Meas. Methods and Standards,

Vol. 74, Vol. 1, pp. 129-132

January 1986

NEWELI, A. C.; FRANCIS, M. H.; RREMER, D. P.; GRIMM, R. R.

Results of Planar Near-field Testing with Ultralow Sidelobe Antennas

Dig., Intl. IEEE Ant. Propagat. Soc. Symp. on Antennas and

Propagat., 17-21 June 1985, University of British Columbia,

Vancouver, Canada,

pp. 693-698

1985

NEWELI, A. C.; GUERRIERI, J.R.; PERSINGER, R. R.; STILES, J. A.; MCFARLANE, E. J.

Comparison of Antenna Boresight Measurements Between Near-Field and Far-Field Ranges.

Proc., Ant. Meas. Tech. Assoc. Symp., 9-13 October 1989, Monterey, CA, pp. 1-24 through 1-29 October 1989

REEVE, G. R.

Synthesized Isotropic Pattern Antennas for EM Field Measurements

Record, "EMC'81," 1981 IEEE Intl. Symp. on Electromagn. Compat.,

18-20 August 1981, Boulder, CO 1981

REEVE, G. R.; WAINWRIGHT, A. E.

A Frequency Tracking, Tuned, Receiving Monopole

Record, IEEE Ant. Propagat. Soc. Symp., June, 1981 1981 
REPJAR, A. G.; KREMER, D. P.

Results of Planar Near-Field Measurements on a Compact Range at 18 and $54 \mathrm{GHz}$

$$
\text { Dig., IEEE Ant. Propagat. Soc. Symp. }
$$

Accurate Evaluation of a Millimeter Wave Compact Range Using Planar Near-Field Scanning

IEEE Trans. Antennas Propagat., Vol. AP-30, No. 3, pp. $419-425$

May 1982

REPJAR, A. G.; NEWELL, A. C.; BAIRD, R. C.

Antenna Gain Measurements by an Extended Version of the NBS

Extrapolation Method

Dig., 1982 Conf. on Prec. Electromagn. Meas.,

28 June-1 July 1982, Boulder, CO, pp. F-7 through F-9 1982

IEEE Trans. Instrum. Meas., Vol. IM-32, No. 1, pp. 88-91 March 1983

REPJAR, A. G.; NEWELI, A. C.; FRANCIS, M. H.

Accurate Determination of Planar Near-Field Correction Parameters for Linearly Polarized Probes

IEEE Trans. Ant. Propagat., Special Issue on Near-Field Meas.,

Vol. 36 , No. 6 , pp. 855-868

June 1988

REPJAR, A. G.; NEWELL, A. C.; TAMURA, D. T.

Extrapolation Range Measurements for Determining Antenna Gain and Polarization

NBS Tech. Note 1311

August 1987

REPJAR, A. G.; GUERRIERI, J.R.; RREMER, D. P.; CANALES, S. Determining Faults on a Flat Phased Array Antenna Using Planar NearField Techniques

Proc., 13th Annual Meeting \& Symp., Ant. Meas. Tech. Assoc., 7-11 oct. 1991, Boulder, CO, pp. 8-11 through 8-19 October 1991

STUBENRAUCH, C. F.

Some Recent Near-Field Antenna Measurements

Proc., 1979 Antenna Application Symp., September, 1979 1979

STUBENRAUCH, C. F.; FRANCIS, M. H.

Comparison of Measured and Calculated Mutual Coupling in the Near Field Between Microwave Antennas

NBSIR 84-3010

June 1984

Comparison of Measured and Calculated Mutual Coupling in the Near Field Between Microwave Antennas

Proc., 1984 Antenna Applications Symp., 19-21 September 1984,

University of Illinois, Urbana-Champaign, IL, pp. 1-21 1984 
STUBENRAUCH, C. F.; YAGHJIAN, A. D.

Determination of Mutual Coupling Between Co-Sited Microwave Antennas and Calculation of Near-Zone Electric Field

NBSIR $81-1630$

June 1981

STUBENRAUCH, C. F.; SPIESS, W.; GAIIIANO, P. G.; BABJI, T.

International Intercomparison of Electric Field Strength at $100 \mathrm{MHz}$ Dig., 1982 Conf. Prec. Electromagn. Meas.,

28 June-1 July 1982, Boulder, CO, pp. P-3 through P-4 1982

TAGGART, H. E.; SHAFER, J. F.

Testing of Electronic Industries Association Land-Mobile Communication

Antenna Gain Standards at the National Bureau of Standards

IEEE Trans. Veh. Technol. VT-27, pp. 259-264 November 1978

TREADO, M. J.; TAGGART, H. E.; NELSON, R. E.; WORKMAN, J. L.

Fixed and Base Station Antennas

National Institute of Justice (NIJ) Standard-0204.01 (Supersedes

National Institute for Law Enforcement and Criminal Justice

(NILECJ) STD-0204.00, dated November 1977) December 1981

WACKER, P. F.

Near-Field Antenna Measurements Using a Spherical Scan: Efficient Data Reduction with Probe Correction

Dig., Conf. Prec. Electromagn. Meas., No. 113, pp. 286-288

(Institution of Electrical Engineers, London, England) 1974

Antenna Measurements at the National Bureau of Standards Near-Field and Extrapolation Techniques

Proc., 1974 Colloquium on Microwave Communication (5th),

24-30 June 1974, Budapest, PME-113-114. 1974

Non-Planar Near-Field Measurements: Spherical Scanning NBSIR 75-809 1975

Symmetry Analysis Applied to Scattering, Inverse Scattering, and Antenna Patterns: Measurements, Moment Method, and Characteristic Modes

Proc., 1977 Intl. IEEE Ant. Propagat. Soc. Symp., pp. 177-180 1977

Symmetry Analysis Applied to Wave Theory

Dig., Intl. IEEE Ant. Propagat. Soc. Symp. 1977

Recent Results with Spherical Near-Field Antenna Scanning at the National Bureau of Standards

Proc., 1977 European Space Agency Symp., 6-8 June 1977, Noordwijk (Netherlands), pp. 159-164 1977

A Qualitative Survey of Near-Field Analysis and Measurement NBSIR 79-1602

January 1979 
WACRER, P. F.(cont.)

Plane-Radial Scanning Techniques With Probe Correction; Natural

Orthogonalities With Respect to Summation on Planar Measurement Lattices

Dig., IEEE Ant. Propagat. Soc. Symp.

February 1979

WACKER, P. F.; NEWELI, A. C.

Advantages and Disadvantages of Planar, Circular Cylindrical and

Spherical Scanning and Description of the NBS Antenna Scanning

Facilities

Proc., 1977 European Space Agency Symp., 6-8 June 1977, Noordwijk,

Netherlands, pp. 115-121 1977

WAIT, D. F.

Satellite Earth Terminal G/T Measurements

Microwave Journal, Vol. 20, No. 4, pp. 49, 51, 58 April 1977

Earth Terminal Measurement system Operations Manual

NBSIR 78-879

April 1978

Precision Measurement of Antenna System Noise Using Radio Stars

Dig., 1982 Conf. Prec. Electromagn. Meas., 28 June-1 July 1982,

Boulder, CO, p. F-17 1982

IEEE Trans. Instrum. Meas., Vol. IM-32, No. 1, pp. 110-116 March 1983

Earth Terminal Measurement System Operations Manual (Revised)

NBSIR 83-1679(R) January 1983

WAIT, J. R.; HILL, D. A.

Fields of a Horizontal Loop of Arbitrary shape Buried in a Two-Layer Earth

Radio Science, Vol. 15, No. 5, pp. 903-912 september-October 1980

WILSON, P. F.; CBANG, D. C.; MA, M. T.

Input Impedance of a Probe Antenna Exciting a TEM Cell

NBS Tech. Note 1054

April 1982

WITIMANN, R. C.

Probe Correction in Spherical Near-field scanning Viewed as an Ideal

Probe Measuring an Effective Field

Dig., 1984 Intl. Symp. Ant. Propagat., 25-29 June 1984, Boston, MA, pp. 674-677

1984

Spherical Wave Operators and the Translation Formulas

IEEE Trans. Antennas Propagat., Vol. 36, No. 8, pp. 1078-1087 August 1988

Comments on Spherical Near-Field Antenna Measurements:

Review for Ant. Propagat. Soc. Newsletter, Vol. 31 , No. 3, pp. $35-36$

June 1989 
WITTMANN, R. C. (cont.)

Spherical Near-Field Scanning: Determining the Incident Field Near a Rotatable Probe.

Proc. IEEE Intl. Symp. Ant. Propagat. Soc., 7-11 May 1990, Dallas, TX, pp. 224-227 May 1990

Probe-Corrected Spherical Near-Field Scanning Theory in Acoustics Conf. Record, IEEE Instrumentation/Measurement Technology Conf., 14-16 May 1991, Atlanta, GA, pp. 283-286 May 1991

IEEE Trans. Iintrum. Metrol., Vol. IM-41, No. 1, pp.17-21 February 1992

WITTMANN, R. C.; STUBENRAUCH, C. F.

Spherical Near-Field Scanning: Experimental and Theoretical studies NISTIR 3955 July 1990

WITTMANN, R. C.; YAGHJIAN, A. D.

Spherical-Wave Expansions of Piston-Radiator Fields

J. Acoust. Soc. Amer., Vol. 30, No. 3, pp. 1647-1655 September 1991

WYSS, J. C.; SHEERAN, S.

A Passive, Optical Modulator and Link for Antennas

IEEE J. Lightwave Technol., Vol. LT-3, No. 2, pp. 316-321 April 1985

WYSS, J. C.: ANSON, W. J.; ORR, R. D.

Building Penetration Project NBSIR 84-3009

September 1984

WYSS, J. C.; KANDA, M.; MELQUIST, D. G.; ONDREJKA, A. R. )

Optical Modulator and Link for Broadband Antennas

Dig., 1982 Conf. Prec. Electromagn. Meas.,

28 June-1 July 1982, Boulder, CO, pp. P-16 through P-17 1982

YAGHJIAN, A. D.

Upper-Bound Errors in Far-Field Antenna Parameters Determined from

Planar Near-Field Measurements. Part 1: Analysis

NBS Tech. Note 667

October 1975

A Direct Approach to the Derivation of Electric Dyadic Green's Functions

Dig., Ant. Propagat. Soc. Symp., October, 1976, pp. 76-156

October 1976

NBS Tech. Note 1000

December 1977

Near-Field Antenna Measurements on a Cylindrical Surface: A Source Scattering-Matrix Formulation

NBS Tech. Note 696

September 1977

An Approximate Expression for the Principal Beamwidth of Directive Antennas in Terms of Aperture Fields

NBSIR 81-1644

March 1981 
YAGHJIAN, A. D. (cont.)

A Delta-Distribution Derivation of the Electric Field in the source Region

Dig., 1981 IEEE Ant. Propagat. Soc. Symp. June 1981

J. Electromagn. Soc., Vol. 2, No. 2, pp. 161-167 April-June 1982

Efficient Computation of Antenna Coupling and Fields Within the Near-Field Region

IEEE Trans. Antennas Propagat., Vol. AP-30, No. 1, pp. 113-128

January 1982

Approximate Formulas for the Far Field and Gain of Open-Ended Rectangular Waveguide

NBSIR 83-1689

May 1983

YAGHJIAN, A. D.; WITMMANN, R. C.

The Receiving Antenna as a Linear Differential Operator: Application to Spherical Near-Field Scanning

IEEE Trans. Antenna Propagat., Vol. AP-33, No. 11, pp. 1175-1185

November 1985 


\section{DIELECTRIC MEASUREMENTS}

BARER-JARVIS, J R.

Transmission/Reflection and short-Circuit Line Permittivity Measurement Methods

NIST Tech. Note 1341
July 1990

BARER-JARVIS, J.R.; GEYER, R. G.

Improvements in Transmission Line Permittivity and Permeability Measurements

Dig., Conf. Prec. Electromagn. Meas., 11-14 June 1990, Ottawa, Canada, pp. 232-233 June 1990

BARER-JARVIS, J.R.; VANZURA, E. J.; RISSICK,. . A. Improved Technique for Determining Complex Permittivity with the Transmission/Reflection Method

IEEE Trans. Microwave Theory Tech., Vol. 38, No. 8, pp. 1096-1103 August 1990

BENNETT, W. S.; TAGGART, H. E.

Characteristics of A CISPR/VDE Far-Field EMI Test site with Ground screen

Proc., Third Rotterdam Electromagn. Compat. Symp., December 1978

1978

BUSSEY, H. E.

Measurement of RF Properties of Materials, A Survey

Proc. IEEE, Special Issue on Radio Meas. Methods and Standards,

Vol. 55, No. 6, pp. 1046-1053

June 1967

Rapport sur la Comparaison Int'l des Mesures de Permittivite Complexe a

$9 \mathrm{GHz}$ (Intl. Comparison of Complex Permittivity Measurement at $9 \mathrm{GHz}$ )

Proc., of Comite Consultatif, D'Electricite Comite, Bureau Int.l des Poids et Mesures, 12-13 October 1972, Sevres, France, pp. $124-137$

1972

Wavelength of a slotted Rectangular Line Containing Two Dielectrics NBSIR 73-326

July 1973

Dielectric Measurements of Lunar Soil

Proc., 1979 Lunar and Planetary Science Conf. (10th)

Natl. Aeronautics and space Administration, 19-23 March 1979,

Lyndon B. Johnson space Center, Houston, TX 1979

Open Circuited Coaxial Resonator for High Sensitivity Dielectric Measurements, Application to Lunar Soil 70051-20

Proc., 1979 Lunar and Planetary Science Conf. (10th)

Natl.Aeronautics and space Administration, 19-23 March 1979,

Lyndon B. Johnson space center, Houston, TX 1979

Dielectric Measurements in a Shielded Open Circuit Coaxial Line

IEEE Trans. Instrum. Meas., Vol. IM-29, No. 2, pp. 120-124 June 1980 
BUSSEY, H. E.; RICHMOND, J. H.

Scattering by a Lossy Dielectric Circular Cylindrical Multilayer, Numerical Values

IEEE Trans. Antennas Propagat., Vol. 23, No. 5, pp. 723-725 September 1975

BUSSEY, H. E.; MORRIS, D.; ZALTSMAN, E. B.

International Comparison of Complex Permittivity Measurement at $9 \mathrm{GHz}$

IEEE Trans. Instrum. Meas., Vol. IM-23, No. 3, pp. 235-239 September 1974

DAYWITT, W. C.

Complex Permittivity of Beryllium Oxide Between 100 and 300 Kelvins at 9.3 Gigahertz

IEEE Trans. Instrum. Meas., Vol. IM-34, No. 1, pp. 98-99 March 1985

DOMICH, P. D.; BARER-JARVIS, J.R.; GEYER, R. G.

Optimization Techniques for Permittivity and Permeability Determination NIST JRES, Vol. 96 , No. 5, pp. 565-575

September/October 1991

ELLERBRUCH, D. A.; LITTLE, W. E.; BOYNE, B. S.; BACHMAN, D. D. Microwave Characteristics of snow

Proc., 1977 Western Snow Conf., 18-21 April 1977,

Albuquerque, NM, p 7 1977

GEYER, R. G.

Dielectric. Mixing Rules for Background Test Soils

NBSIR 88-3095

June 1988

Electrodynamics of Materials for Dielectric Measurement Standardization. Record, Instrum. Meas. Tech. (IMTC) Conf., 13-15 February 1990, San Jose, CA, pp. 2-7 February 1990

Dielectric Characterization and Reference Materials

NIST Tech. Note 1338

April 1990

GEYER, R. G.; WEIL, C. M.; RISSICR, W. A.

Precision Dielectric Measurements Using a Mode-Filtered Cylindrical Cavity Resonator

Dig., Conf. Prec. Electromagn. Meas., 11-14 June 1990, Ottawa,

Canada, pp. 174-175 June 1990

GEYER, R. G.; JANEZIC, M. D.; RISSICK, W. A.; BARER-JARVIS, J.R.

Preliminary Microwave Complex Permittivity Measurements of Heavy-Metal Fluoride Glasses

Proc., Third DoD Symp. on Electromagnetic Windows, 14-16 Nov.

1989, Redstone Arsenal, Huntsville, AL, pp. 116-141 1989 
HILL, D. A.

Near-Field Detection of Buried Dielectric objects

IEEE Trans. Geosci. Remote Sensing, Vol. 27, No. 4, pp. 364-368 July 1989

Electric and Magnetic Dipole Radiation in a Random Medium

Electromagnetics, Vol. 10, pp. 279-292 1990

HILL, D. A.; RANDA, M.; LARSEN, E. B.; KOEPRE, G. B.; ORR, R. D. Generating standard Reference Electromagnetic Fields in the NIST Anechoic Chamber, 0.2 to $40 \mathrm{GHz}$

NIST Tech. Note 1335 March 1990

JANEZIC, M. D.; GROSVENOR, J. H.

Improved Technique for Measuring Permittivity of Thin Dielectrics with a Cylindrical Resonant Cavity

Conf. Record, IEEE Instrument. Meas. Tech. Conf., 14-16 May 1991, Atlanta, GA, pp. 580-584 May 1991

JESCB, R. L.

Dielectric Measurements of Five Different Soil Textural Types as Functions of Frequency and Moisture Content NBSIR 78-896 1978

Dielectric Measurements of Oil Shale as Functions of Temperature and Frequency

IEEE. Trans. Geosci. Remote Sensing, Vol. GE-22, No. 2, pp. 99-105 March 1984

Measurement of Shielding Effectiveness of Different Cable and Shielding Configurations by Mode-Stirred Techniques

IEEE Trans. Electromagn. Compat., Vol. EMC-30, pp. 222-228 August 1988

JONES, R. N.; BUSSEY, H. E.; LITTLE, W. E.; METZRER, R. F.

Electrical Characteristics of Corn, Wheat, and soya in the $1-200 \mathrm{MHz}$ Range

$$
\begin{array}{ll}
\text { NBSIR } & 78-897 \\
& \text { October } 1978
\end{array}
$$

LAWTON, R. A.

Autocorrelation and Power Measurement with Pyroelectric and Dielectric Bolometers

IEEE Trans. Instrum. Meas., IM-22, pp. 299-306 December 1973

LAWTON, R. A.; ANDERSON, W. T. Two Layer Dielectric Microstrip Line Structure: $\mathrm{SiO}_{2}$ on $\mathrm{Si}$ and GaAs on Si: Modeling and Measurement

IEEE Trans. Microwave Theory Tech., Vol. 36,

No. 4, pp. 785-789 April 1988

WEIL, C. M.; KISSICK, W. A.

The NIST Electromagnetic Properties of Materials Program

Conf. Record, IEEE Instrumentation \& Measurement Technology Conf., 14-16 May 1991, Atlanta, GA, pp. 626-630

May 1991 
VANZURA, E. J.; RISSICR, W. A.

Advances in NIST Dielectric Measurement Capability Using a Mode-Filtered Cylindrical Cavity

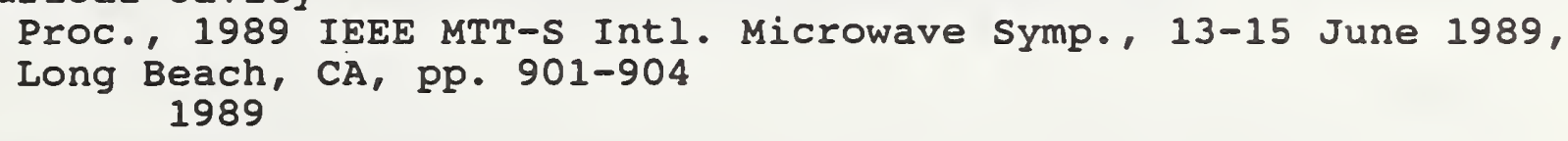

VANZURA, E. J.; ROGERS, J. E.

Evaluation of a Resonant Circuit Model Using Reflected S-Parameter Data Conf. Record, IEEE Instrumentation \& Measurement Technology Conf., 14-16 May 1991, Atlanta, GA, pp. 150-155

May 1991 
ADAMS, J . W.

Measurement of Electromagnetic Radiation from Electric Rail Cars NBSIR 82-1669

August 1982

ADAMS, J. W. FRIDAY, D. S.

Measurement Procedures for Electromagnetic Compatibility Assessment of Electroexplosive Devices

IEEE Trans. Electromagn. Compat., Vol. 30, No.4,

pp. 484-494

November 1988

ADAMS, J. W.; TAGGART, H. E.

Electromagnetic Compatibility Evaluation of Seven Electronic Brake systems

NBSIR 78-872

February 1978

ADAMS, J. W.: VANZURA, E. J.

Shielding Effectiveness Measurements of Plastics

NBSIR 85-3035

January 1986

EMC Technology and Interference Control News, pp. 39-44 September-October 1986

ADAMS, J. W.; BENSEMA, W. D.; RANDA, M.

Electromagnetic Noise in Grace Mine

NBSIR 74-388

June 1974

ADAMS, J. W.; BENSEMA, W. D.; TOMOEDA, N. C.

Surface Magnetic Field Noise Measurements at Geneva Mine

NBSIR 74-369

June 1974

ADAMS, J. W.; CRAWFORD, M. L.; SHAFER, J. F.

Electromagnetic Interference (EMI) Measurements for Automotive Applications

Proc., 1976 SAE Automotive Congress Conf., 23-27 February, 1976, Detroit, $M I$, pp. $1-6$ 1976

ADAMS, J.W.; TAGGART, H. E.; SPAULDING, A. D.

Survey Report of the U.S. Bureau of Mines Electromagnetic Noise Measurement Program

NBS Rept. 10723

November 1971

ADAMS, J. W. ; WU, D. I.; BUDLONG, A.

Measurement of Electric Field Strength Near Higher-Powered Personal

Transceivers

NISTIR 90-3938

May 1990

ADAMS, J. W.; KANDA, M.; SHAFER, J. F.; WU, Y.

Near-Field Electric Field Strength Levels of EM Environments Applicable to Automotive systems

Proc., 1977 IEEE Intl. Symp. on Electromagn. Compat.,

2-4 August 1977, Seattle, WA, pp. 336-343 1977 
ALSPACH, W. J.; MILIER, C. R. S.; REEVE, G. R.

An Assessment of Electromagnetic Interference (EMI) and Electromagnetic Compatibility (EMC) Measurement Practices to Meet U.S. Army Aviation Research and Development Command EMI/EMC Requirements NBSIR 84-1698(R) January 1984

ANDREWS, J. R.

Picosecond Pulse Generators Using Microminiature Mercury Switches NBSIR 74-377 1974

Impulse Generator Spectrum Amplitude Measurement Techniques IEEE Trans. Instrum. Meas., Vol. IM-25, No. 4, pp. 380-384 December 1976

ANDREWS, J. R.; ARTHUR, M. G.

Spectrum Amplitude--Definition, Generation and Measurement NBS Tech. Note 699 October 1977

ANDREWS, J. R.; BAIDWIN, E. E. UHF Impulse Generator NBSIR 78-880

April 1978

SHF Impulse Generator NBSIR 78-888 June 1978

ARTHUR, M. G.

Impulse spectral Intensity -- What is it? NBSIR $\begin{aligned} 74-365 \\ \text { May } 1974\end{aligned}$

Proc., 1978 Electromagn. Interference Workshop NBS SP 551 July 1979

ARTHUR, M. G.; ORR, R. D.; REEVE, G. R.

Planning Guidance for Future EMI Measurement Instrumentation NBSIR 82-1662

April 1982

BAIRD, R. C.

Methods of Calibrating Microwave Hazard Meters

Proc., Intl. Symp on Biological Effects and Health Hazards of Microwave Radiation, October, 1973, Warsaw, Poland, pp. 228-236 1973

BELSHER, D. $R$.

Development of Near-Field Electric Energy Density Meter Model EDM-2 Department of Health, Education, and Welfare, Publ. No. (NIOSH) 75-140

March 1975

BENSEMA, W. D.

Coal Mine Magnetic-Field Noise Measurements Proc., 1972 W. Virginia Conf. on Coal Mine Electrotechnology (1st), 2-4 August 1972, West Virginia Univ., Morgantown, pp. XII-1 through XII-12

1972 
BENSEMA, W. D. (cont.)

A Noise Spectrum Measurement System Using the Fast Fourier Transform IEEE Trans. Electromagn. Compat., Vol. EMC-19, No. 2, pp. 37-43

$$
\text { May } 1977
$$

Amplitude, Time, and Frequency Statistics of Quasi-Impulsive Noise Proc., 1977 Electromagn. Compat. Symp., 18-30 June 1977, Montreux, Switzerland, pp. 347-352 1977

Broadband Orthogonal Array Antenna System: Microprocessor Control and Computation

Record, "EMC '81," 1981 IEEE Intl. Symp. on Electromagn. Compat., 18-20 August 1981, Boulder, CO 1981

Handbook for Broadband Isotropic Antenna system

Volume 1 - Operations Manual

NBSIR 83-1693

July 1983

BENSEMA, W. D.: KANDA, M.; ADAMS, J.W.

Electromagnetic Noise in Robena No. 4 Coal Mine NBS Tech. Note 654

April 1974

Electromagnetic Noise in Itmann Mine

NBSIR 74-390

June 1974

BENSEMA, W. D.; KOEPRE, G. H.; MEDLEY, H. W.

Handbook for NBS Multisensor Automated EM Field Measurement system NBSIR 86-3056

October 1986

BENSEMA, W. D.; REEVE, G. R.; KOEPRE, G. H.

A Multisensor Automated EM Field Measurement system

Proc., 1985 Instrum. and Meas. Technol. Conf., 20-22 March 1985, Tampa, FL, pp. 200-202 1985

BOWMAN, R. R.

Field Strength Above $1 \mathrm{GHz}$ : Measurement Procedures for Standard Antennas Proc., IEEE, special Issue on Radio Meas. Methods and Standards, Vol. 55, No. 6, pp. 981-990 June 1967

Quantifying Hazardous Electromagnetic Microwave Fields: Practical Considerations

Proc., 1969 Biological Effects and Health Implications of Microwave Radiation, 17-19 September 1969, Richmond, VA., BRH/DBE 70-2, U.S. Dept. of Health, Education, and Welfare, Rockville, MD., pp. 204-209

June 1970 
BOWMAN, R. R. (cont.)

Some Recent Developments in the Characterization and Measurement of Hazardous Electromagnetic Fields

Proc., 1973 Intl. Symp. Biologic Effects and Health Hazards of Microwave Radiation, 15-18 october 1973, Warsaw (Poland), pp. 217-227

1973

A Probe for Measuring Temperature in Radio-Frequency-Heated Material IEEE Trans. Microwave Theory Technol., Vol. MTT-24, No. 1 , pp. 43-45 January 1976

Calibration Techniques for Electromagnetic Hazard Meters: $500 \mathrm{MHz}$ To $20 \mathrm{GHz}$ NBSIR 75-805

April 1976

A Temperature Probe for Radio-Frequency Heated Material NBSIR 81-1634

January 1981

CAMELL, D. G.; IARSEN, E. B.; ANSON, W. J.

NBS Calibration Procedures for Horizontal Dipole Antennas ( 25 to $100 \mathrm{MHz}$ )

Proc. IEEE Intl. Symp. on Electromagn. Compat., 2-4 August 1988, Seattle, WA, pp. 390-394 1988

CAMELL, D. G.; LARSEN, E. B.; CRUZ, J. E.; HILL, D. A.

NIST Calibration Procedure for Vertically Polarized Monopole Antennas, $30 \mathrm{kHz}$ to $300 \mathrm{MHz}$

NIST Tech. Note 1347

January 1991

CAVCEY, R. H.

Transmission Loss Through 6061 T-6 Aluminum Using a Pulsed Eddy Current Source

"Materials Evaluation", American Society for Nondestructive Testing, Vol. 47 , No. 2, pp. 216-218 February 1989

CAVCEY, R. H.; FRIDAY, D. S.

Aircraft Field Degradation and Electromagnetic Compatibility NBSIR 88-3083

January 1988

CLARK, H. E.

Requirements for an Effective National Nonionizing Radiation Measurement system

NBS SP 613

June 1981

CRAWFORD, M. I.

Evaluation of Reflectivity Level of Anechoic Chambers Using Isotropic, 3-Dimensional Probing

Proc., 1974 Intl. IEEE Ant. Propagat. Soc. Symp., 10-12 June 1974, Atlanta, GA, pp. 28-34

1974 
CRAWFORD, M. I. (cont.)

Generation of Standard EM Fields Using TEM Transmission Cells IEEE Trans. Electromagn. Compat., Vol. EMC-16, No. 4, pp. $189-195$

November 1974

Generation of standard EM Fields for Calibration of Power Density Meters $20 \mathrm{kHz}$ to $1000 \mathrm{MHz}$

NBSIR 75-804

January 1975

Techniques for Measurement of Electromagnetic Radiation and Susceptibility of Electronic Equipment

Proc., 1975 symp. and Technical Exhib. on Electromagn. Compat. (1st), 20-22 May 1975, Montreux, Switzerland, pp. $38-44$

1975

Experimental Evaluation of the Radiation Characteristics of Dipole Sources Enclosed in a TEM Transmission Cell

Proc., 1976 Conf. on Precision Electromagn. Meas., 28 June-1 July 1976, Boulder, CO, pp. 57-59 1976

Improved Techniques and Instrumentation for EMC Measurements IEEE 1976 Intl. Symp. on Electromagn. Compat., 13-15 July 1976, Washington, DC 1976

Comparison and Selection of Techniques for Measuring EM Radiated Emissions and Susceptibility of Large Equipment Proc., 1979 Symp. and Technical Exhib. on Electromagn. Compat. (3rd), 1-3 May 1979, Rotterdam, Netherlands, pp. 115-122 1979

Evaluation of a Reverberation Chamber Facility for Performing EM Radiated Fields susceptibility Measurements

NBSIR 81-1638 February 1981

Options to Open-Field and Shielded Enclosure Electromagnetic Compatibility Measurements

Proc., 1981 Intl. Electromagn. Compat. Symp., 10-12 March 1981, Zurich, Switzerland 1981

Improving the Repeatability of EM Susceptibility Measurements of Electronic Components When Using TEM Cells

SAE Technical Paper Series, 830607, International Congress and Exposition, 28 February-4 March 1983, Detroit, MI, pp. $1-8$

1983

Evaluation of Shielded Enclosure for EMI/EMC Measurements Without and With RF Anechoic Material

Proc., 1983 Electromagn. Compat. Symp. and Exhib., 8-10 March 1983, Zurich, Switzerland, pp. 397-402 1983 
CRAWFORD, M. I. (cont.)

Comparison of Open-Field, Anechoic Chamber and TEM Cell

Facilities/Techniques for Performing Electromagnetic Radiated Emissions Measurements

Record, IEEE 1983 Intl. Symp. on Electromagn. Compat., 23-25

August 1983, Arlington, VA, pp. 413-418 1983

A TEM Driven Reverberating Chamber: A Single Facility for Radiated

EMS/V Testing, $10 \mathrm{kHz}-18 \mathrm{GHz}$ ?

Proc., EMC Expo 1987, Intl. Conf. on Electromagn. Compat., 19-21

May 1987, San Diego, CA, pp. T11.18 through T11.28 1987

TEM/Reverberating Chamber Design/Concept study: A Single Facility for

Large system Radiated EMC Testing, $10 \mathrm{kHz}-40 \mathrm{GHz}$ ?

Proc., EMC EXPO, 1-3 August 1989, Washington, DC,

pp. B6.22-B6.29

1989

CRAWFORD, M. I.; BEAN, J. L.

NSWC Reverberating Chamber: A High Power Microwave Exposure Chamber Proc., 3rd Natl.High Power Microwave Technical Conf.,

1-5 December 1986, Kirtland AFB, NM, p. 5 1986

CRAWFORD, M. L.; KOEPRE, G. H.

Performing EM Susceptibility/Vulnerability Measurements Using a

Reverberation Chamber

Proc., EMC EXPO 1986, Intl. Conf. on Electromagn. Compat.,

16-19 June 1986, Washington, DC, pp. T28.7-T28.14 1986

Proc., 7th Intl. Zurich Symp. and Technical Exhib. on Electromagn.

Compat., 3-5 March 1987, Zurich, Switzerland, pp. 121-126 1987

Operational Considerations of a Reverberation Chamber for EMC Immunity Measurements, Some Experimental Results

Proc., 1984 IEEE Natl.Symp. on Electromagn. Compat.,

24-26 April 1984, San Antonio, TX, pp. 47-54 1984

Comparing EM Susceptibility Measurement Results Between Reverberation and Anechoic Chambers

Record, IEEE 1985 Intl. Symp. on Electromagn. Compat.,

20-22 August 1985, Wakefield, MA, pp. 200-202

1985

Design, Evaluation and Use of a Reverberation Chamber for Performing Electromagnetic susceptibility/Vulnerability Measurements NBS Tech. Note 1092

April 1986 
CRAWFORD, M. L.; ROEPKE, G. H. (cont.)

EMR Test Facilities - Evaluation of Reverberation Chambers Located at NSWC, Dahlgren, VA

NBSIR 86-3051

June 1986

Preliminary Evaluation of Reverberation Chamber Method for Pulsed RF Immunity Testing

Record, IEEE Intl. Symp. on Electromagn. Compat.,

16-18 September 1986, San Diego, CA, pp. 270-278 1986

CRAWFORD, M. L.; IADBURY, J. M.

Mode-stirred Chamber for Measuring Shielding Effectivess of Cables \& Connectors An Assessment of MIL-STD 1344A Method 3008

Proc., 1988 IEEE Intl. Symp. on Electromagn. Compat.,

1-5 August 1988, Seattle, WA, pp. 30-36 1988

CRAWFORD, M. L.; RIDDLE, B. F.

A Proposed TEM Driven Mode-stirred Chamber for Large System Radiated EMC/V Testing $10 \mathrm{kHz}-40 \mathrm{GHz}$

Proc., 9th Intl. Symp, and Tech. Exhibition on EMC

12-14 March 1991, Zurich, Switzerland, pp. 431-437 1991

CRAWFORD, M. L.; TBOMAS, C. L.

Converting a Rectangular shielded Enclosure into a TEM Transmission Cell for EMI Measurements

IEEE 1977 Intl. Symp. on Electromagn. Compat., 2-5 August 1977, Seattle, WA

1977

CRAWFORD, M. L.; WORKMAN, J. L.

Asymmetric Versus symmetric TEM Cells for EMI Measurements

Proc., 1978 IEEE Intl. Symp. on Electromagn. Compat., 20-22 June 1978, Atlanta, GA, pp. 204-210 1978

Using a TEM Cell for EMC Measurements of Electronic Equipment NBS Tech. Note 1013 April 1979

Predicting Free-Space Radiated Emissions from Electronic Equipment Using TEM Cell and Open-Field Site Measurements

Record, 1980 Intl. Electromagn. Compat. Symp. 1980

Spherical Dipole for Radiating standard Fields

Dig., 1980 Conf. Prec. Electromagn. Meas.

June 1980

CRAWFORD, M. L.; KOEPRE, G. B.; LADBURY, J. M.

EMR Test Facilities Evaluation of Reverberating Chamber Located at RADC, Griffiss, AFB, Rome, New York NBSIR $87-3080$

December 1987

CRAWFORD, M. L.; WORKMAN, J. L.; THOMAS, C. L.

Generation of EM Susceptibility Test Fields Using a Large

Absorber-Loaded TEM Cell

IEEE Trans. Instrum. Meas., Vol. IM-26, No. 3, pp. 336-343

September 1977 
CRAWFORD, M. L.; WORKMAN, J. L.; THOMAS, C. I.

Expanding the Bandwidth of TEM Cells for EMC Measurements IEEE Trans. Electromagn. Compat., Vol. EMC-20, No. 3, pp. 368-375

August 1978

CRAWFORD, M. L.; LADBURY, J. M.; RIDDLE, B. F.; LARSEN, E. B.

Electromagnetic Radiation Test Facilities: Evaluation of a Small

Reverberating Chamber Located at RADC, Griffiss AFB, Rome, NY NISTIR 90-3939

June 1990

CRAWFORD, M. I.; MA, M. T.; LADBURY, J. M.; RIDDLE, B. F .

Measurement \& Evaluation of a TEM/Reverberating Chamber NIST Tech. Note 1342

July 1990

CRUZ, J. E.

Design, Construction, and Calibration of the Broadband Electric Field Monitor (EFM-5)

Record, "EMC '81," 1981 IEEE Intl. Symp. on Electromaggn. Compat., 18-20 August 1981, Boulder, CO 1981

CRUZ, J. E.; IARSEN, E. B.

Screenroom Measurements of Antenna Factors

Proc., 1985 Instrum. and Meas. Technol. Conf., 20-22 March 1985,

Tampa, FL, p. 208 1985

Assessment of Errors for MIL-STD-461/462

NBS Tech. Note 1300

October 1986

Alternative Techniques for some Typical MIL-STD-461/462 Types of

Measurements

NBS Tech. Note 1320

March 1989

CRUZ, J. E.; DRIVER, L. D.; RANDA, M.

Design of the National Bureau of Standards Isotropic Magnetic Field

Meter (MFM-10) $300 \mathrm{kHz}$ to $100 \mathrm{MHz}$

NBS Tech. Note 1085

October 1985

DECRER, W. F.; CRAWFORD, M. I.; WILSON, W. A.

Construction of a Large Transverse Electromagnetic Cell NBS Tech. Note 1011

February 1979

DRIVER, I. D.; CRUZ, J.E.

Development of the NBS Isotropic Magnetic-Field Meter (MFM-10), $300 \mathrm{kHz}$ to $100 \mathrm{MHz}$

Proc., 1982 Intl. Symp. on Electromagn. Compat., 8-10 September

1982, Santa Clara, CA, pp. 460-467 1982 
DRIVER, L. D. ; RANDA, M. An Optically Linked Electric and Magnetic Field Sensor for Poynting Vector Measurements in the Near Fields of Radiating Sources IEEE Trans. Electromagn. Compat., Vol. 30, No. 4, pp. 495-503 November 1988

DRIVER, L. D.; REEVE, G. R. Application of a Systematic Approach to an Investigation of $\mathrm{HF}$ Interference to a Shipboard Radar set Proc., 1984 IEEE Natl.Symp. on Electromagn. Compat., 24-26 April 1984, San Antonio, TX, pp. 211-218 1984

FITZGERRELL, R. G.

Experimental RF Field Polarization Meter FAA-RD-74-188 1975

Free-Space Transmission Loss for Anechoic Chamber Performance Evaluation Record, "EMC '81," 1981 IEEE Intl. Symp. on Electromagn. Compat., 18-20 August 1981, Boulder, CO 1981

IEEE Trans. Electromagn. Compat., Vol. EMC-24, No. 3, pp. 356-358 August 1982

A Partial Loop Source of E \& H Fields for Antenna Factor Calibration (A Loop Cell)

Proc., 1982 Antenna Meas. Techniques Assoc., 5-7 October 1982, New Mexico State University, Las Cruces, NM, pp. 15-1 through 15-22 1982

E-Fields Over Ground

Record, IEEE 1983 Intl. Symp. on Electromagn. Compat., 23-25

August 1983, Arlington, VA, pp. 6-9 1983

A Source of E\&H Fields for Antenna-Factor Calibration

IEEE Trans. Electromagn. Compat., Vol. EMC-26,

No. 2, pp. 58-65

May 1984

Site Attenuation

Record, IEEE 1985 Intl. Symp. on Electromagn. Compat.,

20-22 August 1985, Wakefield, MA, pp. 612-617 1985

NBS Tech. Note 1089

November 1985

IEEE Trans. on Electromagn. Compat., Vol. EMC-28,

No. 1 , pp. 38-40

February 1986

Monopole Impedance and Gain Measurements on Finite Ground Planes

IEEE Trans. on Antennas and Propat., Vol. 36,

No. 3, pp. 431-438

March 1988 
FITZGERRELI, R. G. (cont.)

Monopole Impedance and Gain Measurements of Finite Ground Planes National Institute of Justice Report, 200-87 May 1989

FLANIGAN, W. F. Jr.; BOWMAN, R. R.; LOWELL, W. R. Nonmetallic Electrode system for Recording EEG and ECG in Electromagnetic Fields Physiology and Behavior, Vol. 18, No. 3, pp. 531-533 1977

FRIDAY, D. S. Methodology for Statistical Control of the Anechoic Chamber Field Generation System NBSIR 85-3033 January 1986

FRIDAY, D. S.; ADAMS, J. W.

A Statistical Characterization of Electroexplosive Devices (EED) Relevant to Electromagnetic Compatibility (EMC) NBS Tech. Note 1094 May 1986

GREENE, F . M.

NBS Field-Strength Standards and Measurements ( $30 \mathrm{MHz}$ to $1000 \mathrm{MHz}$ ) Proc., IEEE Spec. Issue on Radio Measurement Methods and Standards, Vol. 55, No. 6, pp. 970-981 June 1967

Development and Construction of an Electromagnetic Near-Field Synthesizer NBS Tech. Note 652

May 1974

Development of Electric and Magnetic Near-Field Probes NBS Tech. Note 658 January 1975

Measurement of RF Power-Absorption in Biological specimens (10 to $100 \mathrm{MHz}$ )

$$
\begin{array}{r}
\text { NBS Tech. Note } 687 \\
\text { November } 1976
\end{array}
$$

BILI, D. A.

Theory of Near-field Phased Arrays for Electromagnetic Susceptibility Testing

NBS Tech. Note 1072

February 1984

Electromagnetic Wave Propagation in an Asymmetrical Coal seam

IEEE Trans. Antennas Propagat., Vol. AP-34, No. 2,

pp. 244-247

February 1986

A Numerical Method for Near-Field Array Synthesis

IEEE Trans. Electromagn. Compat., Vol. EMC-27, No. 4, pp. 201-211 November 1985

Radio-Wave Propagation from a Forest to a Clearing Electromagnetics, pp. 217-228 1986 
HILI, D. A. (cont.)

Out-of-Band Response of a Coax-to-Waveguide Adapter

IEEE Trans. Electromagn. Compat., Vol. EMC-28, No. 3, pp. 156-158

August 1986

An Error Bound for Near-Field Array Synthesis

IEEE Trans. Electromagn. Compat., Vol. EMC-28, No. 4, pp. 273-276

November 1986

Effect of a Thin Conducting Sheet on the Fields of a Buried Magnetic Dipole

Electromagnetics, pp. 71-79 1987

A Circular Array for Plane-Wave Synthesis

IEEE Trans. Electromagn. Compat., Vol. EMC-30, No. 1, pp. 3-8 February 1988

Electromagnetic Scattering by Buried Objects of Low Contrast

IEEE Trans. Geosci. Remote Sensing, Vol. 26,

No. 2, pp. 195-203

March 1988

Electromagnetic Detection of Long Conductors in Tunnels

Proc., Third Tunnel Detection Symp., 12-15 January 1988,

Golden, CO, pp.518-537 1988

Reflection Coefficient of a Waveguide with Slightly Uneven Walls

IEEE Trans., Microwave Theory Tech., Vol MTT-37,

No. 1, pp. 244-252 January 1989

Clutter Models for Subsurface Electromagnetic Applications NISTIR 89-3909

February 1989

Quasi-Static Analysis of a Two-Wire Transmission Line Located at an Interface

Radio Science, Vol. 25, No. 4, pp. 435-440 July-August 1990

Near-Field and Far-Field Excitation of a Long Conductor in a Lossy Medium

$$
\begin{aligned}
& \text { NISTIR } 3954 \\
& \text { September } 1990
\end{aligned}
$$

A Generalization of the Cornu Spiral for Lossy Media

J. Appl. Phys., Vol. 69, No. 3, pp. 1772-1774

February' 1991

Diffraction by a Half Plane in a Lossy Medium

J. Appl. Phys., Vol. 69, No. 12, pp. 8405-8407

June 1991

Gradiometer Antennas for Tunnel Detection

NISTIR 3990

June 1992 
BILL, D. A.; ROEPRE, G. B.

A Near-field Array of Yagi-Uda Antennas for Electromagnetic susceptibility Testing

NBS Tech. Note 1082

July 1985

IEEE Trans. Electromagn. Compat., Vol. EMC-28, No. 4, pp. 273-276

November 1986

An Array of Dipoles for Plane Wave Synthesis

Proc., 1985 Intl. Symp. on Antennas and Propagat., 20-22 August

1985, Kyoto, Japan, pp. 177-180 1985

BILL, D. A.; WAIT, J.R.

Propagation Along a Coaxial Cable with a Helical Shield

IEEE Trans. Microwave Theory Tech., Vol. MTT-28, No. 2, pp. 84-89

February 1980

Electromagnetic Characteristics of a Coaxial Cable with Periodic slots IEEE Trans. Electromagn. Compat., Vol. EMC-22,

No. 4, pp. 303-307

November 1980

Anomalous Vertical Magnetic Field for Electromagnetic Induction in a Laterally Varying Thin Conductive sheet

Radio science, Vol. 21, No. 4, pp. 617-621 July-August 1986

BILL, D. A.; CRAWFORD, M. L.; RANDA, M.; WU, D. I.

Aperture Coupling to Shielded Transmission Lines: Theory and Experiment NISTIR 3988

April 1992

HUDSON, P. A.; MELQUIST, D. G.; ONDREJRA, A. R.; WERNER, P. E.

A Program to Evaluate/Improve Instrumentation and Test Methods for

Electroexplosive Device Safety Qualification

NBSIR 73-323

June 1973

Completion of the Program to Evaluate/Improve Instrumentation and Test Methods for Electroexplosive Device Safety Qualification

NBSIR 74-379

June 1974

JESCE, R. I.

Susceptibility of Emergency Vehicle Sirens to External Radiated

Electromagnetic Fields

National Institute of Justice Technology Program, NIJ

Report-200-85

May 1986

A Survey of Triaxial and Mode-stirred Techniques for Measuring the Shielding Effectiveness of Connectors and Cables

NBSIR 86-3060

October $1986^{\circ}$

Measurement of Shielding Effectiveness of Different Cable and Shielding Configurations by Mode-Stirred Techniques

NBSIR $87-3076$

October 1987 
JESCH, R. L. (cont.)

Measurement of Shielding Effectiveness of Different Cable and Shielding Configurations by Mode-Stirred Techniques

IEEE Trans. Electromagn. Compat., Vol.EMC-30, pp. 222-228 August 1988

RANDA, M.

Time and Amplitude Statistics for Electromagnetic Noise in Mines NBSIR 74-378 July 1974

The Characteristics of a Relatively Short Broadband Linear Antenna with Tapered Resistive Loading

Proc., Intl. Symp. Ant. Propagat. Soc., 20-22 June 1977 1977

IEEE Antennas and Propagat. Soc. Dig., 20-22 June 1977, pp. $230-233$

1977

A Relatively Short Cylindrical Broadband Antenna with Tapered Resistive Loading for Picosecond Pulse Measurements

NBSIR 77-861

August 1977

A Relatively Short Cylindrical Broadband Antenna with Tapered Resistive Loading for Picosecond Pulse Measurements

IEEE Trans. Antennas Propagat., Vol. AP-26,

No. 3, pp. 439-447

1977

A Broadband Antenna with Tapered Resistive Loading for EMI Measurements Proc., 1977 IEEE Intl. Symp. on Electromagn. Compat., 2-4 August 1977, Seattle, WA, pp. 13-18 1977

The Characteristics of Broadband, Isotropic Electrical Field and Magnetic Field Probes

NBSIR 77-868

November 1977

Transients in Resistively Loaded Antennas and Their Comparison with Conical Antennas and TEM Horns

NBSIR 78-876

March 1978

Analytical and Numerical Techniques for Analyzing an Electrically Short Dipole with a Nonlinear Load

NBSIR 78-898

November 1978

IEEE Trans. Antennas Propagat., Vol. AP-28, No. 1, pp. 71-78 January 1980

The Characteristics of a Traveling-Wave, Linear Antenna with a Nonlinear Load

Dig. Abstract, 1979 IEEE Ant. Propagat. Soc. Symp. January 1979 
RANDA, M. (cont.)

The Time Domain Characteristics of a Traveling Wave Linear Antenna with

Linear and Non-Linear Loads

NBSIR 78-892(R)

February 1979

The Effects of Resistive Loading on TEM Horns

NBSIR 79-1601

August 1979

IEEE Antennas Propagat., Vol. AP-28, No. 1, pp. 71-78 January 1980

IEEE Trans. Electromagn. Compat., Vol. EMC-24,

No. 2 , pp. 245-255

May 1982

Transients in Resistively Loaded Antennas and Their Comparison with Conical Antennas and a TEM Horn

IEEE Trans. Antennas Propagat., Vol. AP-28,

No. 1, pp. 132-136

January 1980

The Time-Domain Characteristics of a Traveling-Wave Linear Antenna with Linear and Nonlinear Parallel Loads

IEEE Trans. Antennas Propagat., Vol. AP-28, No. 2 March 1980

The Characteristics of a Linear Antenna with Tapered Resistive and Capacitive Loading

Proc., IEEE Ant.Propagat. Soc. Symp., 2-6 June 1980, Quebec,

Canada, Vol. 2, AP. 18-2, pp. 696-699 1980

Theoretical and Experimental Investigations of Loading Effects Due to a Perfectly Conducting Rectangular Cylinder in a Transverse

Electromagnetic Cell

4 th Symp. and Tech. Exhib. on Electromagn. Compat., March 1981,

Zurich, Switzerland 1981

Theoretical and Experimental Investigations of Electromagnetic Field

Distortion Due to a Perfectly Conducting Rectangular Cylinder in a Transverse Electromagnetic Cell

NBS Tech. Note 1028

April 1981

An Electric and Magnetic Field Sensor for Simultaneous Electromagnetic Near-Field Measurements - Theory

NBS Tech. Note 1062

April 1983

An Electromagnetic Near-field sensor for simultaneous Electric and Magnetic-Field Measurements

IEEE Trans. Electromagn. Compat., Vol. EMC-26,

No. 3, pp. 102-110

August 1984

Transients in a Resistively Loaded Loop Antenna

Proc., 1984 Intl. Symp. on Electromagn. Compat.,

16-18 October 1984, Tokyo, Japan, Vol. 1, pp. 286-290 1984 
RANDA, M. (cont.)

A Methodology for Evaluating Microwave Anechoic Chamber Measurements Proc., 1985 Symp. and Technical Exhib. on Electromagn. Compat., 5-7 March 1985, Zurich, Switzerland, pp. 69-74 1985

A Microstrip Patch Antenna as a standard Transmitting and Receiving Antenna.

Dig., 1989 Intl. Symp. on Electromagn. Compat., 8-10 September 1989, Nagoya, Japan, pp. 460-462 1989

IEEE Trans. Electromagn. Compat., Vol. 32 , No. 1 , pp. 5-8 February 1990

RANDA, M.; ADAMS, J. $W$.

Amplitude Statistics of Electromagnetic Noise in Coal Mines

Proc., 1973 Thru-the-Earth Electromagn. Workshop, 15-17 August 1973, Colorado School of Mines, Golden, CO, pp. 156-160 1973

Dig., Intl. Union of Radio Sci. (URSI), IEEE Trans. Antennas Propagat. May 1975

RANDA, M.; DRIVER, L. D.

An Isotropic, Electric-Field Probe with Tapered Resistive Dipoles for Broadband Use, $100 \mathrm{kHz}-18 \mathrm{GHz}$

Record, 1986 IEEE Intl. Symp. on Electromagn. Compat.,

16-18 September 1986, San Diego, CA, pp. 256-261 1986

IEEE Trans. Microwave Theory Tech., Vol. MTT-35,

No. 2 , pp. 124-130

February 1987

A Broadband, Electric-Field Probe Using Resistively Tapered Dipoles, 100 $\mathrm{kHz}-18 \mathrm{GHz}$

Proc., 1986 IEEE/MTT/S Intl. Microwave Symp. Dig., 2-4 June 1986, Baltimore, MD, pp. 621-624

1986

An Optically Linked Electric and Magnetic Field Sensor for Poynting Vector Measurements in the Near Field of Radiating Sources

Dig., Conf. Prec. Electromagn. Meas., 7-10 June 1988, Tsukuba Science City, Japan

pp. $32-33$

$$
1988
$$

RANDA, M.; HILI, D. A.

A Three-Loop Method for Determining the Radiation Characteristics of an Electrically small source

IEEE Trans. Electromagn. Compat., Vol. EMC-34, No. 1, pp. 1-3 February 1992

RANDA, M.; MAY, W. G.

A Millimeter Wave Isolator Containing a Semiconductor Rod in a Circular Waveguide

IEEE Trans. Instrum. Meas., No. IM-24, No. 3, pp. 264-266 September 1975 
RANDA, M.; NAHMAN, N. S.

Standards for Measurement of Electromagnetic Fields

Proc., 1985 Instrum. and Meas. Technol. Conf.,

20-22 March 1985, Tampa, FL, pp. 20-23

1985

RANDA, M.; ORR, R. D.

A Radio-Frequency Power Delivery System: Procedures for Error Analysis and Self-Calibration

NBS Tech. Note 1083

August 1985

Near-Field Gain of a Horn and an Open-Ended Waveguide: Comparison

Between Theory and Experiment

Proc., 5th Intl. Conf. on Electromagn. Compat.

29 September-2 October 1986, University of York, England,

pp. 137-145

1986

Generation of Standard Electromagnetic Fields in a TEM Cell

NBS Tech. Note 1319

August 1988

RANDA, M.; RANDA, J. P.

Estimation of Electromagnetic Fields in Complex Environments

Proc., 9th Intl. Symp. \& Tech. Exhibition, EMC, 12-14 March 1991, Zurich, Switzerland, pp. 337-342 1991

RANDA, M.; RIES, F. $X$.

Time Domain Sensors for Radiated Impulsive Measurements

Proc., IEEE 1982 Intl. Symp. on Electromagn. Compat.,

8-10 September 1982, Santa Clara, CA, pp. 296-301 1982

IEEE Trans. Antennas Propagat., Vol. AP-31, No. 3, pp. $438-444$

May 1983

RANDA, M.; WYSS, J. C.

Evaluation of off-Axis Measurements Performed in an Anechoic Chamber NBS Tech. Note 1305

october 1986

RANDA, M.; ADAMS, J. W.; BENSEMA, W. D.

Electromagnetic Noise in McElroy Mine

NBSIR 74-389

June 1974

RANDA, M.; RANDA, J. P.; NAHMAN, N. S .

Possible Estimation Methodologies for Electromagnetic Field

Distributions in Complex Environments

NBS Tech. Note 1081

March 1985

RANDA, M.; RIES, F. X.; BELSHER, D. R.

A Broadband Isotropic, Real-Time, Electric Field Sensor (BIRES) Using

Resistively Loaded Dipoles

NBSIR 79-1622

December 1979 
RANDA, M.; RIES, F. Z.; DRIVER, L. D.; ORR, R. D.

Design Considerations for Broadband Magnetic-Field Sensors

Dig., 1982 Conf. Prec. Electromagn. Meas., 28 June-1 July 1982,

Boulder, $\mathrm{CO}, \mathrm{pp} . \mathrm{P}-11$ through $\mathrm{P}-13$ 1982

An Electric and Magnetic Field Sensor Concept for simultaneous

Near-Field Electromagnetic Components When Using TEM Cells

Proc., 1983 Electromagn. Compat. Symp. and Exhib.,

8-10 March 1983, Zurich, Switzerland, pp. 263-266 1983

RANDA, M.; LARSEN, E. B.; BORSERO, M.; GAILIANO, P. G.; YODOSHIMA, I.; NAHMAN, N. S.

Standards for Electromagnetic Fields Measurements

Special Issue of Proc. IEEE on Radio Meas. and standards,

Vol. 94, No. 1, pp. 120-128

January 1986

KOEPKE, G. H.; MA, M. T.

A New Method for Determining the Emission Characteristics of an Unknown Interference source

Proc., IEEE 1982 Intl. Symp. on Electromagn. Compat.,

8-10 September 1982, Santa Clara, CA, pp. 151-156 1982

Proc., 1983 Electromagn. Compat. Symp. and Exhib.,

8-10 March 1983, Zurich, Switzerland, pp. 263-266 1983

KOEPKE, G. H.; MA, M. T.; BENSEMA, W. D.

Theory and Measurements of Radiated Emissions Using a TEM Cell

NIST Tech. Note 1326

January 1989

Implementation of an Automated System for Measuring Radiated Emissions Using a TEM cell

IEEE Trans. Instrum. Meas., Vol. IM-38, No. 2, pp. 473-479 April 1989

ROEPKE, G. H.; DRIVER, L. D.; CAVCEY, K. H.; MASTERSON, R. D.; JOBNR, R. T.; KANDA, M.

A standard spherical Dipole source

NIST Tech. Note 1351

December 1991

KUFFEL, J.; MALEWSKI, R.; VAN HEESWIJK, R.; LAWTON, R. A.

Dynamic Performance of Digital Recorders Used for Monitoring

High-Voltage Impulse Tests

Proc., IEEE Instrum. and Meas. Technol. Conf.,

25-27 March 1986, Boulder, CO, Vol. IM-35,

No. 4, pp. 591-595

1986

LARSEN, E. B.

Calibration of Radio Receivers to Measure Broadband Interference

NBSIR 73-335

September 1973 
IAARSEN, E. B. (cont.)

Background and Present Status of RF Probe Development at NBS

Record, "EMC '81," 1981 IEEE Intl. Symp. on Electromagn. Compat., 18-20 August 1981, Boulder, CO 1981

Calibration and Meaning of Antenna Factor and Gain for EMI Antennas Interference Technology Engineers' Master (ITEM 1986), pp. 113-335 1986

LARSEN, E. B.; ANDREWS, J. R.

Using Fiber Optics in A Broadband, Sensitive, Isotropic Antenna -

$15 \mathrm{KHz}$ to $150 \mathrm{MHz}$

Proc., 1976 IEEE Intl. Symp. on Electromagn. Compat., 13-15 July 1976, Washington, DC 1976

LARSEN, E. B.; CRUZ, J. E.

E\&H Fields in Transmission Lines and Coils for susceptibility Testing, Probe Calibration, and RF Exposure Chambers

Proc., 1985 Instrum. and Meas. Technol. Conf., 20-22 March 1985, Tampa, FL, p. 199 1985

LARSEN, E. B.; RIES, F. Z.

Design and Calibration of the NBS Isotropic Electric-Field Monitor $(E F M-5), 0.2$ to $1000 \mathrm{MHz}$

NBS Tech. Note 1033

March 1981

LARSEN, E. B.; SHAFER, J.F.

Surveys of Electromagnetic Field Intensities Near Representative Higher-Power FAA Transmitting Antennas FAA-RD -77

December 1977

IARSEN, E. B.; ANDREWS, J. R.; BALDWIN, E. E.

Sensitive Isotropic Antenna with Fiber-optic Link to a Conventional

Receiver

NBSIR 75-819

September 1976

LARSEN, E. B.; EHRET, R. L.; CAMELI, D. G.; ROEPRE, G. H. Calibration of Antenna Factor at a Ground screen Field Site Using an Automatic Network Analyzer

Proc., IEEE 1989 Natl.Symp. on Electromagn. Compat., 23-25 May 1989, Denver, CO, pp. 19-24 1989

LAWTON, R. A.

A New Standard for Electric Field Strength

IEEE Trans. Instrum. Meas., Vol. IM-19, pp. 45-51 February 1970

LAWTON, R. A.; ONDREJRA, A. R.

Antennas and the Associated Time Domain Range for the Measurement of Impulsive Fields

NBS Tech. Note 1008

November 1978 
LIU, B. H.; CHANG, D. C.; MA, M. T.

Eigenmodes and the Composite quality Factor of a Reverberating Chamber NBS Tech. Note 1066

August 1983

Design Consideration of Reverberating Chambers for Electromagnetic Interference Measurements

Record, IEEE 1983 Intl. Symp. on Electromagn. Compat.,

23-25 August 1983, Arlington, VA, pp. 508-512 1983

MA, M. T.

A Theoretical Study of Unbalanced Ground Effects on Receiving Dipoles NBS IR 79-1605

May 1979

Arrays of Discrete Elements

Antenna Engineering Handbook, Chapter 3, R. C. Johnson and H.

Jasik, McGraw-Hill Book Co.

January 1984

Error Analysis of Radiation Characteristics of an Unknown Interference Source Based on Power Measurements

Proc., 1984 Intl. Symp. on Electromagn. Compat.,

16-18 October 1984, Tokyo, Japan, pp. 39-44 1984

Measurements of Unintentional Electromagnetic Emissions

Proc., IEEE Spec. Issue on Radio Meas. Methods and Standards, Vol. 74, No. 1, pp. 110-111

January 1986

Shielding Effectiveness Measuring Using an Apertured TEM Cell in a Reverberation Chamber

IEEE Intl. Symp. on Electromagn. Compat., August 1986,

San Diego, CA

1986

Characterization of Unknown RF Leakage Sources: Problems, Solutions and Practical Implications

URSI XXIInd General Assembly, 24 August - 2 september 1987,

Tel Aviv, Israel 1987

Understanding Reverberating Chambers as an Alternative Facility for EMC Testing

J. Electromagn. Waves Appl., Vol. 2, pp. 339-351 March \April 1988

Theory and Measurements of Unintentional Radiators

Dig., Conf. Prec. Electromagn. Meas., 7-10 June 1988, Tsukuba

Science City, Japan, pp. 30-31 1988

How High is the Level of Electromagnetic Fields Radiated by an ESD?

Proc., 8th Intl. Symp. \& Tech. Exhibition on EMC,

7-9 March 1989, Zurich, Switzerland, pp. 361-365

1989

EMC Standards and Regulations: A Brief Review

NISTIR 3989

May 1992 
MA, M. T.; ADAMS, J. W.

Phase Characteristics and Time Responses of Unknown Linear Systems

Determined from Measured CW Amplitude Data

NIST Tech. Note 1349

November 1991

System Response to Pulsed Excitations Estimated from Measurement of CW Amplitudes

Proc., Intl. Symp. on Electromagn. Compat., 25-27 May 1992,

Beijing, China, pp. 29-32 1992

MA, M. T.; ARTHUR, M. G.

A Study of Distribution of Electromagnetic Fields Inside Buildings with Apertures Excited by an External source

NBSIR 82-1659

February 1982

MA, M. T.; BENSEMA, W. D.

Automated TEM Cell for Measuring Unintentional EM Emissions

Proc., EMC Expo 1987, Intl. Conf. on Electromagn. Compat.,

19-21 May 1987, San Diego, CA, pp. T11.1 through T11.12 1987

MA, M. T.; CRAWFORD, M. I.

Facilities for Improving Evaluations of Electromagnetic susceptibilities of Weapon systems and Electronic Equipment

NISTIR 89-3928

November 1989

MA, M. T.; FITZGERRELL, R. G.

Design of a Van-Top Low-Profile HF Antenna OTR-77-131

October 1977

MA, M. T.; RANDA, M.

Electromagnetic Compatibility and Interference Metrology

NBS Tech. Note 1099

July 1986

MA, M. T.; ROEPRE, G. H.

A Method to Quantify Radiation Characteristics of an Unknown

Interference source

NBS Tech. Note 1059

October 1982

Uncertainties in Extracting Radiation Parameters for an Unknown

Interference source Based on Power and Phase Measurements

NBS Tech. Note 1064

June 1983

MA, M. T.; LARSEN, E. B.; CRAWFORD, M. L.

Electromagnetic Fields with Arbitrary Wave Impedances Generated Inside a TEM Cell

IEEE Trans. Electromagn. Compat., Vol. EMC-33, No. 4, pp. 358-362 November 1991

MA, M. T.; WILSON, P. F.; CHANG, D. C.

Excitation of a TEM Cell by a Vertical Electric Hertzian Dipole NBS Tech. Note 1037

March 1981 
MA, M. T.; CHANG, D. C.; SREENIVASIAB, I.

A Method of Determining the Emission and Susceptibility Levels of Electrically Small Objects Using a TEM Cell

NBS Tech. Note 1040

April 1981

MA, M. T.; RANDA, M.; CRAWFORD, M. L.; LARSEN, E. B.

A Review of Electromagnetic Compatibility/Interference Measurement Methodologies

Proc., IEEE, Vol. 73, No. 3, pp. 388-411 March 1985

Measuring Electromagnetic Interference, Part I, Open-Field Sites and TEM Cells; Part II, Reverberating Chambers; Part III, Anechoic Chambers and Field Probes

Test and Measurement World, Part I, pp. 72-88, Feb. 1986; Part II, pp. 74-90, May 1986, Part III, pp. 81-99, sept. 1986 1986

MARTZLOFF, F. D.; WILSON, P. F.

Fast Transient Tests - Trivial or Terminal Pursuit?

Proc., 7th Intl. Zurich Symp. and Technical Exhib. on Electromagn. Compat., 3-5 March 1987, Zurich, Switzerland, pp. 283-288 1987

MASTERSON, R. D.

A Photonic Electric Field Probe for Frequencies up to $2 \mathrm{GHz}$

Proc., Soc. for Photo-Optical Instrumentation Engineers (SPIE), Vol. 720 , p. 100 1986

MASTERSON, K. D.: DRIVER, L. D.

A Broadband, Isotropic, Photonic Electric Field Probe for Measurements from $10 \mathrm{kHz}$ to Above $1 \mathrm{GHz}$ Proc., High Bandwith Analog Applications of Photonics II, SPIE Intl. Soc. of Optical Engineers, Vol. 987, 8-9 september 1988, Boston, MA, pp. 107-118 1989

MASTERSON, K. D.; RANDA, M.

Broadband, Photonic Electric Field sensors for EMP and HPM Applications Proc., Fifth National Conf. on High Power Microwave Technology (DoD), $\begin{gathered}10-15 \\ 1990\end{gathered}$ June 1990, West Point, NY, pp. 388-391

MASTERSON, R. D.; DRIVER, L. D.; KANDA, M.

Photonic Probes for the Measurement of Electromagnetic Fields Over Broad Bandwidths

Proc., IEEE 1989 Natl.Symp. on Electromagn. Compat.,

23-25 May 1989, Denver, CO, pp. 1-6 1989

MIILER, C. K. S.

EMI - A Problem of Growing Concern Report of the 63rd Natl. Conf. on Weights and Measures NBS SP 532 1978

Challenges of EMI Measurements Proc., Natl. Conf. of Standards Laboratories, 22-25 september 1980, pp. 8-1 through 8-17 1980 
MILLER, C. R. S. (cont.)

The EMI Measurement Challenge

Proc., 1983 Meas. Sci. Conf., 20-21 January 1983,

Palo Alto, CA, pp. 189-197

1983

NAHMAN, N. S.

Miniature Superconductive Coaxial Transmission Lines

Proc., IEEE, Vol. 61, pp. 76-79

January 1073

NAHMAN, N. S.; RANDA, M.; LARSEN, E. B.; CRAWFORD, M. L.

Methodology for standard Electromagnetic Field Measurements

IEEE Trans. Instrum. Meas., Vol. IM-34, No. 4, pp. 490-503 December 1985

NEWELL, A. C.; CRAWFORD, M. I.

Planar Near-Field Measurements on High Performance Array Antennas NBSIR 74-380

July 1974

ONDREJKA, A. R.; ADAMS, J. W.

Shielding Effectiveness (SE) Measurement Techniques

Proc., 1984 IEEE Natl.Symp. on Electromagn. Compat.,

24-26 April 1984, San Antonio, TX, pp. 249-256 1984

Shielding Effectiveness (SE) Measurement Techniques

Proc., 1984 EMI/RFI Technical Conf., The Soc. of Plastics

Engineers, Inc., 18-20 June 1984, Chicago, IL, pp. 19-26 1984

ONDREJKA, A. R.; RANDA, M.

A Time-Domain Method for Measuring the Reflection Coefficient of Microwave Absorbers at Frequencies Below $1 \mathrm{GHz}$

Dig., 1991 Ant. Prop. Soc. Symp., 24-28 June 1991, London, Ontario, Canada, Vol. 3, pp. 1656-1663 1991

RANDA, J. P.

Theoretical Considerations for a Thermo-Optic Microwave Electric-Field Probe

J. Microwave Power Electromagn. Energy, Vol. 25, No. 3, pp. 133140 1990

Simultaneous vs. Independent Injection Testing of Nonlinear Multiport systems

Proc., 9th Intl. Symp. \& Tech. Exhibition on EMC,

12-14 March 1991, Zurich, Switzerland, pp. 71-74 1991

RANDA, J. P.; RANDA, M.

Multiple-Source, Multiple-Frequency Error of an Electric Field Meter

IEEE Trans. Antennas Propagat., Vol. AP-33, No. 1, pp. 2-9 January 1985

A Directional Scanning Technique for Characterization of Complex Electromagnetic Environments

Dig., 1985 IEEE Ant. Propagat. Soc. Symp., 17-21 June 1985, University of British Columbia, Vancouver, B. C., Canada, pp. $521-524$ 
RANDA, J. P.; RANDA, M.; MELQUIST, D. G.; SEGA, R. M.; NORGARD, J. D. High Frequency Electric Field Probe Development Proc., EMC EXPO '88, Intl. Conf. on Electromagn. Compat., 10-12 May 1988, Washington, DC, pp. T15.31 through T15.37 1988

REEVE, G. R.

Calibration of Impulse Noise Generators

NBSIR 73-343

October 1973

A Standard Percent Modulation Measurement System

Dig. 1976 Conf. Prec. Electromagn. Meas., pp. 165-166 June 1976

Synthesized Isotropic Pattern Antennas for EM Field Measurements Record, "EMC'81," 1981 IEEE Intl. Symp. on Electromagn. Compat., 18-20 August 1981, Boulder, CO 1981

Alternate EMI Measurement Techniques for Microelectronic Circuits Proc., EMC EXPO 1986, Intl. Conf. on Electromagn. Compat., 16-19 June 1986, Washington, DC, pp. T26.1 through T26.4 1986

Proficiency Testing for MIL-STD 462 NVLAP Laboratories Dig., EMC EXPO'88, 10-12 May 1988, Washington, DC, $\mathrm{T} 33.13-\mathrm{T} 33.15$

September 1988

Proficiency Testing for MIL-STD 462 NVLAP Laboratories. Dig., EMC EXPO

'88; 10-12 May 1988; Washington, DC; T33.13 through T33.15. September 1988

REEVE, G. R.; WAINWRIGHT, A. E.

A Frequency Tracking, Tuned, Receiving Monopole Record, IEEE/APS Symp., June 1981 1981

RIES, F. Z.; MILLER, C. K. S .

Influence of Electromagnetic Interference on Electronic Devices Bulletin OIML Seminar December 1981

SCOTT, W. W., Jr.; ADAMS, J. W.; BENSEMA, W. D.; DOBROSKI, H.

Electromagnetic Noise in Lucky Friday Mine NBSIR 74-391

October 1974

SHAFER, J. F.

Field Strength Levels in Vehicles Resulting from Communications

Transmitters

National Institute of Justice Report, 200-83 June 1984

SIMPSON, P. A.

Broadband Pulsed/CW Calibration Signal Standard for Field Intensity

Meter (FIM) Receivers

NBSIR 74-371

June 1974 
RANDA, J. P.; RANDA, M. (cont.)

Directional Scanning of Complex Electromagnetic Environments Proc., 1985 Intl. Symp. on Antennas and Propagat., 20-22 August 1985, Kyoto, Japan, pp. 899-902 1985

A Finite-Element Action Approach to the Characterization of Complex Electromagnetic Environments

Proc., 1985 Intl. Symp. on Antennas and EM Theory, 26-28 August 1985, Beijing, China, pp. 48-53 1985

High Frequency Errors of an Electric-field-meter in Complicated Environments

Record, IEEE 1985 Intl. Symp. on Electromagn. Compat., 20-22 August 1985, Wakefield, MA, pp. 618-621 1985

A Lattice Approach to Complex Electromagnetic Environments Proc., 1986 IEEE Intl. Symp. Electromagn. Compat., September 1986, San Diego, CA, pp. 329-331 1986

A Lattice Approach to Volumes Irradiated by Unknown Sources NBS Tech. Note 1303 October 1986

A Lattice Approach to Environments Irradiated by Unknown Sources Proc., 7th Intl. Electromagn. Compat. Symp., March 1987, Zurich, Switzerland, pp. 191-195 1987

A New Approach to Volumes Irradiated by Unknown Sources IEEE Trans. Electromagn. Compat., pp. 273-281 November 1987

RANDA, J. P.; RANDA, M.; MELQUIST, D. G.

Possible Designs for Electric-Field Strength-Probes for Millimeter Waves NBS.IR 88-3084 February 1988

RANDA, J. P.; RANDA, M.; ORR, R. D.

Thermo-Optic Designs for Electromagnetic Field Probes for Microwaves and Millimeter Waves

IEEE Trans. Electromagn. Compat., Vol. 33, No. 3, pp. 205-314 August 1991

Resistively-Tapered-Dipole Electric-Field Probes up to $40 \mathrm{GHz}$ Proc., IEEE Intl. Electromagn. Compat. Symp. 13-15 August, 1991 Cherry Hill, NJ, pp. 265-266 1991

RANDA, J. P.; RANDA, M.; MELQUIST, D. G.; ORR, R. D. Thermo-optic Designs for Microwave and Millimeter-wave Electric-Field Probes

Proc., IEEE Symp. Electromagn. Compt., 23-25 May 1989,

Denver, CO, pp. 7-11 1989 
SREENIVASIAB, I.; CHANG, D. C.

Variational Expression for the scattering Matrix of a Coaxial Line step

Discontinuity and its Application to an Over Moded Coaxial TEM Cell NBSIR 79-1606

May 1979

SREENIVASIAH, I.; CHANG, D. C.; MA, M. T.

Characterization of Electrically small Radiating sources by Tests Inside

a Transmission Line Cell

NBS Tech. Note 1017

March 1980

A Critical study of Emission and Susceptibility Levels of Electrically Small Objects from Tests Inside a TEM Cell

Record, "EMC '81," 1981 IEEE Intl. Symp. on Electromagn. Compat., 18-20 August 1981, Boulder, CO 1981

Emission Characteristics of Electrically Small Radiating Sources from Tests Inside a TEM Cell

IEEE Trans. Electromagn. Compat., Vol. EMC-23, No. 3, pp. 113-121

August 1981

STUBENRAUCH, C. F.; SPIESS, W.; GALLIANO, P. G.; BABJI, T.

International Intercomparison of Electric Field strength at $100 \mathrm{MHz}$

IEEE Trans. Instrum. Meas., Vol. IM-32, No. 1, pp. 235-237 March 1983

TAGGART, H. E.

Methods of Suppressing Automotive Interference

NBS SP 480-44

November 1981

Radiated EMI Instrumentation Errors

EMC Technology Magazine, Vol. 1, No. 4, pp. 26-35

October 1982

TIPPET, J. C.; CHANG, D. C.

Radiation Characteristics of Dipole Sources Located Inside a

Rectangular, Coaxial Transmission Line

NBSIR 75-829

January 1976

A New Approximation for the Capacitance of a Rectangular, Coaxial, strip Transmission Line

IEEE Trans. Microwave Theory Tech., pp. 602-604

September 1976

Higher Order Modes in Rectangular Coaxial Line with Infinitely Thin

Inner Conductor

NBSIR 78-873

March 1978

Characteristic Impedance of a Rectangular Coaxial Line With offset Inner Conductor

IEEE Trans. Microwave Theory Technol., Vol. MTT-26, No. 11, pp. 876-883

November 1978 
TIPPET, J. C.; CHANG, D. C. (cont.)

Dispersion and Attenuation Characteristics of Modes in a TEM Cell with a Lossy Dielectric Slab

NBSIR 79-1615

July 1979

TIPPET, J. C.; CBANG, D. C.; CRAWFORD, M. I.

An Analytical and Experimental Determination of the Cutoff Frequencies of Higher-Order TE Modes in a TEM Cell NBSIR 76-841

June 1976

VANZURA, E. J.

Automated System for Electromagnetic Field Generation and Immunity Testing

Proc., 1988 IEEE Instrum. Meas. Technol. Conf., 19-22 April 1988, San Diego, CA, pp. 3-10 1988

VANZURA, E. J.; ADAMS, J.W.

Generating Constant Electromagnetic Fields Inside a Partially-Loaded Shielded Room

Test and Meas. World, pp. 72-83

November 1987

WACKER, P. F.

Quantifying Hazardous Microwave Fields: Analysis

Proc., 1969 symp. on Biological Effects and Health Implications of Microwave Radiation, 17-19 September 1969, U.S. Department of Health Education, and Welfare, Rockville, MD. Richmond, VA, BRH/DBE 70-2, pp. 197-203 June 1970

WACKER, P. F.; BOWMAN, R. R.

Quantifying Hazardous Electromagnetic Fields: Scientific Basis and Practical Considerations

IEEE Trans. Microwave Theory Tech., Vol. MTT-19, No. 22 February 1971

WILSON, P. F.

A Comparison Between Near-Field Shielding Effectiveness Measurements

Based on Coaxial Dipoles and on Electrically Small Apertures

IEEE Trans. Electromagn. Compat., Vol. 30, No. 1, pp. 23-28 February 1988

WILSON, P. F.: CHANG, D. C.

Mode Coupling by a Longitudinal slot for a class of Planar Waveguiding structures: Part I - Theory

IEEE Trans. Microwave Theory Tech., Vol. MTT-33,

No. 10 , pp. $981-987$

October 1985

Mode Coupling by a Longitudinal slot for a Class of Planar Waveguiding Structures: Part II - Applications

IEEE Trans. Microwave Theory Tech., Vol. MTT-33, No. 10 , pp. $988-993$

October 1985

WILSON, P. F.; MA, M. T.

Small Aperture Analysis of the Dual TEM Cell

Proc., 1984 IEEE Natl.Symp. on Electromagn. Compat.,

24-26 April 1984, San Antonio, TX, pp. 365-369

1984 
WILSON, P. F.; MA, M. T. (cont.)

Some Problems Associated with Interpreting Shielding Effectiveness Measurement Results

Proc., 1984 EMI/RFI Technical Conf., The Soc. of Plastics Eng.,

Inc., 18-20 June 1984, Chicago, IL, pp. 9-18 1984

Small Aperture Analysis of the Dual TEM Cell and an Investigation of Test object scattering in a single TEM Cell

NBS Tech. Note 1076

October 1984

Small Obstacle Loading in a TEM Cell

Proc., 1984 Intl. Symp. on Electromagn. Compat.,

16-18 October 1984, Tokyo, Japan, pp. 30-35 1984

Input Impedance of a Probe Antenna in a TEM Cell

IEEE Trans. Electromagn. Compat., Vol. EMC-26, No. 4, pp. 154-161

November 1984

Factors Influencing Material Shielding Effectiveness Measurements Record, IEEE 1985 Intl. Symp. on Electromagn. Compat.,

20-22 August 1985, Wakefield, MA, pp. 29-33 1985

Shielding Effectiveness Measurements in a Dual TEM Cell

IEEE Trans. Electromagn. Compat., Vol. EMC-27, No. 3, pp. 137-142

August 1985

Measurements of Electromagnetic Shielding Capability of Materials Proc., IEEE Regional Conf. and Exhib. on Electromagn. Compat., January 1986, Vol. 74, No. 1, pp. 112-115 1986

Electromagnetic Shielding Effectiveness: Measurement Techniques and Interpretations

1986 IEEE Regional Conf. and Exhib. on Electromagn. Compat.,

6 February 1986, Anaheim, CA, pp. 1-14 1986

A study of Techniques for Measuring the Electromagnetic shielding Effectiveness of Materials

NBS Tech. Note 1095

May 1986

Methods for Measuring the Near-Field and Far-Field shielding Effectiveness of Materials

Proc., EMC Expo 1986 Intl. Conf. on Electromagn. Compat., 16-19

June 1986, Washington, DC, pp. \#T28.1-T28.6 1986

Simple Approximate Expressions for Higher-Order Mode Cut off and Resonant Frequencies in TEM Cells

IEEE Trans. Electromagn. Compat., Vol. EMC-28, No. 3, pp. $125-130$

August 1986 
WILSON, P. F.; MA, M. T. (cont.)

Shielding Effectiveness Measurements Using an Apertured TEM Cell in a Reverberation Chamber

Record, 1986 IEEE Intl. Symp. on Electromagn. Compat.,

16-18 September 1986, San Diego, CA, pp. 265-269 1986

Techniques for Measuring the Shielding Effectiveness of Materials Proc., 7th Intl. Sym.p. and Exhib. on Electromagn. Compat., 3-5 March 1987, Zurich, Switzerland, pp. 547-552 1987

Techniques for Measuring the Electromagnetic Shielding Effectivenss of Materials: Part II -- Near-Field Source Simulation

IEEE Trans. on Electromagn. Compat., Vol. 30, No. 3,

pp. 251-259

August 1988

Fields Radiated by Electrostatic Discharges

IEEE Trans. on Electromagnetic Compatibility, vol. 33, No. 1, pp. $10-18$

February 1991

WILSON, P. F.; CHANG, D. C.; MA, M. T.

Input Impedance of a Probe Antenna Exciting a TEM Cell

NBS Tech. Note 1054

April 1982

WILSON, P. F.; MA, M. T.; ADAMS, J. W.

Techniques for Measuring the Electromagnetic Shielding Effectivenss of

Materials: Part I -- Far-Field Source Simulation

IEEE Trans. Electromagn. Compat., Vol. EMC-30, No. 3, pp. 239-250 August 1988

WILSON, P. F.; MA, M. T.; ONDREJRA, A. R.

Fields Radiated by Electrostatic Discharges

Proc., IEEE Intl. Symp. on Electromagn. Compat., 2-4 August 1988, seattle, WA, pp. 179-183 1988

WILSON, P. F.; CHANG, D. C.; MA, M. T.; CRAWFORD, M. L. Theoretical and Experimental Analysis of Coupling Characteristics of Dual TEM Cells

Record, IEEE 1983 Intl. Symp. on Electromagn. Compat.,

23-25 August 1983, Arlington, VA, pp. 513-517 1983

WILSON, P. F.; ONDREJRA, A. R.; MA, M. T.; IAADBURY, J. M. On the Electromagnetic Fields Radiated From Electrostatic Discharges Theory and Experiment

NBS Tech. Note 1314

February 1988

WU, D. I.; CHANG, D. C.

An Investigation of a Ray-Mode Representation of the Green's Function in

a Rectangular Cavity

NBS Tech. Note 1312

September 1987

The Effect of a Large Rotating scatterer in a Rectangular Cavity NBS Tech. Note 1317

March 1988 
WU, D. I. : CHANG, D. C. (cont.)

A Hybrid Representation of the Green's Function in an Over-Moded Rectangular Cavity

IEEE Trans. Microwave Theory and Techniques, Vol. 36,

No. 9, pp. 1334-1342

September 1988

The Effect of an Electrically Large Stirrer in a Mode-Stirred Chamber

IEEE Trans. Electromagn. Compat., Vol. 31, No. 2,

pp. $164-169$

May 1989

WU, D. I.; RANDA, M.

Comparison of Theoretical and Experimental Data for the Near-Field of an Open Ended Rectangular Waveguide.

IEEE Trans. Electromagn. Compat., Vol. EMC-31, No. 4, pp. 353-358. November 1989

WYSS, J. C.; SHEERAN, S.

A Passive, Optical Modulator and Link for Antennas

IEEE J. Lightwave Technol., Vol. LT-3, No. 2, pp. 316-321 April 1985

WYSS, J. C.; ANSON, W. J.; ORR, R. D.

Building Penetration Project

NBSIR 84-3009

September 1984

WYSS, J. C.; KANDA, M.; MELQUIST, D. G.; ONDREJKA, A. R.

Optical Modulator and Link for Broadband Antennas

Dig., 1982 Conf. Prec. Electromagn. Meas.,

28 June-1 July 1982, Boulder, CO, pp. P-16 through P-17 1982 
ADAIR, R. T.

A Precision $30 \mathrm{MHz}$ Waveguide-Below-Cutoff Attenuator with an Absolute Electronic Readout, NBS Model XII

NBSIR 76-833

January 1976

ADAIR, R. T.; LIVINGSTON, E. M.

Coaxial "Intrinsic" Impedance Standards

NIST Tech. Note 1333

october 1989

ADAIR, R. T.; RUSSELI, D. B.

A Calibration Service for $30 \mathrm{MHz}$ Attenuation and Phase shift $S P-250-32$

April 1988

ADAIR, R. T.; REEVE, G. R.; GATTERER, L. E.

Millimeter Wave Standards: An Emerging Need

Proc., IEEE Instrum. and Meas. Techn. Conf., 25-27 March 1986, Boulder, CO, Vol. IM-35, No. 4, pp. 376-382 1986

The Expanding Need for Microwave and Millimeter Wave Calibration Services

NCSL Newsletter, Vol. 27, No. 1, pp. 21-31

January 1987

AGY, D. L.; NELSON, R. E.

An Immittance Transcomparator

1970 Annual Precision Measurement Association Metrology Conf.

(3rd), 17-18 June 1970, Gaithersburg, MD, Vol. 1,

pp. $147-154$

1970

AILRED, C. M.; MANNEY, C. H.

Self-Calibration of Complex Ratio Measuring system

Proc., 1973 Intl. Conf. Meas. and Instrum., 17-23 June 1973,

Dresden, Germany, ACTA IMEKO 1, pp. 157-166 1973

The Calibration and Use of Directional Couplers Without standards IEEE Trans. Instrum. Meas., Vol. IM-25, pp. 84-89 March 1976

BEATTY, R. W.

Impedance Measurements and Standards for Uniconductor Waveguide

Proc., IEEE, Special Issue on Radio Meas. Methods and standards,

Vol. 55, No. 6, pp. 933-941 June 1967

CLAGUE, F. R.

The NIST Automated Coaxial Microwave Power standard

Proc., 1989 Measurement Science Conf., 26-27 January 1989,

Anaheim, $\mathrm{CA}$, pp. 1C-1 through 1C-14 1989

Power Measurement system for $1 \mathrm{mw}$ at $1 \mathrm{GHz}$

NIST Tech. Note 1345

November 1990 
CLAGUE, F. R.; LARSEN, N. T.

A Transient Response Error in Microwave Power Meters Using Thermistor Detectors

28th ARFTG Conf. Dig., 4-5 December 1986, St. Petersburg Beach, FL, pp. 79-89

1986

COOR, C. C.; ALLRED, C. M.

An Excitation System for Piston Attenuators

IEEE Trans. Instrum. Meas., Vol. IM-20, pp. 10-16 February 1971

COUNAS, G. J.: YATES, B. C.

Measurement of Adapter Loss, Mismatch, and Efficiency Using the Dual Six-port

$$
\begin{array}{ll}
\text { NBSIR } & 88-3095 \\
\text { July } 1988
\end{array}
$$

CRAWFORD, M. L.; SMART, G. R.

New Coaxial Thermistor Mounts for use as Precision Transfer Standards Proc., 1970 Instrument Soc. of America Conf., 26-29 October 1970, Philadelphia, PA, N708-70, pp. 1-6 1970

DRIVER, L. D.; RIES, F. X.; REBULDELA, G.

NBS RF Voltage Comparator

NBSIR 78-871

December 1978

EBBESEN, H.; ENGEN, G. F.

Singularities in Calibration of Six-port Network Analyzers

1981 IEEE MTT-S Intl. Microwave Symp. Dig., p. 149 1981

ELIERBRUCH, D. A.

UHF and Microwave Phase-Shift Measurements Proc., IEEE, Special Issue on Radio Meas. Methods and Standards, Vol. 55, No. 6, pp. 960-969 June 1967

ENGEN, G. F.

An Introduction To The Description and Evaluation of Microwave Systems

Using Terminal Invariant Parameters

NBS MN 112

October 1969

A New Method of Characterizing Amplifier Noise Performance

IEEE Trans. Instrum. Meas., Vol. IM-19, pp. 344-349 November 1970

An Extension to the sliding Short Method of Connector and Adaptor Evaluation

NBS JRES, Vol. 75C, pp. 177-183

1971 
ENGEN, G. F. (cont.)

Power Equations: A New Concept in the Description and Evaluation of Microwave systems

IEEE Trans. Instrum. Meas., Vol. IM-20, pp. 49-57

February 1971

Theorem Giving Limits for $S_{22}$ when $S_{11}$ and the Two-Port Efficiency are Known

IEEE Trans. Instrum. Meas., Vol. IM-20, p. 78 February 1971

Theory of UHF and Microwave Measurements Using the Power Equation Concept

NBS Tech. Note 637

April 1973

Mismatch Considerations in Evaluating Amplifier Noise Performance

IEEE Trans. Instrum. Meas., Vol. IM-22, pp. 274-278 september 1973

Calibration of an Arbitrary six-Port Junction for Measurement of Active and Passive Circuit Parameters

IEEE Trans. Instrum. Meas., Vol. IM-22, Pp. 295-299 December 1973

Comments on Practical Analysis of Reflectometers and Power Equation Concepts

IEEE Trans. Instrum. Meas., Vol. IM-23, pp. 104-105 March 1974

An Alternative Calibration Technique for Automated Network Analyzers with Application to Adapter Evaluation

Proc., 1974 IEEE S-MTT Intl. Microwave Symp., 12-14 June 1974, Atlanta, GA, Microwave symposium Digest, pp. 261-262 1974

Calibration Technique for Automated Network Analyzers with Application to Adapter Evaluation IEEE Trans. Microwave Theory Tech. Part II, 1974 symp. Issue, Vol. MTT-22, Pp. 1255-1260. December 1974

Automated Calibration of Directional-Coupler-Bolometer-Mount Assemblies Proc., 1975 IEEE Microwave Theory and Technique symp. Int., 12-14 May, 1975, Palo Alto, CA, pp. 98-99 1975

IEEE Trans. Microwave Theory Tech., Vol. MTT-23, pp. 984-990 December 1975

Measurement of Complex Microwave Circuit Parameters Using Only Power Detectors

Proc., 1976 Conf. Prec. Electromagn. Meas.,

28 June-1 July 1976, Boulder, CO, p. 171

1976 
ENGEN, G. F. (cont.)

Determination of Microwave Phase and Amplitude from Power Measurements

IEEE Trans. Instrum. Meas., Vol. IM-25, pp. 414-418 December 1976

An Improved Circuit for Implementing the Six-Port Technique of Microwave Measurements

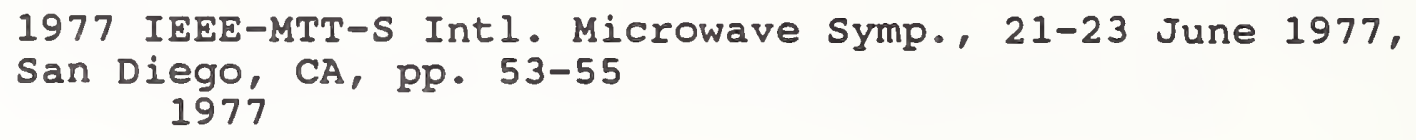

IEEE Trans. Microwàve Theory Tech., Vol. MTT-25, pp. 1080-1083

December 1977

The Six-Port Reflectometer: An Alternative Network Analyzer

Proc., 1977 IEEE-MTT-S Intl. Microwave Symp.,

21-23 June 1977, San Diego, CA, pp. 44-46 1977

Design Considerations for Automatic Network Analyzers Based on the Six-Port Concept

Proc., IEE Euromeas 1977 Conf., 1-9 september 1977, Sussex,

England; London, England, pp. 110-111 1977

Instrumentation: Six Ports simplify Network

Microwave syst. News, pp. 54-55

January 1978

Advances in Microwave Measurement Science

Proc., IEEE, Vol. 66, pp. 374-384

April 1978

The Six-Port Measurement Technique: A Status Report

Microwave J., Vol. 21, pp. 18, 21-22, 24, 84, 87, 89 May 1978

Calibrating the six-port Reflectometer by Means of Sliding Terminations IEEE Trans. Microwave Theory Tech., Vol. MTT-26, pp. 951-957 December 1978

A Least Squares Solution for Use in 6-port Measurement Technique IEEE Trans. Microwave Theory Tech., Vol. MTT-28, No. 12 December 1980

Redundancy: A Monitor of Six-Port Performance

Dig., IEEE 1983 Colloquium on Advances in S-Parameter Measurement at Microwave Lengths, 23 May 1983, Savoy Place, London, England, No. $1983 / 53$, pp. 4-1 through 4-2 1983

In Search of a More Realistic Accuracy Statement for Microwave Metrology Dig., 27th Automatic RF Techniques Group, 5-6 June 1986, Baltimore, $\mathrm{MD}$, pp. 181-183 1986 
ENGEN, G. F. (cont.)

On-Line Accuracy Assessment for the Dual Six-Port ANA: Background and Theory

Dig., 1986 CPEM, 23-27 June 1986, NBS/Gaithersburg, MD, p. 236 1986

IEEE Trans. Instrum. Meas., Vol. IM-36, No. 2, pp. 501-506

June 1987

ENGEN, G. F.; HOER, C. A. Application of an 'Arbitrary' 6-Port Junction to Power Measurement Problems

Proc., 1972 Conf. Prec. Electromagn. Meas., 26-29 June 1972, Boulder, CO, pp. 100-101 1972

IEEE Trans. Instrum. Meas., Vol. IM-21, pp. 470-474 November 1972

Through-Load-Delay: An Improved Technique for Calibrating the Dual Six-port Automatic Network Analyzer

Intl. Microwave Symp. Dig., Orlando, FL, p. 53 1979

ENGEN, G. F.; HUDSON, P. A.

International Intercomparison of Power Standards at $3 \mathrm{GHz}$

IEEE Trans. Microwave Theory Tech., Vol. MTT-19, pp. 411-413 April 1971

ENGEN, G. F.; HOER, C. A.; SPECIALE, R. A.

The Application of "Through-Short-Delay" to the Calibration of the Dual six-port

IEEE Intl. Microwave Symp. Dig., pp. 184-185 1978

ESTIN, A. J.

A Calculable Microwave Attenuation Standard: The High Q Cavity

Proc., 1972 Conf. Prec. Electromagn. Meas.,

26-29 June, Boulder, CO, pp. 100-101 1972

scattering Parameters of SMA Coaxial Connectors

IEEE Trans. Instrum. Meas., Vol. IM-25, No. 4, pp. 329-334 1976

ESTIN, A. J.; JUROSBER, J. R.; MARKS, R. B.; CLAGUE, F. R.; ALLEN, J. W. Basic RF and Microwave Measurements: A Review of selected Programs Metrologia, Vol. 29, No. 2, pp. 135-151 May 1992

FOOTE, W. J.; BUNTER, R. D. Improved Gearing for Rotary-Vane Attenuators

Rev. Scientific Instrum., Vol. 43, No. 7, pp. 1042-1043 July 1972 
FREE, G. M.; JONES, R. N .

Calibration Service for Low-Loss, Three-terminal Capacitance standards at $100 \mathrm{kHz}$ and $1 \mathrm{MHz}$

NIST Tech. Note 1348

February 1992

FURLOW, R。; SHIMODA, R. Y.; WILIIAMS, D. F.; MARKS, R. B.; GUPTA, R. C.

Benchmark for the Verification of Microwave CAD Software

Proc., 38th ARFTG Conf., 5-6 December 1991, San Diego, CA, pp. 97106

1991

GINLEY, R. A.; ALLRED, C. M.

1.25 $\mathrm{MHz}$ Attenuation Measurement System

Proc., IEEE Instrum. and Meas. Techn. Conf., 25-27 March 1986,

Boulder, CO, Vol. IM-35, No. 4, pp. 463-466 1986

HILL, D. A.; WAIT, J.R.

Propagation Along a Coaxial Cable with a Helical Shield

IEEE Trans. Microwave Theory Techn., Vol. MTT-28, No. 2, pp. $84-89$

February 1980

Electromagnetic Theory of the Loosely Braided Coaxial Cable: Part II Numerical Results

IEEE Trans. Microwave Theory Tech., Vol. MTT-28, No. 4,

pp. 326-331

April 1980

BOER, C. A.

The Six-Port Coupler; A New Approach to Measuring V, I, P, Z, and Theta

Dig., 1972 Conf. Prec. Electromagn. Meas.,

26-29 June 1972, Boulder, CO, pp. 6-29

1972

The Six-Port Coupler: A New Approach to Measuring Voltage, Current,

Power, Impedance, and Phase

IEEE Trans. Instrum. Meas., Vol. IM-21, pp. 466-470 November 1972

Using Six-Port and Eight-Port Junctions to Measure Active and Passive Circuit Parameters NBS Tech. Note 673

September 1975

A Microwave Network Analyzer Using Two 6-Port Reflectometers; Proc., 1977 IEEE-MTT-S Intl. Microwave Symp.,

21-23 June 1977, San Diego, CA, pp. 47-49 1977

A Network Analyzer Incorporating Two 6-Port Reflectometers

IEEE Trans. Microwave Theory Tech., Vol. MIT-25,

pp. 1070-1074

December 1977 
BOER, C. A. (cont.)

Calibrating Two Six-Port Reflectometers with only one Impedance standard NBS Tech. Note 1004 June 1978

Calibrating Two Six-Port Reflectometers With an Unknown Length of Precision Transmission Line

Proc., 1978 IEEE MTT-S Intl. Microwave Symp. Dig.,

27-29 June 1978, Ottawa, Canada, pp. 176-178 1978

Calibrating a six-Port Reflectometer with Four Impedance Standards NBS Tech. Note 1012 March 1979

Performance of a Dual Six-Port Automatic Network Analyzer

Proc., IEEE MTT-S 1979, Intl. Microwave Symp. Dig.: The world of Microwaves, 3 April-2 May 1979, Orlando, FL, IEEE Cat. No.

79CHI 439-9 MTT-S 1979

A High Power Dual Six-port Automatic Network Analyzer Used in

Determining Biological Effects of $R F$ and Microwave Radiation

IEEE Trans. Microwave Theory Tech., Vol. MTT-29

December 1981

Choosing Line Lengths for Calibrating Network Analyzers

IEEE, Trans. Microwave Theory Tech., Vol. 31, No. 2, pp. 76-78 January 1983

Multiport Network Analyzers

MCGraw-Hill Yearbook of Science and Technol., pp. 289-292 1986

On-Line Accuracy Assessment for the Dual Six-Port ANA; Treatment of

Systematic Errors

Dig., 1986 CPEM, 23-27 June 1986, NBS/Gaithersburg, MD,

pp. $238-239$

1986

IEEE Trans. on Instrum. and Meas., Vol. IM-36, No. 2, pp. $514-519$

June 1987

Some Questions and Answers Concerning Air Lines as Impedance Standards. Dig., 29th Automatic RF Techniques Group; 12-13 June 1987; Las Vegas, NV; pp. 161-173 1987

An Equivalent Circuit for Imperfect Transmission Line Connectors Dig., Conf. Prec. Electromagn. Meas., 7-10 June 1988, Tsukuba Science City, Japan,

pp. $264-265$

1988

Systematic Errors in Power Measurements Made with a Dual Six Port ANA; NIST Tech. Note 1332

July 1989 
HOER, C. A.; AGY, D. I.

Broad-Band Resistive-Divider-Type Directional Coupler

IEEE Trans. Instrum. Meas., Vol. IM-19, pp. 336-343 November 1970

HOER, C. A.; ENGEN, G. F.

Analysis of a Six. 'nrt Junction for Measuring, V, I, A, B, Z, Gamma, and Phase

Proc., 1973 Conf. of the Intl. Meas. Confederation (6th), 17-23 June 1973, Dresden, Germany, Acta Imeko, Vol. 1, pp. $213-222$

1973

Calibrating a Dual Six-Port or Four-Port for Measuring Two-Ports with Any Connectors

Dig., 1986 IEEE Microwave Theory Tech., Symp., 2-4 June 1986,

Baltimore, MD,

pp. $665-668$

1986

On-Line Accuracy Assessment for the Dual Six-Port ANA: Extension to Non-Mating Connectors

Dig., 1986 Conf. Prec. Electromagn. Meas., 23-27 June 1986,

NBS/Gaithersburg, MD,

pp. 241-242

1986

IEEE Trans. Instrum. Meas., Vol. IM-36, No. 2,

pp. 524-529

June 1987

BOER, C. A.; ROE, R. C.

Using an Arbitrary Six-Port Junction to Measure Complex Voltage Ratios IEEE Trans. Microwave Theory Tech., Vol. MTT-23, No. 12,

pp. $978-984$

December 1975

BOER, C. A.; ROE, R. C.; ALLRED, C. M.

Measuring and Minimizing Diode Detector NonLinearity

Proc., 1976 Conf. Prec. Electromagn. Meas., 28 June-1 July 1976, Boulder, CO, pp. 108-109 1976

BOLT, D. R.

Determination of Scattering Parameters from Precision Coaxial Air-Line Standards

Dig., Conf. Prec. Electromagn. Meas., 7-10 June 1988, Tsukuba Science City, Japan, p. 263 1988

Scattering Parameters Representing Imperfections in Precision Coaxial

Air Lines

NIST JRES, Vol. 94, No. 2, pp. 117-133

March-April 1989 
HOLT, D. R. (cont.)

Determination of scattering Parameters with Respect to thecharacteristic Impedance of Precision Coaxial Air-Line Standards

Dig., Conf. Prec. Electromagn. Meas., 11-14 June 1990, Ottawa, Canada, pp. 282-283

June 1990

HOLT, D. R.; BOER, C. A.

Estimation of True Power Ratios in Six-port Network Analyzers Using Diode Detectors

Proc., 1985 Instrum. and Meas. Technol. Conf., 20-22 March 1985, Tampa, FL, pp. 140-141 1985

IEEE Trans. Instrum. Meas., Vol. IM-34, No. 4, pp. 558-563

December 1985

BUDSON, P. A.

Measurement of RF Peak Pulse Power

Proc., IEEE, Special Issue on Radio Meas. Methods and Standards, Vol. 55, No. 6, pp. 851-855 June 1967

The National Measurement system for Medical Ultrasonics NBSIR 75-937 February 1976

BUDSON, P. A.; SAULSBERY, L. F. An Adjustable-slot-Length UHF Coaxial Coupler with Decade Bandwidth IEEE Trans. Microwave Theory Tech., Vol. MTT-19, pp. 781-783 September 1971

BUDSON, P. A.; MELQUIST, D. G.; ONDREJRA, A. R.; WERNER, P. E. Completion of the Program to Evaluate/Improve Instrumentation and Test Methods for Electroexplosive Device safety Qualification NBSIR 74-379 June 1974

HUNILEY, L. E.; JONES, R. N . Lumped Parameter Impedance Measurements Proc., IEEE, Special Issue on Radio Meas. Methods and standards, Vol. 55 , No. 6 , pp. 900-911 June 1967

JESCH, R. I.

Impedance Measurements in Coaxial Waveguide Systems

Proc., IEEE, Special Issue on Radio Meas. Methods and Standards, Vol. 55, No. 6, pp. 912-923 June 1967

Repeatability of SMA Coaxial Connectors

IEEE Trans. Instrum. Meas., Vol. IM-25, No. 4, pp. 314-320 December 1976 
JESCB, R. I. (cont.)

Evaluation of Low-Loss/Low-Reflection Two-Port Devices or Adapters by Automated Measurement Techniques NBSIR 78-870

December 1977

JESCH, R. I.; HOER, C. A.

Characterization of a High Frequency Probe Assembly for Integrated Circuit Measurements

NBS Tech. Note 663
January 1975

JONES, R. N.

The Measurement of Lumped Parameter Impedance

NBS MN 141

June 1974

Evaluation of Three-Terminal and Four-Terminal Pair Capacitors at High Frequencies

NBS Tech. Note 1024

September 1980

A Comparison of Centrifuge and Freezing Calorimeter Methods for Measuring Free Water in snow

NBSIR 79-1604

April 1979

JONES, R. N.; HUNTLEY, L. E.

A Precision, High Frequency Calibration Facility for Coaxial Capacitance Standards

NBS Tech. Note 386

March 1970

JUDISH, R. M.

Quality Control of Measurements--Measurement Assurance

Proc., IEEE Spec. Issue on Radio Meas. Methods and Standards, Vol. 74, No. 1, pp. 23-25

January 1986

On-Line Accuracy Assessment for the Dual Six-Port ANA: Statistical Methods for Random Errors

Dig., 1986 CPEM, 23-27 June 1986, NBS/Gaithersburg, MD, p. 237

1986

JUDISE, R. M.; BURNS, J. G.

Measurement Program Compares Automatic Vector Analyzers

Microwaves and RF, pp. 203-206

May 1991

JUDISH, R. M.; ENGEN, G. F .

On-Line Accuracy Assessment for the Dual Six-Port ANA: Statistical

Methods for Random Errors

IEEE Trans. Instrum. Meas., Vol. IM-36, No. 2,

pp. 507-513

June 1987 
JUDISH, R. M.; JONES, R. N.

A Generalized Method for the Calibration of Four-Terminal-Pair Type

Digital Impedance Meters

NBSIR 84-3016

August 1984

JUROSHER, J.R.

On-Line Accuracy Assessment for the Dual Six-Port ANA: Experimental

Results

Dig., 1986 Conf. Prec. Electromagn. Meas., 23-27 June 1986, NBS/Gaithersburg, MD, p. 240 1986

IEEE Trans. Instrum. Meas., Vol. IM-36, No. 2, pp. $520-523$ June 1987

A Study of Measurement of Connector Repeatability Using Highly Reflecting Loads

IEEE Trans. Microwave Theory Tech., Vol. MTT-35, No.4, pp. 457-460 April 1987

JUROSHER, J. R.; HOER, C. A.

A Dual Six-port Network Analyzer Using Diode Detectors

IEEE Trans. Microwave Theory Tech., Vol. MTT-32, No. 1, pp. $78-82$

January 1984

A High-Power Automatic Network Analyzer for Measuring the RF Power Absorbed by Biological Samples in a TEM Cell

IEEE Trans. Microwave Theory Tech., Vol. MTT-32, No. 8, pp. 818-824

August 1984

A Technique for Extending the Dynamic Range of the Dual six-port Network Analyzer

IEEE Trans. on Microwave Theory and Tech., Vol. MTT-33, No. 6, pp. 453-459

June 1985

JUROSHEK, J.R.; HOER, C. A.; RAISER, R. R. F.

Calibrating Network Analyzers with Imperfect Test Ports

IEEE Trans. Instrum. Meas., Vol. 38, No. 4,

pp. 898-901

August 1989

RAMPER, R. A.

The National Electromagnetic Measurement system

NBSIR 75-936

June 1977

Status of RF and Microwave Calibration Services at NBS

Natl. Conf. of Standards Laboratories Newsletter, Vol. 18,

No. 3 , p. 40

December 1978 
KAMPER, R. A. (cont.)

Current Trends in NBS Calibration Services

NCSL Newsletter, Vol. 22, No. 1, pp. 38-39

March 1982

RAMPER, R. A.; HOER, C. A.

Millimeter Wave Standards at the National Bureau of Standards (NBS)

Proc., The Intl. Soc. for Optical Engineering (SPIE),

23-24 August 1983, San Diego, CA, Vol. 423, pp. 144-146 1983

RANDA, M.; MAY, W. G.

A Millimeter Wave Reflection Beam Isolator

IEEE Trans. Microwave Theory Tech., Vol. MTT-23, No. 6, pp. 506-508

June 1975

RERNS, D. M.

Definitions of $v, i, Z, Y, a, b, \Gamma$, and $S$

Proc., IEEE, Special Issue on Radio Meas. Methods and Standards, Vol. 55, No. 6, pp. 892-900 June 1967

KOMARER, E. L .

An Application of the Power Equation Concept and Automation Techniques

to Precision Bolometer Unit Calibration

Proc., 1974 IEEE S-MTT Intl. Microwave Symp., 12-14 June 1974,

Atlanta, GA, Microwave Symposium Digest, pp. 263-265 1974

An Automated Broadband System for Measurement of One-Port Microwave Parameters

Proc., 1976 Conf. Prec. Electromagn. Meas., 28 June-1 July 1976, Boulder, CO, pp. 167-170 1976

An Application of the Six-Port Junction to Precision Measurement of Microwave One-Port Parameters

Proc., 1977 IEEE-MTT-S Intl. Microwave Symp., 21-23 June 1977, San

Diego, CA, pp. 56-57 1977

Performance Characteristics of an Automated Broad-Band Bolometer Unit Calibration system

IEEE Trans. Microwave Theory, Vol. MTT-25, pp. 1122-1127

December 1977

Advantages of the Six-port Reflectometer for RF/Microwave Power Measurement in Operational systems

Government Micro-Circuit Applications Conf. Dig. 1978 
ROMARER, E. L.; TRYON, P. V.

An Application of the Power Equation Concept and Automation Techniques to Precision Bolometer Unit Calibration

IEEE Trans. Microwave Theory Tech. Part II, 1974 Symp. Issue, Vol. MTT-22, Pp. 1260-1267

December 1974

IARSEN, N. T.

A New Self-Balancing DC-Substitution RF Power Meter

Proc., 1976 Conf. Prec. Electromagn. Meas., 28 June-1 July 1976, Boulder, CO, pp. 203-205 1976

IEEE Trans. Instrum. Meas., Vol. IM-25, pp. 343-347 December 1976

NBS Type IV RF Power Meter Operation and Maintenance NBSIR 77-866 October 1977

Microwave Power Standards at the National Bureau of standards 1987 NCSL Workshop and Symp., 12-16 July 1987, Denver, CO, pp. 34-1 through 34-7 1987

IARSON, W.; CAMPBELL, E.

Microwave Attenuation Measurement system (Series substitution) NBS Tech. Note 647 February 1974

LAWTON, R. A.; ALLRED, C. M.; HUDSON, P. A.

A Wide Range CW Power Measurement Technique

IEEE Trans. Instrum. Meas., Vol. IM-19, pp. 28-34 February 1970

IITTLE, W. E.; YATES, B. C.

Reflection Coefficient Standards for Automated Network Analyzers Proc., 1976 Conf. Prec. Electromagn. Meas., 28 June-1 July 1976, Boulder, CO, pp. 128-129 1976

LITTLE, W. E.; WAREFIELD, J. P.; HEIM, L.; ALLRED, C. M.; ZAPF, T. I. An NBS Developed Automatic Network Analyzer Proc., 1976 Conf. Prec. Electromagn. Meas., 28 June-1 July 1976, Boulder, $\mathrm{CO}$, pp. 382-385 1976

LIVINGSTON, E. M.; ADAIR, R. T. Performance Evaluation of Radiofrequency, Microwave and Millimeter Wave Power Meters

NBS Tech. Note 1310

December 1988 
MAJOR, J.R.; LIVINGSTON, E. M.; ADAIR, R. T.

Automatic Frequency Response of Frequency Modulated Generators Using the Bessel Null Method

Dig., 24th Automatic RF Techniques Group Conf., 5-6 December 1984, Columbia, MD, pp. 131-153 1984

Proc., 1985 Test and Measurement World Expo, 14-16 May 1985, San Jose, CA, pp. 78-100 1985

NBS Tech. Note 1093

March 1986

MARRS, R. B.

Comments on "Improved Calibration and Measurement of the Scattering Parameters of Microwave Integrated Circuits"

IEEE Trans. on Microwave Theory Tech., Vol. 38, No. 4, p.453 April 1990

Multi-Line Calibration for MMIC Measurement

Digest, 36th ARFTG Conf., 29-30 Nov. 1990, Monterey, CA, pp. 47-55 November 1990

A Multi-Line Method of Network Analyzer Calibration

IEEE Trans. on Microwave Theory Tech., Vol. 39, No. 7, p. 12051215

July 1991

Comments on "Rapid Pulsed Microwave Propagation"

IEEE Microwave and Guided Wave Letters, Vol. 2, No. 5, p. 204 May 1992

MARKS, R. B.; PHILLIPS, R. R.

Wafer-Level ANA Calibrations at NIST

Digest, 34 th ARFTG Conf., 30 Nov. - 1 Dec. 1989, Ft. Lauderdale, FL, pp. 11-25

December 1989

MARKS, R. B.; WILLIAMS, D. F.

Characteristic Impedance Determination Using Propagation Constant Measurement

IEEE Microwave and Guided Wave Letters, Vol. 1, No. 6, p. 141-143 June 1991

Reciprocity Relations for On-Wafer Power Measurements

Proc., 38th ARFTG Conf., 5-6 December 1991, San Diego, CA, pp. 82-89

December 1991

NABMAN, N. $\mathbf{s}$.

A Note on the Transition (Rise) Time Versus Line Length in Coaxial Cables

IEEE Trans. Circuit Theory, Vol. CT-20, pp. 165-167 March 1973 
ONDREJKA, A. R.

Peak Pulse Voltage Measurement (Baseband Pulse)

Proc., IEEE, Special Issue on Radio Meas. Methods and Standards, Vol. 55, No. 6 , pp. 882-885 June 1967

PHILIIPS, R. R.; WILLIAMS, D. F。

MMIC Package Characterization with Active Loads

Digest, 36th ARFTG Conf., 29-30 Nov. 1990, Monterey, CA, pp. 64-72 November 1990

REEVE, G. R.

Millimeter Wave Metrology at the National Institute of standards and Technology

PrOC., 1991 NCSL Workshop \& Symp., 18-22 Aug. 1991, Albuquerque, NM, pp. 183-195

August 1991

REEVE, G. R.; ARTHUR, M. G.

A Standard for RF Modulation Factors

NBS Tech. Note 1016

September 1979

REEVE, G. R.; MILIER, C. R. S .

Current NBS Metrology Capabilities and Limitations at Millimeter Wave Frequencies

Proc., 1985 Meas. Science Conf., 17-18 January 1985,

Santa Clara, CA, pp. 296-314

1985

REEVE, G. R.; MARKS, R. B.; BLACKBURN, D.

MMIC Related Metrology at the National Institute of standards and Technology.

Record, Instrum. Meas. Tech. (IMTC) Conf.; 13-15 February 1990;

San Jose, CA; pp. 196-199. 1990

Microwave Monolithic Integrated Circuit-Related Metrology at the National Institute of standards and Technology

IEEE Trans. Instrum. Meas., Vol. IM-39, No. 6, pp. 958-961 December 1990

RIES, F. $\mathbf{X}$.

An International Intercomparison of Voltage standards at $1 \mathrm{GHz}$ in Coaxial Transmission Line

IEEE Trans. on Instrum. and Meas., Vol. IM-25, p 4. September 1976

ROE, K. C.; HOER, C. A.

A Microwave Vector Voltmeter system

NBSIR 76-844

August 1976

RUMFELT, A. Y ; ELWELI, L. B.

Radio Frequency Power Measurements

Proc., IEEE, Special Issue on Radio Meas. Methods and Standards, Vol. 55, No. 6, pp. 837-850

June 1967 
RUSSELL, D. H.

An Unmodulated Twin-Channel Microwave Attenuation Measurement System; ISA Trans., Vol. 4, pp. 162-169 April 1965

RUSSELL, D. H.; LARSON, W.

RF Attenuation

Proc., IEEE, Special Issue on Radio Meas. Methods and Standards, Vol. 55, No. 6, pp. 942-959

June 1967

SAULSBERY, L. F.; ADAIR, R. T.

ANA Measurement Results on the ARFTG Traveling Experiment

Conf. Dig., 28th Automatic RF Techniques Group, 4-5 December 1986, St. Petersburg Beach, FL, pp. 65-78

$$
1987
$$

SCOTT, W. W., Jr.

A New Coaxial RF-DC Ammeter

Proc., 1970 Conf. Prec. Electromagn. Meas., 2-5 June 1970,

Boulder, CO, Special Issue CPEM Digest p. 13 1970

SCOTT, W. W., Jr.; FREDERICK, N. V.

The Measurement of Current at Radio Frequencies

Proc., IEEE, Special Issue on Radio Meas. Methods and Standards,

Vol. 55, No. 6, pp. 886-891

June 1967

SELBY, M. C.

Voltage Measurement at High and Microwave Frequencies in Coaxial Systems Proc., IEEE, Special Issue on Radio Meas. Methods and Standards, Vol. 55, No. 6, pp. 877-882

June 1967

SEQUEIRA, H. B.; YATES, B. C.

Approach for Evaluating Effects of Wall Losses on Quarter-Wave Losses on Quarter-Wave Short-Circuit Impedance Standards

IEEE Trans. Microwave Theory Tech., Vol. MTT-33,

No. 11, pp. 1106-1109

November 1985

SHERWOOD, G. V.

Air Gage Size Measurement of Microwave Standards

Proc., 1991 Measurement Science Conf. 31 Jan. - 1 Feb. 1991,

Anaheim, CA, pp. 1-10 1991

SIMPSON, P. A.

Broadband Pulsed/CW Calibration Signal Standard for Field Intensity

Meter (FIM) Receivers

NBSIR 74-371

June 1974

SIMPSON, P. A.; HUDSON, P. A.

Implementation of the Notch Technique as an RF Peak Pulse Power Standard NBS Tech. Note 682

July 1976 
SIMPSON, P. A.; ONDREJRA, A. R.

Improvements to the NBS RF Peak-Pulse Power standard 1970 Instrument Soc. of America Silver Jubilee Intl. Conf. and Exhibit, October 1970, Philadelphia, PA, Paper 709-70, pp. 1-7 1970

SLADER, M. J.; JESCH, R. L.

Standardization of Coaxial Connections in the IEC Proc., IEEE Spec. Issue on Radio Meas. Methods and Standards, pp. $14-18$

January 1986

WEIDMAN, M. P.

A Semi-Automated Six-Port for Measuring Millimeter Wave Power and Complex Reflection Coefficient

Proc., 1977 IEEE-MTT-S Intl. Microwave Symp., 21-23 June 1977, San Diego, CA, pp. 58-60 1977

IEEE Trans. Microwave Theory Technol., Vol. MTT-25, pp. 1083-1085

December 1977

WR-10 Single six-port Measurement system

NBSIR 81-1650

September 1981

Finline Diode Six-Port: Fundamentals and Design Information

NBS Tech. Note 1090

December 1985

WEIDMAN, M. P.; CAMPBELI, E.

A Method for Designing Multi-screw Waveguide Tuners

NBS Tech. Note 393

October 1970

WEIDMAN, M. P.; ENGEN, G. F.

Application of a Non-Ideal sliding short to Two-Port Loss Measurement NBS Tech. Note 644

October 1973

WEIDMAN, M. P.; HUDSON, P. A.

WR-10 Millimeter Wave Microcalorimeter

NBS Tech. Note 1044

June 1981

WEIL, C. M.; MARLER, F. E.; MAJOR, J.R.; WEIDMAN, M. P.;

RUSSELI, D. H.

Dual Six-Port Reflectometer Systems Using Waveguide in the Frequency

Range 18-50 GHz.

Digest, 33rd ARFTG Conf., 15-16 June 1989, Long Beach, CA

pp. 76-87.

June 1989 
WILIIAMS, D. F.

Development of On-Wafer Microwave standards at NIST

Digest, 34th ARFTG Conf., 30 Nov. - 1 Dec. 1989, Ft. Lauderdale, FL, pp. 5-10. December 1989

WILIIAMS, D. F.; MARKS, R. B.

The Interpretation and Use of $\mathrm{S}$-Parameters in Lossy Lines Digest, 36th ARFTG Conf., 29-30 Nov. 1990, Monterey, CA, pp. 84-89 November 1990

Transmission Line Capacitance Measurement

IEEE Microwave and Guided Wave Letters, Vol. 1, No. 9, pp. 243-245 September 1991

Frequency-Dependent Transmission Iine Parameters

Digest, IEEE Topical Meeting on Electrical performance of

Electronic Packaging, 22-24 April 1992, Tucson, AZ, pp. 125-127 April 1992

WIILIAMS, D. F.; MARKS, R. B.; DAVIDSON, A.

Comparison of On-Wafer Calibrations

Proc., 38th ARFTG Conf., 5-6 December 1991, San Diego, CA, pp. $68-81$

December 1991

WILLIAMS, D. F.; MARKS, R. B.; PHILLIPS, R. R.

Translate LRL and LRM Calibrations

Microwaves \& $\mathrm{RF}$, pp. 78-84

February 1991

WIILIAMS, D. F.; MARKS, R. B.; PHIIIIPS, R. R.; MIERS, T.

Progress Toward MMIC On-Wafer Standards

Digest, 36th ARFTG Conf., 29-30 Nov. 1990, Monterey, CA, pp. 73-83 November 1990

YATES, B. C.; COUNAS, G. J.

Summary of WR15 Flange Evaluation at $60 \mathrm{GHz}$

NBS Tech. Note 642

October 1973

YATES, B. C.; LARSON, W.

Millimeter Attenuation and Reflection Coefficient Measurement System NBS Tech. Note 619

July 1972 
ARTHUR, M. G.

A Precision HF-Noise Power Measurement system

Instr. Soc. Amer. ISA-70 Conf., October, 1971 1971

The Measurement of Noise Performance Factors: A Metrology Guide NBS MN 142

June 1974

BOYLE, D. R.; CLAGUE, F. R.; REEVE, G. R.; WAIT, D. F.; KANDA, M.

An Automated Precision Noise Figure Measurement system at $60 \mathrm{GHz}$

IEEE Trans. Instrum. Meas., Vol. IM-21, No. 4, pp. 543-549 November 1972

COUNAS, G. J.

NBS $2.0 \mathrm{GHz}$ to $4.0 \mathrm{GHz}$ Automated Radiometer Operation and Service Manual

NBSIR 84-1697

January 1984

COUNAS, G. J.; BREMER, T. B.

NBS $30 / 60$ Megahertz Noise Measurement system Operation and Service Manual

NBSIR 81-1656

December 1981

DAYWITT, W. C.

A Reference Noise standard for Millimeter Waves

IEEE Trans. Microwave Theory Technol., Vol. MTT-21, No. 12, pp. 845-847

December 1973

Error Equations Used in the NBS Precision G/T Measurement System NBSIR 76-842

September 1976

A Precision Earth-Terminal system for Accurate C/KT, G/T, and EIRP Measurements with a Calibrated Radio star

Proc., 1977 URSI Intl. Symp. on Meas. in Telecommunications, 3-7 October, 1977, Lannion, France, pp. 1-4

1977

Error Equations Used in the NBS Earth-Terminal Measurement system NBSIR 78-869

December 1977

Atmospheric Propagation Equations Used in the NBS Earth Terminal

Measurement system

NBSIR 78-883

Apri1 1978

An Error Analysis for the Measurement of Satellite EIRP using a Calibrated Radio star

IEEE Trans. Instrum. Meas., Vol. IM-27, No. 3, pp. 253-258 September 1978

Design and Error Analysis for the WR10 Thermal Noise standard NBS Tech. Note 1071

December 1983 
DAYWITI, W. C. (cont.)

An Error Analysis for the Use of Presently Available Lunar Radio Flux Data in Broadbeam Antenna-system Measurements NBS Tech. Note 1073 February 1984

Proc., 1984 Antenna Applications Symp., 19-21 September, 1984, University of Illinois, Urbana-Champaign, IL, pp. 1-26 1984

A Coaxial Noise standard for the $1 \mathrm{GHz}$ to $12.4 \mathrm{GHz}$ Frequency Range NBS Tech. Note 1074 March 1984

A Preliminary Investigation into using the sun as a Source for G/T Measurements

NBSIR 84-3015

August 1984

A Derivation for the Noise Temperature of a Horn-type Noise Standard Intl. J. Sci. Metrol., Metrologia, 21 pp. 127-133 1985

Broadband Noise Source Applications

Proc., 1985 Instrum. and Meas. Technol. Conf.,

20-22 March, 1985, Tampa, FL, pp. 165-166 1985

10-60 GHz G/T Measurements Using the Sun as a Source--A Preliminary study

NBSIR $86-3046$

April 1986

A Simple Technique for Determining Joint Losses on a Coaxial Iine from Swept-Frequency Reflection Data

Dig., 1986 Conf. Prec. Electromagn. Meas., 23-27 June, 1986, NBS/Gaithersburg, MD, p. 40 1986

IEEE Trans. Instrum. Meas., Vol. IM-36, pp. 468-473 June 1987

Complex Admittance of a Lossy Coaxial Open Circuit with a Hollow Center Conductor

Intl. J. of Sci. Metrol., Metrologia, 24, pp. 13-22 1987

A Simple Technique for Investigating Defects in Coaxial Connectors IEEE Trans. Microwave Theory Tech., Vol. MTT-35,

No. 4, pp. 460-464

April 1987

Radiometer Equation and Analysis of systematic Errors for the NIST Automated Radiometers

NIST Tech. Note 1327

March 1989

First Order Principal Mode Fields and Distributed Line Parameters for a Slightly Lossy Coaxial Transmission Line.

Digest, Conf.Prec. Electromagn. Meas., 11-14 June 1990, Ottawa,

Canada, pp. 404-405

June 1990 
DAYWITT, W. C. (cont.)

First Order Symmetric Modes for a slightly Lossy Coaxial Transmission

Line

IEEE Trans. Microwave Theory Tech., Vol. 38, no. 11. pp.1644-1650 November 1990

Exact Principal Mode Field for a Lossy Coaxial Line

IEEE Trans. Microwave Theory Tech., Vol. 39, No. 8, pp. 1313-1322 August 1991

DAYWITT, W. C.: COUNAS, G. J.

Measuring Adapter Efficiency Using a sliding short circuit.

IEEE Trans. Microwave Theory Tech., Vol. 38, No. 3, pp. 231-237 March 1990

DAYWITI, W. C.; RANDA, M.

G/T Measurement Errors with Radio Stars

Proc., 1975 IEEE Intl. Antennas and Propagat. Symp.,

2-4 June, 1975, Urbana-Champaign, IL, Session 20, pp. 460-463 1975

DAYWITT, W. C.; FOOTE, W. J.; CAMPBEIL, E.

WR15 Thermal Noise standard

NBS Tech. Note 615

March 1972

ENGEN, G. F.

A New Method of Characterizing Amplifier Noise Performance

IEEE Trans. Instrum. Meas., Vol. IM-19, pp. 344-349 November 1970

Mismatch Considerations in Evaluating Amplifier Noise Performance

IEEE Trans. Instrum. Meas., Vol. IM-22, pp. 274-278 September 1973

ESTIN, A. J.; DAYWITT, W. C.

Evaluation of Signal Plus Noise Detection Error in an Envelope Detector with Logarithmic Compression

Proc., 1978 Conf. Prec. Electromagn. Meas., 26-29 June, 1978,

Ottawa, Canada 1978

NBSIR 79-1614(R)

August 1979

IEEE Trans. Inf. Theory, Vol. IT-17, No. 5, pp. 663-664 September 1981

GEYER, R. G.

Magnetostatic Measurements for Mine Detection

NISTIR 88-3098

October 1988

BAIFORD, D.

Infrared-Microwave Frequency Synthesis Design: Some Relevant Conceptual

Noise Aspects

Proc., 1971 Frequency Standards and Metrology Seminar,

30 August - 1 September, Quebec, Canada, pp. 431-466 1972

Comparing Frequencies

Physics Today, Vol. 26, No. 2, p. 15

February 1973 
HALFORD, D. (cont.)

Transparent Metrology of Signal to Noise Ratios of Noisy Band-limited

Digital Signals

NBS Tech. Note 1077 June 1985

RANDA, M.

A Measure for the Stability of Solid state Noise Sources

Proc., 1975 IEEE Intl. Microwave Theory and Technique Symp.,

12-14 May, 1975, Palo Alto, CA, pp. 315-317 1975

Study of Errors in Absolute Flux Density Measurements of Cassiopeia A NBSIR 75-822

October 1975

IEEE Trans. Instrum. Meas., Vol. IM-25, No. 3, pp. 173-182 September 1976

An Improved Solid State Noise Source

Proc., 1976 IEEE MTT-S Microwave Symp., 14-16 June, 1976,

Cherry Hill, NJ, pp. 224-226 1976

IEEE Trans. Instrum. Meas., Vol. IM-25, No. 3, pp. 173-182 September 1976

RANDA, M.; LARSON, T. R.

Stability of Silicon Avalanche Noise Diodes at $\mathrm{K}$ and $\mathrm{Ka}$ Bands Proc., 1976 Conf. on Precision Electromagn. Meas.,

28 June-1 July, 1976, Boulder, CO 1976

MILLER, C. R. S.; DAYWITT, W. C.

The NBS Switching Radiometers

NBSIR 84-3004

May 1984

The Reference Noise Standards NBS WR62 and WR90

NBSIR 84-3005

May 1984

MILLER, C. K. S.; DAYWITT, W. C.; ARTHUR, M. G.

Noise Standards, Measurements, and Receiver Noise Definitions

Proc., IEEE, Special Issue on Radio Meas. Methods and Standards, Vol. 55, No. 6, pp. 865-877

June 1967

PERERA, $\mathbf{S}$.

Noise Temperature Measurements at the National Bureau of Standards

Proc., 1985 Instrum. and Meas. Technol. Conf.,

20-22 March, 1985, Tampa, FL, pp. 159-160 1985

A Simple Noise Calibration Radiometer

Digest, CPEM; 11-14 June 1990; Ottawa, Canada; pp. 32-33 June 1990

WAIT, D. F.

Considerations for the Precise Measurement of Amplifier Noise NBS Tech. Note 640 1973 
WAIT, D. F. (cont.)

Earth Terminal Measurement system Operations Manual

NBSIR 78-879

April 1978

Precision Measurement of Antenna System Noise Using Radio Stars Dig., 1982 Conf. Prec. Electromagn. Meas.,

28 June-1 July, 1982, Boulder, CO, p. F-17 1982

IEEE Trans. Instrum. Meas., Vol. IM-32, No. 1, pp. 110-116 March 1983

Earth Terminal Measurement system Operations Manual (Revised)

NBSIR 83-1679(R) January 1983

The Impact of Automation on NBS Noise Temperature Measurements

Proc., IEEE Spec. Issue Radio Meas. Methods Standards, pp. 117-120 1986

Comments Concerning On-Wafer Noise Parameter Measurements

Digest, 36th ARFTG Conf., 29-30 Nov. 1990, Monterey, CA, pp. 5-15 November 1990

WAIT, D. F.; DAYWITT, W. C.

Preliminary Examination of $20 \mathrm{GHz}$ G/T Measurements of Earth Terminals NBSIR 83-1686 March 1983

Intercomparison of NBS Noise Calibration Services

Dig., Conf. Prec. Electromagn. Meas., 7-10 June, 1988, Tsukuba Science City, Japan, pp. 209-210 1988

WAIT, D. F.; ENGEN, G. F.

Application of Radiometry to the Accurate Measurement of Amplifier Noise IEEE Trans. Instrum. Meas., Vol. IM-40, No. 2, pp. 433-437 April 1991

WAIT, D. F.; COUNAS, G. J.; RESSEL, W.; BUCHHOLZ, F. IM.

PTB - NIST Bilateral Comparison of Microwave Noise Power in Coaxial Transmission Line.

Dig., Conf. Prec. Electromagn. Meas., 11-14 June 1990; Ottawa, Canada, pp. 34-35. June 1990

IEEE Trans. Instrum. Meas., Vol. IM-40, No. 2, pp. 449-454 April 1991

WAIT, D. F.; RANDA, M.; DAYWITT, W. C.; MILLER, C. K. S.

A study of the Measurement of G/T Using Cassiopeia A

NBSIR 74-382

october 1974

WAKEFIELD, J.P.

Addendum to Earth Terminal Measurement system Maintenance Manual NBSIR 81-1641

October 1981 
BELSHER, D. R.

Feasibility Study on the use of a Microwave System for the

Nondestructive Evaluation of Historic Adobe structures

NBSIR 79-1610

July 1979

BELSHER, D. R.; MCLAUGHIN, R. G.; REPJAR, A. G.; BUSSEY, H. E.

Detection of Lost Wells and Unknown Water-filled Voids in Coal Mines

through Development of an Experimental Microwave System NBSIR 84-3017

September 1984

BEREUTER, W. A.: CEANG, D. C.

Electromagnetic Remote Sensing of Inhomogeneous Media NBSIR 76-851

January 1977

BOYNE, H. S.: ELLERBRUCH, D. A.

Microwave Measurements of Snow Stratigraphy and Water Equivalence Proc., The Western snow Conference

April 1979

Chang, A. T. C.; SHIUE, J. C.; BOYNE, H. S.; ELLERBRUCE, D. A.;

COUNAS, G. J,

Preliminary Results of Passive Microwave Snow Experiment During February and March 1978

NASA-TP-1408, Rept $-7802-F 22$, p. 114 1979

CEEW, $\mathbf{H}$.

Modeling of Oil shale Retorts for Electromagnetic Sensing Techniques NBSIR 81-1653 November 1981

Electromagnetic Modeling of Oil shale Retorts for Remote Sensing Purposes

IEEE Trans. Geosci. Remote Sensing, Vol. GE-20, No. 4, pp. 510-517

october 1982

COLLIER, R. S.; ELLERBRUCH, D. A.

RF (Radiofrequency) Total Mass Gauging in Large storage Containers:

Empty Tank Modes

NBSIR 73-346

October 1973

COLIIER, R. S.; ELLERBRUCE, D. A.; CRUZ, J. E.; STORES, R. W.;

LUFT, $\mathbf{P}$. E.

Mass Quantity Gauging by RF Mode Analysis

NBSIR 73-318

June 1973

CRUZ, J. E.; ROGERS, E. H.; HIESTER, A. E.

Continuous Liquid Level Measurements with Time-Domain Reflectometry

Adv. Cryo. Eng., Vol. 18, pp. 323-327 (Paper H-4) 1973

BLIERBRUCH, D. A.

Electromagnetic Attenuation Properties of Clay and Gravel Soils NBSIR 74-381

August 1974 
ELLERBRUCH, D. A.; ADAMS, J . W.

Microwave Measurement of Coal Layer Thickness

NBSIR 74-387

September 1974

ELLERBRUCH, D. A.; BELSHER, D. R.

FM-CW Electromagnetic Technique of Measuring Coal Layer Thickness NBSIR 76-840

May 1976

Electromagnetic Technique of Measuring Coal Layer Thickness

IEEE Trans. Geosci. Electron., GE16, No. 2, pp. 126-133 April 1978

ELLERBRUCH, D. A.; JESCH, R. L.; JONES, R. N.; BUSSEY, H. E.; BOYNE, H. S.

Electromagnetic scattering Properties of Soils and snow Proc., 1978 Intl. Symp. on Remote Sensing of Environment (12th), 20-26 April, 1978, Ann Arbor, MI, Vol. 2, pp. 957-974 1978

ELIERBRUCH, D. A.; JOHNSON, R. B.; BELSHER, D. R.; YAGHJIAN, A. D.; STEPPE, M. C.; FLEMING R. W.

High Resolution Sensing Techniques for slope stability studies Federal Highway Administration Report, FHWA-7-3-0001 March 1979

GANS, W. I.; GEYER, R. G.; REMPERER, W. R.

Quantifying Standard Performance of Electromagnetic-Based Mine Detectors NISTIR 3982

October 1991

HIIL, D. A.

Radio Propagation in a coal seam and the Inverse Problem NBS JRES, Vol. 89, No. 5, pp. 385-394

September-October 1984

Proc., 1985 Intl. Symp. on Antennas and EM Theory,

26-28 August, 1985, Beijing, China, pp. 422-427 1985

Electromagnetic Wave Propagation in a Symmetrical coal Seam

IEEE Trans. Antennas Propagat., Vol. AP-34, No. 2, pp. 244-247 February 1986

Fields of Horizontal Currents Located Above the Earth

IEEE Trans. Geosci. Remote Sensing, Vol. 26, No. 6, pp. 726-732

November 1988

Magnetic Dipole Excitation of a Long Conductor in a Lossy Medium IEEE Trans. on Geoscience and Remote Sensing, Vol. 26, No. 6, pp. $720-725$

November 1988

Magnetic Dipole Excitation of an Insulated Conductor of Finite Iength

IEEE Trans Geosci. Remote Sensing, Vol. 28, No. 3, pp. 289-294 May 1990 
HILL, D. A.; ANDERSON, L. R.

Propagation Along a Two-Wire Line Located at the Air-Earth Interface IEEE Trans. on Geosci. Remote Sensing, Vol. 28, No. 3 pp. 400-402.

May 1990

MONTGOMERY, J. P.; CHANG, D. C.

Electromagnetic Boundary-Value Problems Based Upon a Modification of Residue Calculus and Function Theoretic Techniques NBS MN 164

June 1979

ROE, R. C.; WITMTANA, R. C.

Improved Coal Interface Detector NBSIR 82-1663

May 1982

STOLTENBERG, R. E.

Detection of Human Intruders by Low Frequency Sonic Interferometric Techniques

NBSIR 74-364

May 1974 
ADAMS, J.W.; ONDREJKA, A. R.; MEDLEY, B. W. Time-Domain System for Indentification of the Natural Resonant Frequencies of Aircraft Relevant to Electromagnetic Compatibility Testing NBSIR $87-3077$

November 1987

ADAMS, J. W.; ONDREJRA, A. R.; CAVCEY, K. H.; CRUZ, J. E.; MEDLEY, H. W.; GROSVENOR, J B.

Recent Improvements in Time-Domain EMC Measurement system NISTIR 89-3927 November 1989

ANDREWS, $\mathbf{J}, \mathbf{R}$.

Impulse Generator Spectrum Amplitude Measurement Techniques IEEE Trans. Instrum. Meas., Vol. IM-25, No. 4, pp. 380-384 December 1976

ANDREWS, J.R.; BAIDWIN, E. E.

Baseband Impulse Generator Useful to $5 \mathrm{GHz}$ Proc., 1975 IEEE Intl. Symp. on Electromagn. Compat., 7-9 October, 1975, San Antonio, TX, pp. 1-4 1975

ANDREWS, J. R.; GANS, W. L.

Time Domain Automatic Network Analyzer

L'Onde Electrique, Vol. 55(10), pp. 569-574 December 1975

GANS, W. L.; ANDREWS, J.R.

Time Domain Automatic Network Analyzer for Measurement of RF and Microwave Components

NBS Tech. Note 672

september 1975

GANS, W. L.; GEYER, R. G.; RLEMERER, W. R.

Suggested Methods and Standards for Testing and Verification of Electromagnetic Buried Object Detectors NISTIR 88-3915 March 1990

GEYER, R. G. Magnetostatic Measurements for Mine Detection;

NISTIR 88-3098 October 1988

RANDA, M.; ONDREJRA, A. R.

An Ultra-Broadband and Nondispersive Sensor for the Measurement of TimeDomain Signals

Proc., symp. on Electromagnetic security for Information

Protection, 21-22 Nov., 1991, Rome, Italy, pp. 65-78 November 1991

LAWTON, R. A.; ONDREJRA, A. R.

Standards for the Measurement of Impulsive Fields Radiated by a TEM Horn Antenna

Proc., 1977 Union Radio Scientifique Intl. E Comm.,

3-7 October, 1977, Lannion, France, pp. 1-4

1977 
LAWTON, R. A.; RIAD, S. M.; ANDREWS, J.R.

Pulse and Time Domain Measurements Proc., IEEE, Special Issue on Radio Meas. Methods and Stds, Vol. 74, No. 1, pp. 77-81 January 1986

NABMAN, N. S.: JICKLING, R. M.

Frequency Domain Measurement of Baseband Instrumentation NBSIR 73-330

July 1973

NARMAN, N. S.; JICRLING, R. M.; HOLT, D. R.

Reference-Waveform Generation Using Debye Dielectric Dispersion NBSIR 73-304

December 1972

TOFANI, S.; ONDREJRA, A. R.; RANDA, M.

A Time-Domain Method for Characterizing the Reflection Coefficient of Absorbing Materials from 30 to $1000 \mathrm{MHz}$

IEEE Trans. Electromagn. Compat., Vol. 33, No. 3, pp. 234-240 August 1991 
ANDREWS, J.R.

Improved Bias Supply for Tunnel-Diode Picosecond Pulse Generators IEEE Trans. Instrum. Meas., IM-19, pp. 171-175

August 1970

Horizontal Time Base Sweep Generator for a Traveling Wave Oscilloscope IEEE Trans. Nucl. Sci., NS-18, pp. 3-18 October 1971

An Interfacing Unit for a 28 psec Feedthrough Sampling Oscilloscope Rev. Sci. Instrum., Vol. 42 , pp. 1882-1885 December 1971

A Frequency Calibrator for UHF Using an Avalanche Transistor Q S T, Vol. 56, pp. 16-18 May 1972

Random Sampling Oscilloscope Time Base NBSIR 73-309 June 1973

Random Sampling Oscilloscope for the Observation of Mercurÿ Switch Closure Transition Times IEEE Trans. Instrum. Meas., IM-22, pp. 375-381 December 1973

Inexpensive Laser Diode Pulse Generator for Optical Waveguide Studies Rev. Sci. Instrum., Vol. 45, pp. 22-24 January 1974

Precision Picosecond-Pulse Measurements Using a High Quality Superconducting Delay Line

Proc. Conf. Prec. Electromagn. Meas., 1-5 July, 1974, London, England, pp. 316-318 1974

IEEE Trans. Instrum. Meas., IM-23, pp. 468-472 December 1974

Directional-Coupler Technique for Triggering a Tunnel Diode IEEE Trans. Instrum. Meas., IM-24, pp. 275-277 September 1975

Impulse Generator spectrum Amplitude Measurement Techniques

Dig., Conf. Prec. Electromagn. Meas., pp. 23-24 1976

Measurement on Pulses and Pulse Transmission Media, Circuits and Components

Intl. Union of Radio sci. (URSI) Review of Radio science, p. 9 1975-1977

The Estimation of the Pulse Waveform in the Calibration of Impulse Generators

Dig., Intl. Union of Radio sci. (URSI) Meeting, January, 1981, Boulder, CO, p. 87 1981

Pulse Reference Waveform Standards Development at NBS

Proc., ATE Seminar/Exhib., January, 1981, Pasadena, CA, p. IV.13 through IV. 19

1981 
ANDREWS, J. R.; BALDWIN, E. E.

Amplitude Calibrator for Oscilloscopes NBSIR 81-1646

April 1981

ANDREWS, J. R.; GANS, W. I.

Pulsed Wavemeter Timing Reference for Sampling Oscilloscope Calibration IEEE Trans. Instrum. Meas., IM-24, p. 82 March 1975

ANDREWS, J.R.; IAWTON, R. A.

Picosecond Pulse Research at NBS

Proc., Joint Meas. Conf., 12-14 November, 1974, Gaithersburg, MD, pp. $123-140$ 1974

Electrically strobed optical Waveform Sampling Oscilloscope

Rev. Sci. Instrum., Vol. 47, pp. 311-313 March 1976

ANDREWS, J. R.; NAHMAN, N. $\mathbf{s}$.

Electron-Beam Deflection in Traveling-Wave Oscilloscopes

Record of Symp. on Electron, Ion, and Laser Beam Technol. (11th), 12-14 May, 1971, Boulder, CO

1971

The Measurement of Pulse Transition Duration

Proc., Union Radio Scientifique Internationale Comm. Symp.,

3-7 october, 1977, Lannion, France, pp. 159-164 1977

ANDREWS, J.R.; BELL, B. A.; BALDWIN, E. E.

Reference Flat Pulse Generator

NBS Tech. Note 1067

October 1983

ANDREWS, J.R.; NAHMAN, N. S.; BELL, B. A.

Status of Reference Waveform Standards Development at NBS

NBS SP 634, Proc., 1981 Seminar on Waveform Recorder Measurement Needs and Techn. for Evaluation/Calibration,

15 October, 1981, Boulder, CO, pp. 69-88

1982

ANDREWS, J.R.; WHITTEMORE, T.; MCCAA, W. D. Jr.

Survey of Present Waveform Sampling system Limitations

NBS Rept. 10731

February 1972

ANDREWS, J. R.; BELL, B. A.; NAHMAN, N. S.; BALDWIN, E. E.

Reference Waveform Flat Pulse Generator

Dig., Conf. Prec. Electromagn. Meas. Conf., June, 1982 1982

IEEE Trans. Instrum. Meas., Vol. IM-32, No. 1, pp. 27-32 March 1983

BOUILLE, R.; ANDREWS, J.R.

Measurement of Broadening of Pulses in Glass Fibers

Electron. Lett., Vol. 8, pp. 309-310

June 1972 
CHESNUT, S. M.; PAULTER, N. G., Jr.

Automatic Waveform Analysis and Measurement System User Manual NISTIR 3978

December 1991

ERSTROM, M. P.; MCCAA, W. D. JY.; NAHMAN, N. S.

The Measured Time and Frequency Response of a Miniature Superconducting Coaxial Line

IEEE Trans. Nucl. Sci., NS-18, pp. 18-25 October 1971

GANS, W. L.

Present Capabilities of the NBS Automatic Pulse Measurement System

Dig., Conf. Prec. Electromagn. Meas., pp. 25-26 1976

IEEE Trans. Instrum. Meas., IM-25, pp. 384-388 December 1976

The Measurement and Deconvolution of Time Jitter in Equivalent-Time Waveform Samplers

IEEE Trans. Instrum. Meas., Vol. IM-32, No. 1, pp. 126-133

March 1983

Picosecond Pulse Measurements at NBS

Proc., 1985 Instrum.-and Meas. Technol. Conf., 20-22 March, 1985, Tampa, FL, pp. 142-144 1985

Picosecond Pulse Waveform Error Analysis at NBS

Proc., IEEE Spec. Issue on Radio Meas. Methods and Standards, pp. $86-90$ January 1986

Dynamic Calibration of Oscilloscopes and Waveform Recorders Using Pulse standards.

Record, Instrumentation \& Measurement Technology (IMTC) Conf.; 1315 February 1990; San Jose, CA; pp. 246-250. February 1990

GANS, W. L. ; NARMAN, N. S.

Pulse Testing of $R F$ and Microwave Components

NBSIR 1973

July 1973

Shielded Balanced and Coaxial Transmission Lines - Parametric Measurements and Instrumentation Relevant to Signal Waveform Transmission in Digital Service

NBSIR 81-1042

June 1981

HOLT, D. R.; NARMAN, N. $S$.

Coaxial Line Pulse-Response Error Due to a Planar Skin Effect

Approximation

Proc., 1972 Conf. Prec. Electromagn. Meas., 26-29 June, 1972,

Boulder, $\mathrm{CO}$, pp. 1-2 1972

IEEE Trans. Instrum. Meas., IM-21, pp. 515-519

November 1972 
BONDA, T.; NABMAN, N. S .

A Dye Modelocked Nd+3 Glass Laser for Generating Electrical Reference Waveforms

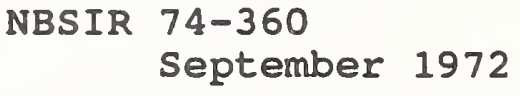

Application of Ultrashort Optical Pulses to Electrical Pulse Measurements

Proc. Symp. on New Instrum., 28-29 January, 1974, IEE of Japan, Hokkaido University, pp. 29-38 1974

BUDSON, P. A.; ANDREWS, J. R.; BLANR, W.; ONDREJRA, A. R.

Investigation and Evaluation of Methods for Pulse Rise-Time Measurement Tech. Report RADC-TR-68-207 October 1968

KANDA, M.

Time Domain Sensors and Radiators

Natl. Radio Science Meeting, January, 1982, University of

Colorado, Boulder, CO 1982

LAWTON, R. A.

Measurement Needs and Techniques for Evaluation/Calibration, Proc., 1981 Seminar on Waveform Recorder, 15 october 1981 , Boulder, Co NBS SP 634

1982

Precision Picosecond-Microsecond Electromagnetic Waveform Measurement at NBS

NBS SP 628, pp. 392-407 June 1982

Performance Standards for Waveform Recorders

IEEE Trans. Nucl. Sci., Vol. NS-30, No. 1, pp. 263-266 February 1983

Photoconductive Switches Used for Waveform Generation at the National Bureau of Standards

Proc., 1983 Picosecond Optoelectronics Conf., SPIE - The

International Society for Optical Engineering, 24-26 August, 1983,

San Diego, CA, Vol. 439, pp. 88-94 1983

An Efficient Antialiasing Filter

Dig., 1984 Conf. on Precision Electromagn. Meas., 20-24 August, 1984, Delft, The Netherlands, pp. 104-105 1984

IEEE Trans. on Instrum. and Meas., Vol. IM-34, No. 4, pp. 570-573

December 1985

Pulse Waveform Standards for Electro-Optics

Proc., 1985 Topical Meeting on Picosecond Electronics and Opto-Electronics, 13-15 March, 1985, Lake Tahoe, NV, pp. 205-206

1985 
LAWTON, R. A. (cont.)

Status Report Transient Waveform Recorder Standard

Proc., 1985 Instrum. and Meas. Technol. Conf., 20-22 March, 1985, Tampa, FL, pp. 154-155 1985

LAWTON, R. A.; ANDREWS, J. R. Pulsed-Laser Application to Sampling Oscilloscope Electron. Lett., vol. 11, pp. 138 April 1975

Electrical Strobing of Photoconductor Cuts Sampling Oscilloscope's Risetime

Laser Focus, pp. 62-65

November 1976

LAWTON, R. A.; MEYER, R.

Waveform Standards for Electro-Optics: A Pulse Duration Comparison IEEE Trans. on Microwave Theory and Techn., Vol. MTT-35, No. 4, pp. 450-453

April 1987

LAWTON, R. A.; NABMAN, N. S.

Pyroelectric Detector Application to Baseband Pulse Measurements Electron. Lett., Vol. 8, pp. 318-330 June 1972

LAWTON, R. A.; NABMAN, N. S.; BIGELOW, J. M.

A Solid State Reference Waveform Standard

Dig., Instrum. and Meas. Technol. Conf., 17-18 January, 1984, Long

Beach, CA, pp. 74-76 1984

IEEE Trans. Instrum. Meas., Vol. IM-33, No. 3, pp. 201-205 September 1984

Reference Waveform Standard

Dig., IEEE Trans. Instrum. Meas., Vol. IM-33, No. 3,

pp. 201-205

September 1984

LAWTON, R. A.; SCAVENNEC, A.

Photoconductive Detector of Fast-Transition Optical Waveforms

Electron. Lett., Vol. 11, pp. 74-75

February 1975

LAWTON, R. A.; YOUNG, M.

Laser-Mode Beating Used for Detector Frequency-Response Measurements

Appl. Opt., Vol. 16, pp. 2703-2705

October 1977

MCCAA, W. D. Jr.; NABMAN, N. S .

Generation of Reference Waveforms by Uniform Lossy Transmission Lines

IEEE Trans. Instrum. Meas., IM-19, pp. 382-390 November 1970

NAGMAN, N. S.

The Measurement of Baseband Pulse Rise Times of Less than $10^{-9}$ Second Proc., IEEE, Special Issue on Radio Meas. Methods and Standards, Vol. 55, No. 6, pp. 855-864 June 1967 
NABMAN, N. S. (cont.)

Picosecond Domain Waveform Measurements (Japanese translation)

Nikkei Electron., Nikkei-McGraw Hill

April 1979

Error Criteria and the Use of Reference Waveforms

Proc. ATE Seminar/Exhib., January, 1981, Pasadena, CA,

pp. IV.21 through IV.28

1981

Error Criteria and the Use of Reference Waveforms

Electron. Test, Vol. 4, No. 2, pp. 72-76

February 1981

Some Generic Waveform Recorder Problems

NBS SP 634, Proc., 1981 Seminar on Waveform Recorder Measurement Needs and Techn. for Evaluation/Calibration, 15 October, 1981, Boulder, CO, pp. 1-5 1982

Picosecond Domain Waveform Measurements; Status and Future Directions

IEEE Trans. Instrum. Meas., Vol. IM-32, No. 1, pp. 117-124 March 1983

Software Correction of Measured Pulse Data

NATO ASI Series on Fast Electrical and Optical Meas., Vol 1,

Series E, No. 108, pp. 351-417 1986

Picosecond-Domain Waveform Measurements

Proc., IEEE, Vol. 66, pp. 414-423

April 1978

NABMAN, N. S.; GUILLAUME, M. E.

Deconvolution of Duration Limited Waveforms

Dig. of Papers, URSI/U. S. National Radio Sci. Meeting,

January 1981, Boulder, CO, p. 86 1981

NAHMAN, N. S.; HOLT, D. R.

Transient Analysis of Coaxial Cables Using the Skin Effect Approximation

$A+B$ (the square root of $s$ )

IEEE Trans. Circuit Theory, CT-19, pp. 443-451

September 1972

NABMAN, N. S . ; ERSTROM, M. P.; MCCAA, W. D. Jr.

Measured Time and Frequency Response of a Miniature Superconducting

Coaxial Line

Applied Superconductivity Conf., June 1970, Boulder, CO 1970

ONDREJRA, A. R.; RANDA, M.

A Time-Domain Method for Measuring the Reflection Coefficient of Microwave Absorbers at Frequencies Below $1 \mathrm{GHz}$

Digest, 1991 Antennas and Propagation Society Symp., 24-28 June, 1991, London, Ontario, Canada, pp. 1656-1663, Vol. 3 1991 
PAULTER, N. G., Jr.; MAJUMDAR, A.

A New Proposed Triple Correlator for the Measurement of Ultrashort Laser Pulses

Opt. Comm., Vol. 81, No. 1,2, pp. 95-100

February 1991

A New Triple Correlation Technique for Measuring Ultrashort Laser Pulses Rev. Sci. Instrum., Vol. 62, No. 3, pp. 567-578 March 1991

RIAD, S. M.

The Deconvolution Problem: An Overview

Proc. of the IEEE, Special Issue on Radio Meas. Methods and

Standards, pp. 82-85 January 1986

RIAD, S. M.; NARMAN, N. S .

Application of the Homomorphic Deconvolution for the separation of TDR Signals Occurring in Overlapping Time Windows

CPEM Dig., pp. 27-29 1976

IEEE Trans. Instrum. Meas., IM-25, pp. 388-391 December 1976

SCAVENNEC, A.; NAEMAN, N. S .

Active and Passive Mode Locking of Continuously Operating Rhodamine 6G Dye Lasers NBSIR 73-347

February 1974

TOFANI, S.; ONDREJKA, A. R.; KANDA, M.

A Time-Domain Method for Characterizing the Reflection Coefficient of Absorbing Materials from 30 to $1000 \mathrm{MHz}$

IEEE Trans. on Electromagnetic Compatibility, Vol. 33, No. 3, pp. 234-240

August 1991

YOUNG, M.; LAWTON, R. A.

Measurement of Pulsed-Laser Power

NBS Tech. Note 1010

February 1979 


\section{MISCELILANEOUS}

ARTEUR, M. G.; REEVE, G. R. Modulation Factor standards NBS Tech. Note 1016

september 1979

BAIRD, R. C.

RF Measurements of the speed of Light

Proc., IEEE, Special Issue on Radio Meas. Methods and Standards, Vol. 55, No. 6, pp. 1032-1039 June 1967

BENSEMA, W. D.

Personal FM Transceivers

National Institute of Justice standard-0209.01 september 1985

Mobile FM Transceivers National Institute of Justice Technology Program, NIJ Report -210.00 May 1986

BERRY, L. A.; FITZGERRELI, R. G.; VOGLER, I. E.

Investigation of Effect of Antenna site and Antenna Type on LF Non-Directional Beacon Performance FAA-RD-73-174 1973

BOWMAN, $R$. $R$. Self-Modulating Transducers EOS-7039-Final 1968

A Temperature Probe for Radio-Frequency Heated Material NBSIR $81-1634$ January 1981

BRANDENBERGER, H.; HADORN, F.; HALFORD, D.; SHOAF, J. B. High Quality Quartz Crystal Oscillators: Frequency Domain and Time Domain stability Proc., 1971 Frequency Control Annual symp. (25th), 26-28 April, 1971, Atlantic City, NJ, pp. 226-234 1971

BUSSEY, B. E.; LARSEN, E. B. Buried Antenna Performance: Development of Small Resonant Buried Antennas RADC-TR-74-169M, AD 783274 June 1974

CBEW, H.

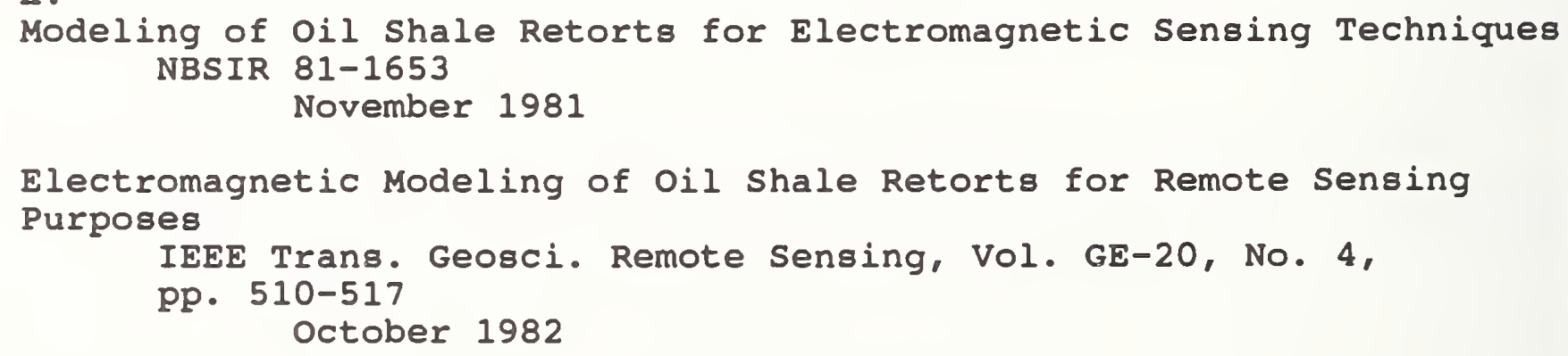


CLARR, B. E.

Requirements for an Effective National Nonionizing Radiation Measurement system

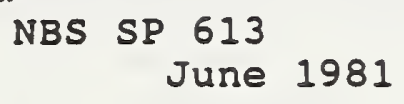

COLIIER, R. S.; CRUZ, J. E.; ELLERBRUCH, D. A.; LOWE, L. T.; WEITZEL, D. H. Instrumentation for Hydrogen slush storage Containers

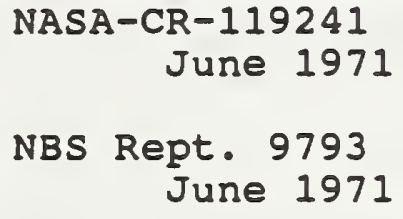

DOMICE, P. D.; BAKER-JARVIS, J. R.; GEYER, R. G.

Optimization Techniques for Permittivity and Permeability Determination NIST JRES, Vol. 96 , No. 5, pp. 565-575

september-october 1991

DRIVER, L. D.; RIES, F. $\mathbf{I}$.

A Wideband RF Voltage Comparator

Dig., 1980 Conf. Precision Electromagn. Meas. June 1980

ELLERBRUCB, D. A.

Microwave Methods for Cryogenic Liquid and Slush Instrumentation

IEEE Trans. Instrum. Meas., Vol. IM-19, No. 4, pp. 412-416 November 1970

Application of Measurements of Electromagnetic Phenomena to Oceanography Proc., 1972 UJNR/MEC Symp. (1st), Record of the U.S.-Japan UJNR Joint symp. on Marine Electronics, (Japanese Electrical Industrial Committee, Tokyo, Japan), 9 October, 1972, Tokyo, Japan, pp. 23-30 1972

Test Results for the Mooring Iine Data Iine NBSIR 73-341

October 1973

ELLERBRUCH, D. A.; BOYNE, H. S.

Active Microwave Water Equivalence Measurements

Proc., Ft. Collins snow Workshop 1980

FITZGERREIL, R. G.

Mobile Antennas

National Institute of Justice standard-0205.01 May 1989

FOOTE, W. J.

A New Flange Design for o-Ring Seals

Rev. Sci. Instrum.

August 1970

GIBSON, R. A.; MILIER, C. R. S.

Bibliography of the Electromagnetic Fields Division Publications NBSIR 82-1673

August 1982

A Bibliography of the NBS Electromagnetic Fields Division Publications NBSIR 85-3022

April 1985 
GIBSON, R. A.; PAGE, J. M.; MILIER, C. K. S.

A Bibliography of the NBS Electromagnetic Fields Division Publications NBSIR 85-3040

February 1986

BALFORD, D.

Infrared-Microwave Frequency Synthesis Design: Some Relevant Conceptual Noise Aspects

Proc., 1971 Frequency Standards and Metrology Seminar,

30 August-1 September, 1971, Quebec, Canada, pp. 431-466 1972

HALFORD, D.; SBOAF, J. B.; RISLEY, A. S.

Spectral Density Analysis: Frequency Domain specification and Measurement of Signal stability

1973 Annual Symp. on Frequency Control (27th), 12-14 June, 1973, Cherry Hill, NJ, pp. 421-431 1973

HAMILTON, C.; ILOYD, F.; PETERSON, R.; ANDREWS, J . R.

A Superconducting Sampler for Josephson Logic Circuits

Appl. Phys. Lett., Vol. 35, No. 9, pp. 718-719

November 1979

HAYES, R. E.; RANDA, M.; MASCLET, G.; MAY,. .

The Application of Semiconductors to Quasi-optical Isolators for use at Submilimeter Wavelengths

$$
\text { NASA-CR-105980 }
$$

July 1969

NASA-CR-86379

April 1970

HIIL, D. A.

HF Ground Wave Propagation over Forested and Built-up Terrain NTIA Report 82-114

December 1982

Book Review - Leaky Feeders and Subsurface Radio Communications IEEE Antennas Propagat. Soc. News., Vol. 25, No. 3, pp. $23-24$

June 1983

HIL, D. A.; WAIT, J.R.

Propagation Along a Coaxial Cable with a Helical Shield

IEEE Trans. Microwave Theory Technol., Vol. MTT-28, No. 2, pp. 84-89

February 1980

Electromagnetic Theory of the Loosely Braided Coaxial Cable: Part II Numerical Results

IEEE Trans. Microwave Theory Technol., Vol. MTT-28, No. 4,

pp. 326-331

April 1980

Ground Wave Attenuation Function for a spherical Earth with Arbitrary Surface Impedance Radio Science

Radio Science, Vol. 15, No. 3, pp. 637-643

May-June 1980

On the Excitation of the Zenneck Surface Wave Over the Ground at $10 \mathrm{MHz}$ Annales des Telecommunications, Vol. 35, Nos. 5-6, pp. 1-4 May-June 1980 
HILI, D. A.; WAIT, J.R. (cont.)

Electromagnetic Characteristics of a Coaxial Cable with Periodic slots IEEE Trans. Electromagn. Compat., Vol. EMC-22, No. 4, pp. 303-307 November 1980

HF Ground Wave Propagation over sea Ice for a spherical Earth Model IEEE Trans. Antennas Propagat., Vol. AP-19, No. 3, pp. 525-527 May 1981

HF Ground Wave Propagation over Mixed Land, Sea, and Sea-Ice Paths IEEE Trans. Geosci. Remote Sensing, Vol. GE-19, No. 4, pp. $210-216$

october 1981

HF Radio Wave Transmission over Sea-Ice and Remote sensing Possibilities IEEE Trans. Geosci. Remote Sensing, Vol. GE-19, No. 4, pp. 204-209

October 1981

Ground Wave Propagation over a Mixed Path with an Elevation Change IEEE Trans. Antennas Propagat., Vol. AP-30, No. 1, pp. 139-141

January 1982

JESCE, R. I.

Evaluation of Low-Loss/Low-Reflection Two-Port Devices or Adapters by Automated Measurement Techniques NBSIR 78-870

December 1977

Dielectric Measurements of Oil shale as Functions of Temperature and Frequency NBSIR 83-1683 January 1983

Measured Vehicular Antenna Performance

IEEE Trans. Veh. Technol., Vol. VT-34, No. 2, pp. 97-107 May 1985

Personal/Mobile FM Transceivers

National Institute of Justice Technology Program, NIJ Report-0224.00 May 1986

Fixed and Base station FM Transmitters National Institute of Justice standard - 0201.01 september 1987

Fixed and Base station FM Receivers National Institute of Justice Standard-0206.01 July 1988

JESCH, R. L.; BERRY, I. S.

Batteries used with Law Enforcement Communications Equipment NBS Rept. 10722 October 1971

JESCH, R. L.; HOER, C. A. Characterization of a High Frequency Probe Assembly for Integrated Circuit Measurements NBS Tech. Note 663 January 1975 
JESCH, R. I.; BAILEY, R. A.; TAUSCH, H. J.

Characterization of A H.F. Probe for Integrated Circuits Measurements Proc., Government Micro-Circuit Applications Conf.,

25-27 June, 1974, Boulder, CO, pp. 120-121 1974

JICRIING, R. M.; SEAFER, J.F.

Repeaters for Law Enforcement Communication Systems-Law Enforcement Standard Program

Law Enforcement Standards Laboratory RPT-0206.00 october 1974

FM Repeater systems

National Institute of Law Enforcement and Criminal Justice, STD-0213.00 November 1977

JONES, R. N.

A Comparison of Centrifuge and Freezing Calorimeter Methods for

Measuring Free Water in snow

NBSIR 79-1604

April 1979

Evaluation of Three-Terminal and Four-Terminal Pair Capacitors at High Frequencies

NBS Tech. Note 1024

September 1980

RANDA, $\mathbf{M}$.

Non-Reciprocal Devices Using Solid State Magnetoplasmas at Millimeter and Submillimeter Wavelengths

NASA-CR-122923

August 1971

RANDA, M.; MAY, W. G.

Hollow-Cylinder Waveguide Isolators for use at Millimeter Wavelengths NASA-CR-140760 November 1974

Reflection Beam Isolator for Submillimeter Wavelength NASA-CR-140761 June 1975

RERNS, D. M.

Scattering-Matrix Description and Near-Field Measurements of Electroacoustic Transducers

J. Acoust. Soc. Amer., Vol. 57, No. 2, pp. 497-507 February 1975

KING, J.; PEISER, H. S.; SANGSTER, R. C.

Guidelines for specification and Procurement of Measurement

Instrumentation

NBSIR 78-891

June 1978

KUFFEL, J.; MALEWSKI, R.; VAN GEESWIJK, R.; IAWTON, R. A. Dynamic Performance of Digital Recorders Used for Monitoring High Voltage Impulse Tests

Proc., 1985 Instrum. and Meas. Technol. Conf.,

20-22 March, 1985, Tampa, FL, pp. 211-215 1985 
LARSEN, N. T.; VECCEIA, D. F.; SUGAR, G. R. VOR Calibration Services

NBS Tech. Note 1069

April 1985

LAWTON, R. A.; ANDREWS, J.R.

Optically strobed Sampling Oscilloscope

IEEE Trans. Instrum. Meas., 25, pp. 56-60

March 1976

LEWIS, R. L。

The Wave-Hop Fields for an Inclined Dipole Over a Spherical Earth with

an Anisotropic Ionosphere

OT-ITR-RR 5

October 1970

Spherical Scanning Data Processing: An Algorithm for Halving the Data Processing Effort When the Radiation into the Back Hemisphere is Negligible

Dig., 1981 IEEE Ant. Propagat. Soc. Intl. Symp., June 1981 1981

LITILE, W. E.

Automated Computer Controlled Measurements

Proc., 1974 Conf. Preci. Electromagn. Meas., 1-5 July, 1974,

London, England 1974

LYONS, R. M.; GIBSON, R. A.

A Bibliography of the NIST Electromagnetic Fields Division Publications NISTIR 89-3920

September 1989

A Bibliography of the NIST Electromagnetic Fields Division Publications NISTIR 3945

August 1990

A Bibliography of the NIST Electromagnetic Fields Division Publications NISTIR 3973

August 1991

MANNEY, C. B.

Applicability of Speech Bandwidth Compression Techniques in Mine

Electromagnetic Communications

NBS Rept. 10728

February 1972

MILLER, C. R. S.

Millimeter Wave Metrology Capabilities at NBS

Proc., 1974 Millimeter Waves Techniques Conf.,

26-28 March, 1974, San Diego, CA, p. 11 1974

A Bibliography of Publications in the NBS Electromagnetic Fields Division

NBSIR 80-1635

November 1980

MONTGOMERY, J. P.; CHANG, D. C.

Electromagnetic Boundary-Value Problems Based Upon a Modification of

Residue Calculus and Function Theoretic Techniques NBS MN 164

June 1979 
NABMAN, N. S.; AILRED, C. M.; ANDREWS, J. R.; HOER, C. A.; IAWTON, R. A. Radar Absorber Measurement Techniques at Frequencies Above $20 \mathrm{GHz}$ NBSIR 79-1613

August 1979

NELSON, R. E.

A Guide to Voice Scramblers for Law Enforcement Agencies NBS SP 480-8

December 1976

NELSON, R. E.: JICKING, R. M.

Control Cable Assemblies Used with Mobile FM Receivers National Institute of Justice, NIJ Std-0216.00 December 1981

REIDY, A. R.; GIBSON, R. A.

A Bibliography of NIST Electromagnetic Fields Division Publications NISTIR 88-3900 September 1988

ROE, R. C.; WITMMANN, R. C.

Improved Coal Interface Detector NBSIR 82-1663

May 1982

ROGERS, G. J.; SAWYER, D. E.; JESCH, R. I.

Semiconductor Measurement Technology: Measurement of Transistor

scattering Parameters

NBS SP 400-5

January 1975

SANGSTER, R. C.

Relevancy of Measurements by a Systems Approach

Proc., 1972 Joint Meas. Conf., 21-23 June, 1972, Boulder, Co, pp. 31-37

1972

Trans. Matrix Description of the National system of Physical

Measurements

NBSIR 75-943

August 1976

Collected Executive Summaries: Studies of the National Measurement system $1972-75$

NBSIR 75-947

September 1976

study of the National Measurement system. Final summary Report, 1972-75 NBSIR 75-925

December 1976

Collected Executive Summaries: Studies of the National Measurement system

NBSIR 75-947-Rev. S.

February 1978

SCAVENNEC, A.; NARMAN, N. S.

A Simple Passively Mode-Locked CW Dye Laser

IEEE J. Quantum Electron., Vol. QE-10, pp. 95-96

January 1974 
SCOTT, W. W., Jr.

New Coaxial RF-DC Ammeter

IEEE Trans. Instrum. Meas., Vol. IM-19, No. 4, pp. 318-323 November 1970

Batteries Used with Law Enforcement Communications Equipment: Chargers and Charging Techniques

NBS Rept. 10732

June 1973

STOLTENBERG, R. E.

RF Null Detector NBS/SND

NBSIR 73-302

June 1973

Detection of Human Intruders by Low Frequency Sonic Interferometric Techniques

NBSIR 74-364

May 1974

STOLTENBERG, R. E.; WITTMANN, R. C.

Acoustical Interferometer Wader AIW Final Report of Advanced Development Test and Evaluation

NBSIR 82-167(R)

June 1982

TAGGART, H. E.; WORKMAN, J. L.; NELSON, R. E.

Emergency Locator Transmitters: Effective Radiated Power Levels and Techniques of Determining Effective Radiated Power

NBS Rept. 9769

February 1971

VANZURA, E. J.

Creating CSUBs written in FORTRAN That Run in BASIC

Proc., INTEREX Technical Computer Users Conf.,

7-12 August, 1988, Orlando, FL, paper 20, pp. 1-18 1988

Creating CSUBs in BASIC, Part I

HP Design and Automation Magazine, pp. 18-21

October 1988

Creating CsuBs in Basic, Part II

HP Design and Automation Magazine, p. 25 November 1988

WACKER, P. F.; CORD, M. S.; BURKHARD, D. G.; PETERSEN, J. D.; KUROL, R. F. Microwave Spectral Tables, Volume III, Polyatomic Molecules with

Internal Rotation

NBS MN 70, Vol 3.3, p. 276

June 1969

WAIT, D. F.; BEATTY, R. W.

The 1973 Intl. Microwave symp.

IEEE Trans. Microwave Theory Technol., Vol. MTT-21, No. 12, pp. 747-751

December 1973

WAIT, J. R.; HILL, D. A.

Fields of a Horizontal Loop of Arbitrary Shape Buried in a Two-Layer Earth

Radio Science, Vol 15, No. 5, pp. 903-912

September-October 1980 
WAREFIELD, J.P.

Addendum to Earth Terminal Measurement System Maintenance Manual NBSIR 81-1641

October 1981

WEIDMAN, M. P.; CAMPBEIL, E.

A Method for Designing Multi-Screw Waveguide Tuners

NBS Tech. Note 393

October 1970

WEITZEL, D. B.; LOWE, L. T.; ELLERBRUCB, D. A.; CRUZ, J. E.; SINDT, C. F. Hydrogen Slush Density Reference System

NBS Rept. 9796 June 1971

NASA-CR-124764

June 1974

WEARTON, M. J.; FRUSB, C. L.; NABMAN, N. S.

A Video Recorder for Coherent Doppler Radar

IEEE Trans. Geosci. Electron., Special Issue on Radio Metrology, $\mathrm{GE}-17$, No. 4, pp. 171-178

October 1979

WILDHACK, W. A.; MASON, H. L.; POWERS, R. S. Jr.

Accuracy Charts for RF Measurements

Proc., IEEE, Special Issue on Radio Meas. Methods and Standards,

Vol. 55, No. 6, pp. 1056-1063

June 1967

WYSS, J. C.; STWALLEY, W. C.; KOCH, M. E.; VERMA, K. K.

Laser Fluorescence Diagnostics of Lithium Vapor for Inertial Confinement Applications

Topical Meeting on Inertial Confinement Fusion, February 1980, San

Diego, CA 1980

YAGEJIAN, A. D.

Theory of Adjoint Reciprocity for Electroacoustic Transducers NBSIR 73-329 February 1974

Direct Integration of the Field Equations for Electroacoustic Transducers

IEEE Lett., Vol. 62, No. 6, pp. 858-859

June 1974

A Direct Approach to the Derivation of Electric Dyadic Green's Functions AP-Symposium Dig., October, 1976, pp. 76-156 October 1976

NBS Tech. Note 1000 January 1978

Electric Dyadic Green's Functions in the Source Region

Proc., IEEE, Vol. 68, No. 2

February 1980 
YAGHJIAN, A. D. (cont.)

Augmented Electric- and Magnetic-Field Integral Equations

Dig., Intl. Union of Radio Sci. (URSI) Symp. on EM Waves, Munich Germany

$$
\text { August } 1980
$$

Radio science, Vol. 16, No. 6; pp. 987-1001

November-December 1981

Reply to Criticism on Electric Dyadic Functions in the Source Region Proc., IEEE, Vol. 69, No. 2 February 1981

A Delta-Distribution Derivation of the Electric Field in the source Region

Dig., 1981 IEEE/AP-S Symp.

June 1981

YOUNG, M.; LAWTON, R. A.

Saturation of Optical Detectors at High Modulation Frequency

Proc., Annual Meeting Opt. Soc. of America, 12 October, 1977, Toronto, Canada, Vol. 67 , p. 1398 1977

ZAPF, T. L.; HARVEY, M. E.; LARSEN, N. T.; STOLTENBERG, R. E.

Ultrasonic Calorimeter for Beam Power Measurements from 1 to

15 Megahertz

NBS Tech. Note 686

September 1976

Proc., 1976 Ultrasonics Symp., 29 September-1 October, 1977, Annapolis, MD, IEEE Cat 76, CH1120-5SU, pp. 573-576 1977 
ADAIR, R. T. $\cdot \cdot \cdot \cdot \cdot \cdot \cdot \cdot \cdot \cdot 63,65,66,68$ ADAMS, J. W. $24,26,33,38,39,43,45,47,49,51,77,79$

AGEE, D. A.

AGY, D. L.

ALIEN, J. W.

ALLRED, C. M.

ALSPACH, W. J.

ANDERSON, I. R .

ANDERSON, $W . T$

ANDREWS, J. R.

ANSON, W. J.

ARTHUR, M. G.

BABJI, $T$.

BACHMAN, D. D.

BAILEY, R. A.

BAIRD, R. C.

BARER-JARVIS, J.R.

BALDWIN, E. E.

BEAN, J. L.

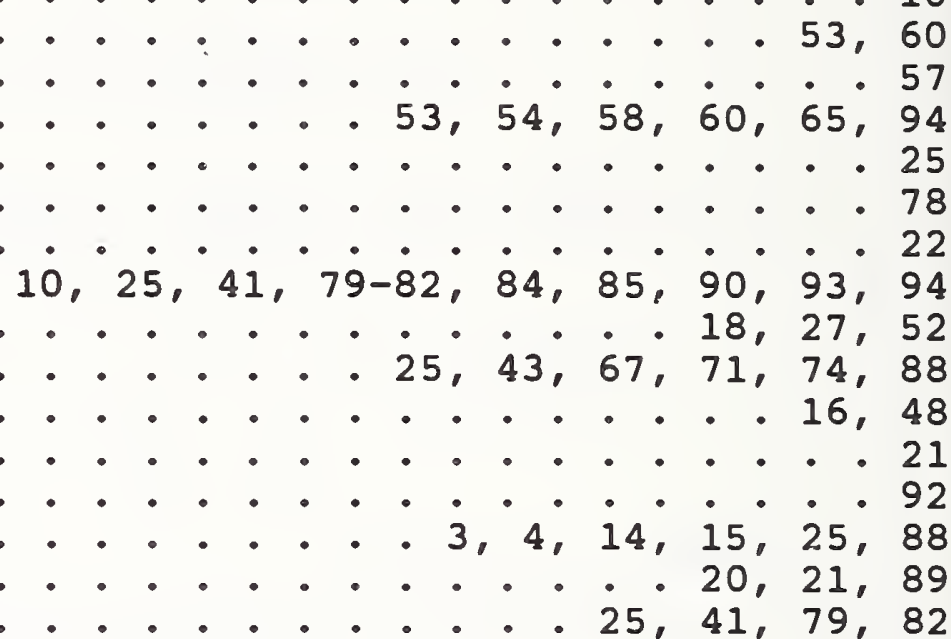

BEATTY, R. W.

BELL, B. A.

BELSHER, D. R.

. . . 29

BENNETT, W. S.

BENSEMA, W. D.

BEREUTER, W. A.

BERRY, I. S.

BERRY, L. A.

BIGELOW, J. M.

BLACKBURN, D.

BLANK, $W$.

BORSERO, $M$.

BOUILLE, R.

BOWMAN, R. R.

BOYLE, D. R.

BOYNE, H. S.

.. . 82

• • • . $25,39,76,77$

$3,24-26,39,40,43,47,88$

BRANDENBERGER, H.

............ . . 76

BREMER, T. H.

BUCHHOLZ, F. IM.

BUDLONG, A.

BURKHARD, D. G.

BURNS, J. G.

BUSSEY, H. E.

CAMELL, D. G.

CAMPBELL, E.

CANALES, $S$.

CAVCEY, $\mathrm{K}$. $\mathrm{H}$.

CHANG, A. T. C.

CHANG, D. C.

CHESNUT, S. M.

CHEW, H.

CLAGUE, F. R.

CLARK, H. E.

COLLIER, R. S.

DAYWITT, W. C.

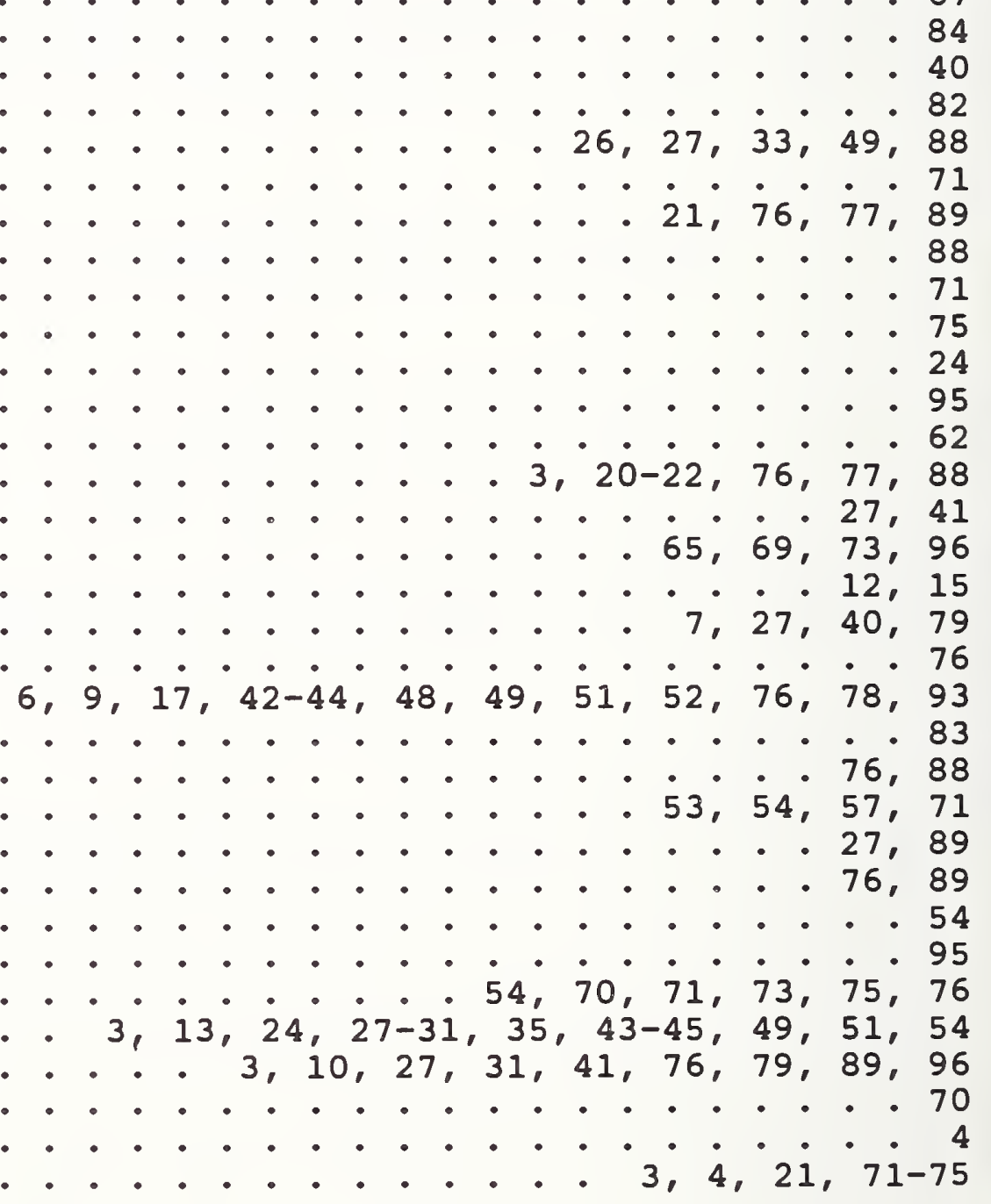


KOEPKE, G. H.

$7,22,26,29,30,35,40,41,43$

KOMAREK, E. L.

KREMER， D. P.

KUFFEL, J.

KUKOL, R. F.

LADBURY, J. M. $6,9,10,12,14,15$

LARSEN, E. B.

LARSEN, $N$. T.

LARSON, T. R.

LARSON, W.

LAWTON, R. A.

LEWIS, R. L.

$0 . \cdot \cdot \cdot \cdot \cdot \cdot 65,68,70$

LITTLE, W. E.

$10,22,40,41,65,79,80,82,84,85,87,92-94,97$

LIU, B. H.

LIVINGSTON, E. M.

$21,22,65,93$

LLOYD, F.

LOWE, L. T.

LOWELL, W. R.

LUFT, P. E.

LYONS, R. M.

MA, M. T.

MACREYNOLDS, $K$.

MAJOR, J. R.

MAJUMDAR, A.

MALEWSKI, R.

$53,65,66$

MANNEY, C. H.

MARKS, R. B.

MARLER, F. E.

MARTZLOFF, F. D.

MASCLET, G.

MASON, H. L.

MASTERSON, K. D.

MAY, W. G.

MCCAA, W. D. Jr.

MCFARLANE, E. J.

ig 90

89,96

$3,10,22,27,31,40,41,43-45,88$

MCLAUGHLIN, R. G.

MEDLEY, H. W.

MELQUIST, D. G.

METZKER, R. F.

- 33

MEYER, $K$.

MIERS, T.

MILLER, C. K. S .

MONTGOMERY, J. P.

76

$11,17,31,40,42-44,48-51$

MORGAN, W. L. .

66,69

.87

40,92

53,93

MORRIS, D.

$57,58,66,67,70$

MUTH, L. A.

NAHMAN, N. S.

NELSON, R. E.

NEWELL, A. C.

NORGARD， J. D.

ONDREJKA, A. R.

ORR, R. D.

PAGE, J. M.

PAULTER, N. G., Jr.

PEISER, H. S. . .

PERERA，S.

PERSINGER, R. R.

PETERSEN, J. D.

PETERSON, R.

PHILLIPS, K. R.

$25,44,45,47,67,74,75,89, \dot{9}, 93$

POWERS, R. S. Jr.

78,93

RANDA, J. P.

REBULDELA, G.

REEVE, G. R.

REIDY, A. R.

REPJAR, A. G.

RIAD, S. M.

$39,4 \dot{0}, 4 \dot{5}, 6 \dot{6}, 8 \dot{0}, 82-87,94,96$

...... 3, $5,9-15,17,45$

$10,18,35,41,45,51,52,61,67,69,79,80,84,86,87$

$9,18,22,25,39,40,46,52$

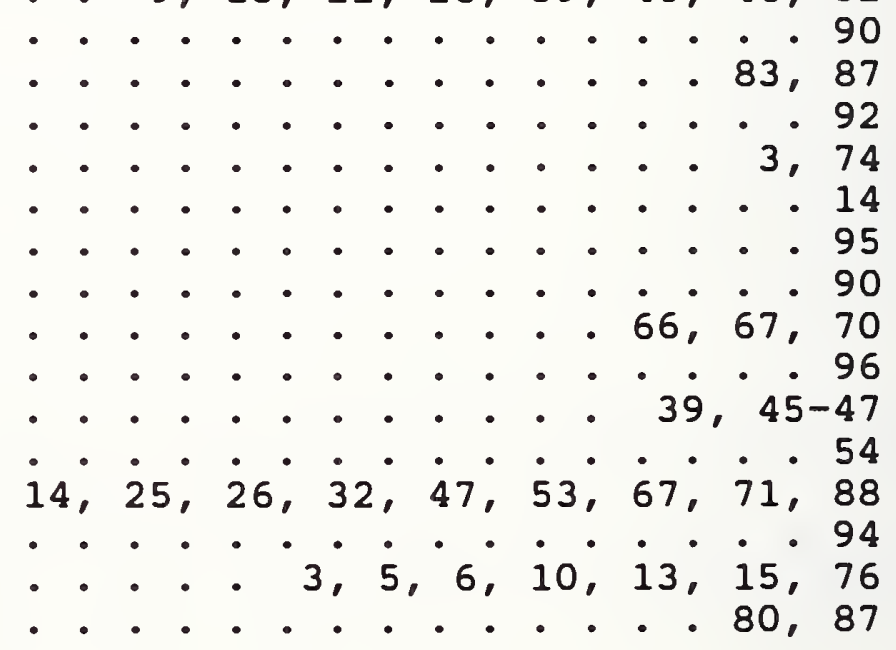


RICHMOND, J. H.

SEGA, R. M.

IAGGART, H. E.

$16,20,24,48,95$

TAMURA, D. T

TAUSCH, $\mathrm{H} . \mathrm{J}$. 


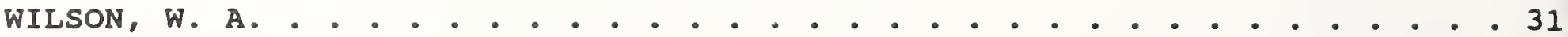

WITTMANN, R. C. . . . . . . . . . . . . . 6, 11, 17-19,78,94,95 WOODRUFF, J. J. • . . . . . . . . . . . . . . . . . . . . . . . . . 4 WORKMAN, J . L. . . . . . . . . . . . . . . . . . . 16, 30, 31, 95 WU, D. I. . . . . . . . . . . . . . . . . . . . . . 24, $35,51,52$ WU, Y. . . . . . . . . . . . . . . . . . . . . . . . . . . . . . 24 WYSS, J. C. • . . . . . . . . . . . . . . . . . . . 18, 39, 52, 96 YAGHJIAN, A. D. . . . . . . . . . . . . 6, 14, 16, 18, 19, 77, 96, 97 YATES, B. C. • . . . . . . . . . . . . . . . . . 54, 65, 68, 70 YODOSHIMA, I. . . . . . . . . . . . . . . . . . . . . . . . . 40

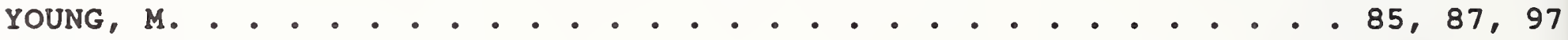
ZALTSMAN, E. B. • . . . . . . . . . . . . . . . . . . . . . . . . 21 ZAPF, T. L. . . . . . . . . . . . . . . . . . . . . . . 65, 97 
BL-114A

$(5-\infty)$
U.S. DEPARTMENT OF COMMERCE NATIONAL INSTITUTE OF STANDARDS AND TECHNOLOGY

BIBLIOGRAPHIC DATA SHEET
1. PUBLCATION OR REPORT NUMBER NISTIR 3993

2. PERFORMING ORGAMIZATION AEPOAT NUII B92-0236

3. PUBLCATION DATE

A. TMTUE AND SUETITLS

A Bibliography of the NIST Electromagnetic Fields Division Publications

5. AUTHOR(S)

Ruth Marie Lyons; Kathryn A. Gibson (Editors)

6. PERFORMING ORQANIZATION (IF JOINT OR OTHER THAM MIST, SEE INSTRUCTIONS)

U.S. DEPARTINENT OF COMLERCE

MATIONAL INSTITUTE OF STANDARDS AND TECHMOLOQY

BOULDER, COLORADO $80303-3323$

7. CONTRACT/GRANT NUMBER

8. TYPE OF REPOAT AND PEAIOD COVERED

9. SPONSOAIMG ORCAMIZATION MAME AND COMPLETE ADDRESS (STREET, CTT, STATE, ZIP)

10. SUPPLEMENTAAY MOTES

Supersedes NISTIR 3973

11. ABSTRACT (A 200-WOAD OALESS FACTUAL SUMMAAY OF MOST SIQMIFICANT IMFOAMATION. IF DOCUMENT INCLUDES A SIGMIFICANT BIBLOQRAPHY UTERATURE SUAVEY, MENTION IT HERE.)

This bibliography lists the publications by the staff of the Electromagnetic Fields Division of the National Institute of Standards and Technology for the period January 197 through July 1992. It supersedes NISTIR 3973 which listed the publications of the Electromagnetic Fields Division from January 1970 through July 1991. Selected earlier publications from the Division's predecessor organizations are included.

12. KEY WOADS (6 TO 12 EMTRIES; ALPMABETICAL OADEA; CAPTTALIZ ONLY PAOPER MAMES; AND SEPARATE KEY WOADS BY SEMICOLONS)

antennas; dielectric measurements; electromagnetic interference; microwave metrology; mic wave power; impedance; attenuation; near-field antenna measurements; noise; non-ionizing radiation; radiation hazards; standards; time domain; waveform metrology

\section{AVALABIUTY}

\begin{tabular}{|l|}
\hline$X$ \\
\hline \\
\hline \\
\hline$X$ \\
\hline
\end{tabular}

UMLUATED

FOR OFFICIAL DISTAIBUTION. DO NOT RELEASE TO MATIOMAL TECHMICAL INFORMATION SERVICE (NTIS).

ORDER FROM SUPERINTENDENT OF DOCUMENTS, U.S. GOVERMMENT PRINTING OFFICE, WASHINGTON, DC 20402.

ORDER FROM MATIOMAL TECHNICAL INFORMATION SEAVCE (MTIS), SPRINGFEL, VA 22161.
14. NUMBER OF PAINTED PAGES 105

15. PAICE 
